\title{
PSYCHOPHYSICS AND PHYSIOLOGY
}

\section{OF ATTENTIONAL INFLUENCES ON VISUAL \\ MOTION PROCESSING}

\author{
PhD Thesis \\ in partial fulfilment of the requirements \\ for the degree "Doctor of Philosophy (PhD) / Dr. rer. nat." \\ in the Neuroscience Program \\ at the Georg August University Göttingen, \\ Faculty of Biology \\ submitted by \\ Katharina Anton-Erxleben \\ M. Sc. (Neurosciences) \\ born in \\ Stanford (USA)
}

Göttingen, March 2008 
Herewith I declare that I have written this thesis independently and with no other aids and sources than quoted.

(Katharina Anton-Erxleben) 


\section{Acknowledgements}

The work presented in this thesis would never have been possible without support. First of all, I would like to thank Prof. Stefan Treue for giving me the opportunity to work in his laboratory. Throughout the years, I have enormously benefited from his excellent supervision and commitment. I also would like to thank Dr. Alexander Gail, Prof. Jens Frahm and Prof. Thomas Rammsayer for being part of my thesis committee and supporting me with expert advice.

I am especially grateful to Dirk Prüsse, Leonore Burchardt, Sina Plümer and Kathrin Grell for invaluable help with taking care of the monkeys and providing solutions for all sorts of technical problems. Also, I want to thank Florian Pieper for frequent help with the electrophysiological procedures.

I want to say thank you to Thilo Womelsdorf, who introduced me to the fascination of receptive field mapping and gave me the opportunity to contribute to one of his studies. I also want to thank Christian Henrich, who put a lot of effort and motivation into help with the psychophysical part of this thesis. A special thanks goes to Valeska Stephan for all the long days and weeks of recording data together, and for making this demanding phase of the PhD work a lot more fun. I am also extremely grateful to Tzvetomir Tzvetanov for critical and productive discussions and invaluable help with data analysis and statistics.

I would like to thank Sabine Stuber and Beatrix Glaser for their help with all the administrative stuff, and Ralf Brockhausen, not only for the support in computerrelated issues and making last-minute poster prints possible, but also for being a great office mate.

I would like to thank Pinar Boyraz for making the conference trips to Atlanta and San Diego a fun experience, and I would like to thank her as well as the other members of the cognitive neuroscience lab for making the work atmosphere so enjoyable. 
Last but not least I want to thank my family and friends - Friederike, Rainer, MarieLuise, Monika and Tina - for their encouragement and support, but also for helping me to keep a healthy balance between work and life.

And thank you for everything, Christoph. 


\section{Contents}

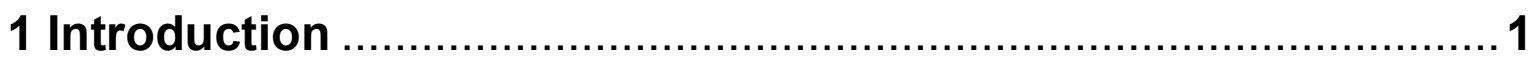

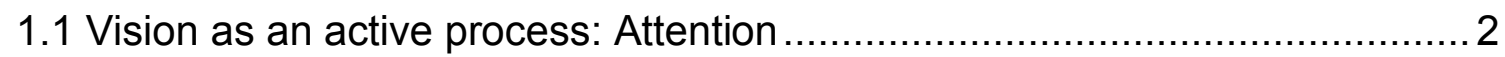

1.1.1 Attentional selection.......................................................... 3

1.1.2 Physiological mechanisms of attention ....................................... 4

1.1.3 Psychophysical mechanisms of attention ...................................... 7

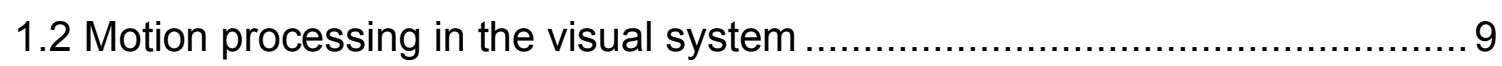

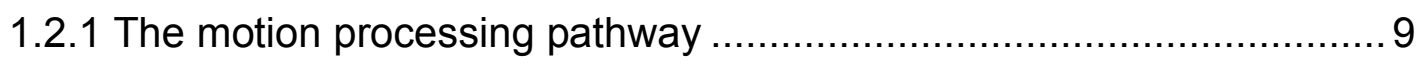

1.2.2 Response properties of MT receptive fields and their role in motion processing.................................................................... 11

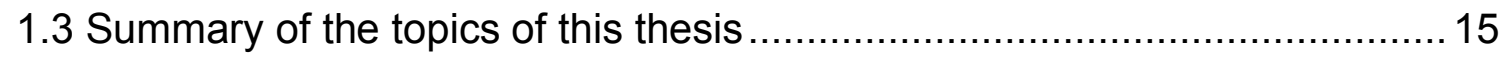

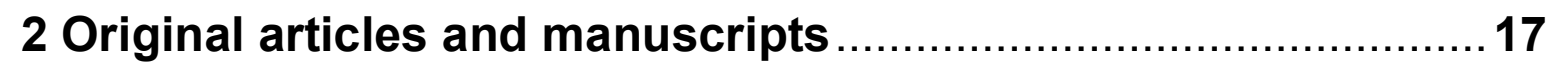

2.1 Dynamic shifts of visual receptive fields in cortical area MT by

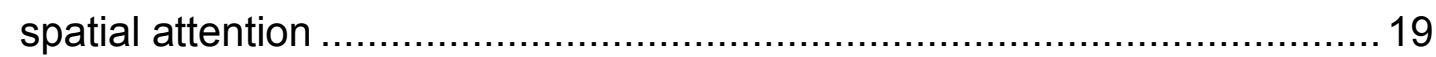

2.2 Attention modulates center-surround structure in area MT ...................... 33

2.3 Receptive field shift and shrinkage in macaque area MT through

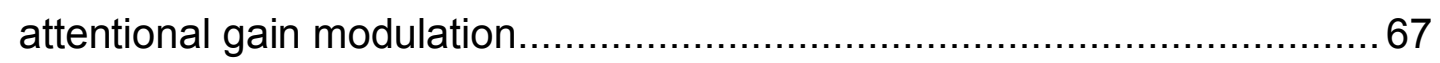

2.4 Attention changes perceived size of moving visual patterns ..................... 95

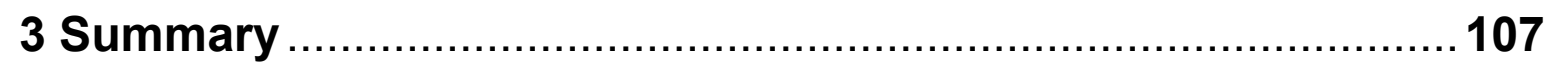

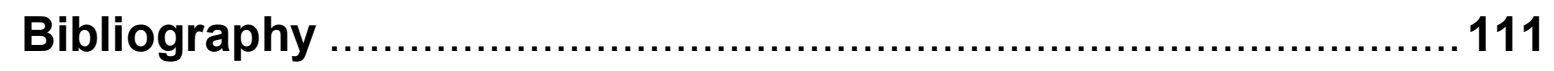

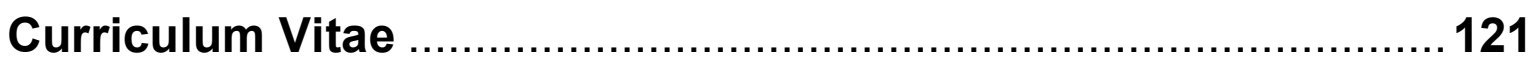





\section{Introduction}

Perception is not an objective representation of the sensory input, but is influenced by the behavioral state of the organism. Attention is one of the factors that biases perception by the modulation of sensory processing. This thesis deals with attentional effects on the processing of sensory information in the visual system. Specifically, the processing of visual motion serves as a model system to study how attention shapes the spatial selectivity of single neurons and how this change in spatial tuning might influence the perception of spatial relations. The thesis' main part consists of two related studies in which single-cell recordings from the medial temporal area (MT) of awake behaving macaques were used to investigate attentional modulation of receptive field profiles (spatial selectivity profiles), one computational study in which these effects were modeled, and one psychophysical experiment with human subjects in which predictions from the physiological results were tested.

This chapter will give a general introduction to attentional influences on visual processing at the physiological and the behavioral level, to the motion processing pathway of the primate visual system, and to the properties of receptive fields in area MT. The second chapter will contain the research articles and prepared manuscripts; and the third chapter will give a summary and general discussion of the findings presented in this thesis. 


\subsection{Vision as an active process: Attention}

Visual perception is not an objective reproduction of the visual scene. Instead, different filter mechanisms serve to transform the visual image into a sparse representation of only the most relevant aspects. Some of these filters are 'hardwired' into the processing machinery and select information according to computational relevance; others are variable and can be adapted to situational demands. Attention is an example for an adaptive filter: it is the most important mechanism to filter information according to behavioral relevance.

Visual neurons often respond best to a stimulus that is relatively small and covers only a certain location in the visual field, the neuron's classical receptive field. If the same stimulus extends beyond the classical receptive field, into the surround, the neuronal response is often suppressed. Such an antagonistic center-surround receptive field structure emphasizes discontinuities in the visual field. With each increasing level of the visual processing hierarchy, there is a convergence of input into fewer and larger receptive fields, which are selective for increasingly complex stimuli, while redundant information is discarded. This results at the higher stages of the visual system in an almost exclusive representation of the information which is most relevant computationally for an efficient description of the visual image (see Lennie, 1998; Lennie, 2003, for reviews).

Furthermore, the central part of the visual field is overrepresented throughout the visual hierarchy: already in the retina, the mapping of photoreceptors to retinal ganglion cells has a finer resolution near the fovea than in the periphery, and in many visual areas, a greater proportion of cortex is devoted to processing input from the central visual field than from the periphery (cortical magnification). Signals from these locations are processed with greater accuracy than others.

If we are interested in a particular location in the periphery, we can make use of the enhanced processing in the central visual field if we make an eye movement and overtly focus the location of interest. We are also able, however, to covertly attend to this location, without moving the eye, and thereby improve processing of 
signals from this location. The following sections will give an introduction to attentional selection and its mechanisms on the physiological as well as the behavioral level.

\subsubsection{Attentional selection}

Attentional selection can target spatial locations (like a 'spotlight'), but also stimulus features or whole objects. Spatial attention has been demonstrated in psychophysical as well as physiological experiments: Behaviorally, information from an attended location is processed faster and more accurately (e.g. Posner, 1980); physiologically, spatial attention modulates responses of those neurons whose receptive fields overlap the attentional focus (Tootell et al., 1998; Brefczynski and DeYoe, 1999; Müller et al., 2003). Feature-based attention, on the other hand, has been shown to modulate responses of those neurons that are involved in processing the attended feature, for example direction of motion, independent of the spatial location of their receptive fields (Treue and MartinezTrujillo, 1999; Sàenz et al., 2002; Martinez-Trujillo and Treue, 2004). Behaviorally, attentional selection of features facilitates processing of spatially distant stimuli sharing the attended feature (Sàenz et al., 2003), and might improve performance in visual search tasks in which a specific stimulus feature has to be discriminated among distractor stimuli (Mounts and Melara, 1999). Object-based attentional selection has been demonstrated by the spread of behavioral benefits as well as physiological correlates of spatial attention to other locations of the same object, even if they are behaviorally irrelevant, but not to equidistant locations that do not belong to the same object (Roelfsema et al., 1998; Müller and Kleinschmidt, 2003). Other studies have shown that also directing attention to a feature of an object can enhance the neuronal response to other, behaviorally irrelevant, features of the same object (Wojciulik et al., 1998; O'Craven et al., 1999; Serences et al., 2004).

The allocation of attention can be guided voluntarily by top-down processes, but can also be captured automatically by exogenous cues like abrupt stimulus onsets; this bottom-up type of attention is typically effective within a time window 
of 70-150 ms after the cue onset, while voluntary attention is slower and needs at least $150 \mathrm{~ms}$ to become effective in a psychophysical task (Nakayama and Mackeben, 1989; Liu et al., 2007).

\subsubsection{Physiological mechanisms of attention}

\section{Multiplicative modulation}

Early physiological studies have demonstrated attentional influences on visual processing by recording from single neurons while a monkey switched attention between two stimuli, one inside and one outside the neuron's receptive field. Allocating attention to the stimulus inside the receptive field generally enhanced the neuron's response. Neurons in many visual areas have a preference for a stimulus feature like a specific orientation or direction of motion (they are tuned for the feature; see below), and early studies have suggested that attention sharpens the neurons' selectivity (Haenny and Schiller, 1988; Spitzer et al., 1988). More recent research however shows that attention multiplicatively scales responses without changing the selectivity of single neurons, that is without changing the width of their tuning curves (McAdams and Maunsell, 1999; Treue and MartinezTrujillo, 1999). In the case of orientation tuning (McAdams and Maunsell, 1999), the effects of attention were explained with a response gain model, meaning that responses above the spontaneous level were scaled by a fixed factor. In the case of direction tuning (Treue and Martinez-Trujillo, 1999), a response gain model as well as an activity gain model, which assumes a multiplicative scaling of all firing rates, could account for the data. Studies measuring the attentional effects on stimuli of different contrast have come to a slightly different conclusion: they proposed a contrast gain model in which attention shifts the contrast response function towards lower contrast, effectively increasing the contrast of the attended stimulus (Reynolds et al., 2000; Martinez-Trujillo and Treue, 2002). A recent study has directly compared contrast gain, response gain, and activity gain models and found that all provide extremely good accounts of the observed effects of attention, but response and activity gain models were slightly superior than the contrast gain model, consistent with a multiplicative modulation of responses to all stimuli, including variations of contrast (Williford and Maunsell, 2004). 
Multiplicative modulation by 'gain fields' has been observed in many different processing contexts and may be a common principle underlying various coordinate transformations, for example the transformation from eye-centered into body-centered coordinates which is important for reaching movements (see Salinas and Thier, 2000, for a review). Similarly, attentional gain fields can be used to transform an eye-centered into an object-centered or attention-centered reference frame; such a transformation would enable scale- and position-invariant recognition of objects (Salinas and Abbott, 1997; see also Olshausen et al., 1993). Support for attentional gain fields comes from the observation that receptive fields in V4 are scaled with different positions of the attentional focus (Connor et al., 1996, 1997).

\section{Biased competition and feature-similarity gain modulation}

In natural situations, the receptive field of an extrastriate neuron usually overlaps with more than one stimulus. If two stimuli fall into a neuron's receptive field, one preferred and one anti-preferred, the response is typically an average of the responses to each of the stimuli presented alone. Switching attention between the two stimuli in such a setting biases this average towards the response that would be obtained by presenting the attended stimulus alone, so attention effectively reduces the influence of the unattended stimulus (Moran and Desimone, 1985; Reynolds et al., 1999; Treue and Maunsell, 1996; Treue and Maunsell, 1999). The biased competion model of attention states that in such situations, multiple stimuli compete for neuronal representation and attention biases the competition in favour of the attended stimulus (Reynolds et al., 1999). It has been suggested that the mechanism by which attention reduces the ability of the unattended stimulus to drive the cell's response is a contraction of receptive fields around the attended stimulus (Moran and Desimone, 1985). The feature-similarity gain model provides another interpretation of these data: according to this model, the response of a neuron is multiplicatively scaled by a factor depending on the match between the features of the attended stimulus and the preferred features of the neuron, independent of its receptive field location and independent of the stimulus currently inside the neuron's receptive field (Treue and Martinez-Trujillo, 1999). This model can account for the differential modulation of the response to two 
stimuli, and also for the effects of feature-based attention found with one stimulus inside the receptive field (Martinez-Trujillo and Treue, 2004).

\section{Representation of attentionally selected information}

Attention does not only modulate firing rates, but also synchronizes firing in neuronal populations that are involved in representing the attended stimulus, thereby increasing synaptic transmission efficacy within these populations (Steinmetz et al., 2000; Fries et al., 2001). This synchrony might be induced by volleys of synchronous action potentials along descending feedback pathways and then play a mechanistic role in (top-down) attentional selection (see Niebur et al., 2002, for a review) and the formation of a 'perceptual coherence field', an ensemble of neurons which represent an object by their coordinated activity (see Serences and Yantis, 2006, for a review).

Attention has been shown to affect neuronal activity already at the earliest stages of the visual processing hierarchy, the lateral geniculate nucleus (LGN) and the primary visual cortex (V1), but there is a tendency for attentional effects to increase in magnitude with the level of hierarchy (e.g. O'Connor et al., 2002; Sàenz et al., 2002). So from a sensory representation of the environment at earlier stages of the visual system, there is a gradual transformation towards a representation of perceptual interpretation and behavioral state (see Treue, 2003a, for a review). Visual processing can be viewed as a 'multi-stage selection process' in which relevant information is more and more favoured at the expense of irrelevant information. Hard-wired filters, bottom-up and top-down attentional mechanisms interact to create a saliency map of the visual scene, which encodes regions of interest, and could serve to guide eye movements towards these locations (see Treue, 2003b; Serences and Yantis, 2006, for reviews). The premotor theory of attention states that the selection of eye movement goals and attentional selection indeed have a common mechanism (Rizzolatti et al., 1987); supported by the observation that microstimulation of the frontal eye field (FEF) below the threshold needed to elicit eye movements induces attention-like gain changes in simultaneously recorded V4 neurons (Moore and Armstrong, 2003). 


\subsubsection{Psychophysical mechanisms of attention}

Selective attention enhances behavioral performance in a variety of psychophysical tasks: attention improves accuracy in detecting and identifying stimuli, in discriminating stimulus features, in spatial resolution and visual search tasks; and speeds up processing of attended stimuli (Posner, 1980; Nakayama and Mackeben, 1989; Hawkins et al., 1990; Yeshurun and Carrasco, 1998; Mounts and Melara, 1999; Yeshurun and Carrasco, 1999; Dobkins and Bosworth, 2001; Sàenz et al., 2003; Liu et al., 2007).

An early theory of attention, the feature integration theory, is based on the observation that in visual search, targets that differ by one feature from the distractors 'pop out' and are found easily independent of the number of distractors, while targets that are defined by a conjunction of features need more time with each distractor added (the set-size effect). This has led to the conclusion that there are two processing stages: one preattentive, parallel search that identifies single features, and a second, serial search that requires focused attention, which is of limited capacity, to scan each item and decide if it is the target or not (Treisman and Gelade, 1980). According to this view, attention is necessary to bind features together in a saliency map. More recent research has challenged the feature-integration theory: first, attention has been shown to act on targets defined by a single feature, showing that processing of this kind of stimuli is not preattentive (Joseph et al., 1997), and second, it has been pointed out that the setsize effect could be explained assuming neither serial processing nor a limitedcapacity mechanism. Instead, all items could be scanned in parallel, but noise would degrade the signal with increasing number of distractors (e.g. Eckstein, 1998). Others have suggested that for purely statistical reasons, the likelihood of confusing the target with a distractor increases at the decision level with the number of distractors (e.g. Dobkins and Bosworth, 2001).

A related question has been by what mechanism attention exerts the improvements in performance that are observed in visual search and other tasks when attention is directed to a particular location. Several studies have suggested that attention reduces external noise, which can be for example noise in the signal, 
noise from neighboring distractors, or uncertainty about the attended position (e.g. Dosher and Lu, 2000; Dobkins and Bosworth, 2001), while others have claimed that attention influences the decision process without changing the perceptual quality of the attended stimulus itself, by weighting the signal from the attended location stronger than that from the unattended location (e.g. Eckstein et al., 2002). Some recent studies, however, provided strong evidence for an attentional mechanism that enhances the signal: most of them are based on attentional effects on suprathreshold targets in noiseless displays, ruling out the reduction of external noise (Carrasco et al., 2000; Carrasco et al., 2002). These findings do not rule out the reduction of internal noise as an attentional mechanism.

Perhaps the strongest claim for an enhancement of the perceptual quality of the stimulus comes from a series of experiments demonstrating that attention changes the phenomenal appearance of several stimulus attributes. For example, attended stimuli appear as stronger in contrast than unattended stimuli (Carrasco et al., 2004), which is interpreted as the behavioral correlate of the shift in the contrast response function observed in physiological experiments (see above). Besides apparent contrast, attention has been shown to modulate perceived spatial frequency, gap size, motion coherence, color saturation, flicker rate, and speed (Gobell and Carrasco, 2005; Liu et al., 2006; Fuller and Carrasco, 2006; Montagna and Carrasco, 2006; Turatto et al., 2007). 


\subsection{Motion processing in the visual system}

\subsubsection{The motion processing pathway}

Analysis of different features in the visual scene occurs largely in parallel within specialized pathways of the mammalian visual system (see e.g. Ungerleider and Mishkin, 1982; Goodale and Milner, 1992; for reviews). Of these features, motion is one of the most important: without accurate perception of speed and direction we cannot estimate where a moving object, for example a car, will be in a moment, and our interaction with the environment is severely impaired (Zihl et al., 1983). Motion enables us to discriminate moving objects from stationary background, contributes to the perception of self-motion in the environment (Regan, 1986; Warren and Hannon, 1988), and provides information about the three-dimensional structure of objects (e.g. Treue et al., 1991).

Motion processing is localized to areas of the dorsal visual pathway, which is dominated by input from retinal $M$ cells and magnocellular layers of the LGN (Maunsell et al., 1990; Yabuta, 2001), and projects via areas V1, V2, V3, MT, the medial superior temporal area (MST), and the fundal superior temporal area (FST) to posterior parietal cortex (Baizer et al., 1991). The segregation from the ventral pathway is not absolute: there is some amount of input from parvo- and koniocellular layers to the dorsal pathway (Sincich et al., 2004; Nassi and Callaway, 2006), and connections to areas involved in the processing of color and form exist (e.g. V4; Ungerleider and Desimone, 1986; Baizer et al., 1991).

\section{Motion analysis starts in striate cortex}

Area V1 is the earliest stage in the visual processing hierarchy where directionally selective neurons are found (e.g. Hubel and Wiesel, 1977) that respond strongly to stimuli moving in a particular direction (the preferred direction), weakly to the opposite direction (the anti-preferred direction), and intermediate to directions in between. Such direction tuning can be typically well approximated by a Gaussianshaped tuning curve. $\mathrm{V} 1$ also contains neurons that are tuned for the speed of a moving stimulus (Orban et al., 1986). A specialized subset of neurons that are 
highly directionally selective and sensitive for low contrast are concentrated in layer 4B and project either directly or via thick stripes of V2 to area MT, which lies in the posterior bank of the superior temporal sulcus (Maunsell and Van Essen, 1983a; Movshon and Newsome, 1996; Sincich and Horton, 2003; Nassi and Callaway, 2006).

\section{Area MT is a critical stage within the motion processing pathway}

In area MT, which is the homologue of human V5, the majority of neurons is directionally selective (Dubner and Zeki, 1971; Zeki, 1974; Albright, Desimone, Gross, 1984). Almost all neurons also have a disparity tuning, and many are tuned for stimulus speed (Maunsell and Van Essen, 1983b). Directionally selective neurons are organized in columns (Maunsell and Van Essen, 1983b; Zeki, 1974; Albright, Desimone, Gross, 1984), which overlap with disparity columns (DeAngelis and Newsome, 1999). Direction columns of smoothly varying preferred directions are thought to run along columns which have locally opposite preferred directions (Albright, Desimone, Gross, 1984). Neurons with similar preferred speeds are also arranged in clusters, but strict columnar organization has not been found (Maunsell and Van Essen, 1983b; Liu and Newsome, 2003).

Evidence that area MT is critical for the perception of motion comes from three types of experiments. First, lesions of area MT lead to severe and selective deficits in perceiving visual motion. These deficits can be overcome by training up to a large extent, which means that other pathways must be able to take over the lost functions, but a part of the impairment is permanent and cannot be recovered from (Newsome and Paré, 1988). Second, the behavioral choice in a motion discrimination task is correlated to neuronal responses in area MT (Britten et al., 1996); and third, electrical stimulation of clusters of directionally selective MT neurons biases decisions in such a task towards the preferred direction (Salzmann et al., 1990).

\section{Higher areas analyze complex motion}

Area MT sends output to areas involved in the analysis of more complex motion like area FST, MST and the ventral intraparietal area (VIP), and to areas that are associated with the generation of eye movements like the FEF and the superior 
colliculus (Maunsell and Van Essen, 1983a; Ungerleider and Desimone, 1986). Area FST contains a rather small proportion of directionally selective neurons; some of them respond preferentially to optic flow stimuli (Desimone and Ungerleider, 1986). Area MST contains a high proportion of neurons that are tuned to optic flow stimuli like rotation, expansion, contraction or spiral motion (Graziano et al., 1994). Responses to speed gradients found in area MT might provide the critical input from which these response properties in MST are built (Treue and Andersen, 1996; Martinez-Trujillo et al., 2005). Regions responsive to wide-field motion in MT (that is columns without antagonistic surround suppression, see below) project to the dorsal part of FST and to the ventral part of MST, while local motion regions (receptive fields with antagonistic surrounds) project predominantly to the dorsal part of MST (tested only in the owl monkey; Berezovskii and Born, 2000). This may be an indication for a parallelization of the analysis of self-motion in the environment, which is related to wide-field motion stimuli, and object motion, which relies on local motion stimuli. Area VIP contains a large number of neurons which are selective for the direction and speed of translational motion and distance in depth, similar to MT neurons. Some VIP neurons, however, respond selectively to stimuli moving in depth towards or away from specific parts of the face, independent of the stimulus' trajectory (Colby et al., 1993).

\subsubsection{Response properties of MT receptive fields and their role in motion processing}

Area MT contains a full representation of the contralateral visual field. This representation is biased, though, towards the lower visual field, which is overrepresented, and towards the central visual field (cortical magnification; Maunsell and Van Essen, 1987). Receptive field size increases with eccentricity; generally receptive fields are about 10 times larger than in V1 and are arranged in a rather crude retinotopic map (e.g. Maunsell and Van Essen, 1987).

\section{Area MT receptive fields are built from V1 input}

As mentioned above, receptive fields in area MT are selective for speed, direction, and disparity. There is evidence that these basic tuning properties of MT neurons 
are largely inherited from V1 input (see Born and Bradley, 2005, for a review). Because receptive fields in area MT are substantially larger than V1 receptive fields, it has been thought that MT integrates motion over a broader spatial range than $\mathrm{V} 1$, but recent studies have shown that MT neurons in fact compute stimulus motion over a similarly short spatial scale as V1 neurons (Livingstone et al., 2001; Churchland et al., 2005; Pack et al., 2006), and the characteristics of V1 inputs are discernable in the substructure of MT receptive fields (Livingstone et al., 2001; Pack et al., 2006).

\section{MT receptive fields process local and global motion}

MT however performs additional functions in integration and segmentation. While the small receptive fields in V1 are confronted with the aperture problem - moving edges seen through small apertures give ambiguous direction information -, MT contains neurons that are able to solve the aperture-problem: When stimulated with a plaid stimulus, which has two grating components each giving ambiguous direction information, these neurons respond to the (unambiguous) global motion of the stimulus, while $\mathrm{V} 1$ cells only respond to the motion of the components (Movshon et al., 1985; Rust et al. 2006). Also, MT neurons employ additional mechanisms to reduce noise in the motion signal: unlike V1 neurons, MT neurons' responses to a preferred stimulus are suppressed by simultaneous motion in the opposite direction (motion opponency; Snowden et al., 1991). Both are examples for context influences from within the classical receptive field.

As in many other visual areas, responses in area MT are also strongly influenced by contextual information from outside the classical receptive field. These context influences are mediated by the center-surround structure of many MT receptive fields. Surround effects in area MT have been shown to act over a large spatial scale, ranging from 3-4 (Raiguel et al., 1995) to 7-10 (Allman et al., 1985; Tanaka et al., 1986) times the radius of the classical receptive field, and are typically antagonistic (Allman, 1985; Tanaka et al., 1986; Bradley and Andersen, 1998; Lui et al., 2007), so that neurons with a surround respond better to local motion confined to the receptive field center while neurons without a surround respond well to global or wide-field motion. The exact proportion of surround neurons in area MT varies from $50 \%$ (Perge et al., 2005) to 79\% (Raiguel et al., 1995; Lui et 
al., 2007) in different measurements. Local motion selective neurons and global motion selective neurons are arranged in columns or patches (Born \& Tootell, 1992; Raiguel et al., 1995). The antagonistic tuning of MT center-surround structure favours segmentation of a moving stimulus from its background: responses are maximally suppressed when surround stimuli have the same direction, speed, and / or disparity as the central stimulus (Allman, 1985; Tanaka et al., 1986; Bradley and Andersen, 1998; Lui et al., 2007). This enables MT receptive fields to act as detectors of image discontinuities and makes MT a likely candidate for segmenting the visual image based on motion (Bradley and Andersen, 1998). The idea that MT uses motion to segment an object from its background receives further evidence from a study that shows differential effects of microstimulating local-motion and global-motion sites on eye movements: stimulating local-motion sites biases pursuit and saccadic eye movements towards the preferred direction, while stimulating global-motion sites biases eye movements towards the anti-preferred direction, consistent with simulating background motion which induces eye movements in the opposite direction (Born et al., 2000).

Nearly half of MT surrounds have been suggested to be asymmetrically concentrated on one side of the classical receptive field rather than being arranged circularly around it (Xiao et al., 1995), and asymmetric surrounds tuned for different speeds than the receptive field center are thought to mediate the ability to perceive 3D structure from motion (Buracas \& Albright, 1996; Liu and Van Hulle, 1998). It has been found that even classical receptive fields themselves in area MT are often tuned for specific speed gradients and respond better to such gradients than to flat speed profiles (Treue and Andersen, 1996; Martinez-Trujillo et al., 2005). Responses to speed gradients might contribute to the perception of 3D structure from motion.

Recent research has shown that in area MT, as well as in area V1, surround effects are not fixed for a given cell; instead, stimulus properties, perceptual context and attention can modulate the suppressive or integrative nature of influences from receptive field surrounds (Ito and Gilbert, 1999; Kapadia et al., 
1999; Sceniak et al., 1999; Pack et al., 2005; Huang et al., 2007; Roberts et al., 2007; see also Tadin et al., 2003). 


\subsection{Summary of the topics of this thesis}

Several physiological experiments have established that the effects of attention are well described by multiplicative modulation of single neuron responses, so that sensitivity but not selectivity is changed on the single neuron level. On the other hand, according to the biased competition model, attention biases the neuronal response in favor of an attended stimulus and reduces the influence of an unattended stimulus, possibly by contracting receptive fields around the attended stimulus. Such a change in receptive field size would correspond to a sharpening of the spatial tuning curve of a neuron, and would be a non-multiplicative change. In the first part of this thesis, changes of receptive field sizes and profiles in area MT were measured while attention was switched between two stimuli inside the receptive field.

Receptive field profiles consist not only of an excitatory center but often also include an inhibitory surround which plays an important role in image segmentation. The strength of surround inhibition has recently been shown to be highly variable with stimulus properties, perceptual context and also attention. In the second part of the thesis, we tested if attention modulates not only the overall inhibition strength but also the profile of the inhibitory part of the receptive field.

Non-multiplicative changes of receptive field profiles could be explained by a multiplicative modulation of inputs from a lower level that feed into the receptive field. The third part of the thesis provides a quantitative test of such a model in a theoretical study.

On the behavioral level, there has been controversy if attention improves performance by a reduction of noise or by an enhancement of the attended stimulus. Recently, several studies have shown that attention can change the perceptual quality of the attended stimulus. If the positions of receptive fields are used by the visual system to construct a representation of the spatial layout of the visual world, changes of receptive field profiles as observed in the physiological part of this thesis should lead to a change in the perception of spatial relations, 
specifically stimulus size. In the fourth part of the thesis, we tested the hypothesis that attention modulates perceived size. 


\section{Original articles and manuscripts}

This chapter contains the following research articles and manuscripts:

- Dynamic shifts of visual receptive fields in cortical area MT by spatial attention. Womelsdorf T, Anton-Erxleben K, Pieper F, Treue S (2006) Nature Neuroscience 9(9): 1156-1160.

- Attention modulates center-surround structure in area MT. Anton-Erxleben K, Stephan VM, Treue S, prepared for submission.

- Receptive field shift and shrinkage in macaque area MT through attentional gain modulation. Womelsdorf T, Tzvetanov T, Anton-Erxleben K, Treue S (in revision) Journal of Neuroscience.

- Attention changes perceived size of moving visual patterns. AntonErxleben K, Henrich C, Treue S (2007) Journal of Vision 7(11): 1-9. 



\subsection{Dynamic shifts of visual receptive fields in cortical area MT by spatial attention}

This study shows that attention shifts receptive field profiles in macaque area MT towards an attended stimulus. This shift is accompanied by a shrinkage of receptive fields, which is however small in magnitude. Shift and shrinkage both provide an exception to the general principle of multiplicative scaling because they correspond to changes in spatial selectivity on the single cell level. They might be a result of multiplicative scaling of lower-level inputs, though. 



\title{
Dynamic shifts of visual receptive fields in cortical area MT by spatial attention
}

\author{
Thilo Womelsdorf ${ }^{1,2}$, Katharina Anton-Erxleben ${ }^{2}$, Florian Pieper $^{2} \&$ Stefan Treue ${ }^{2}$
}

\begin{abstract}
Voluntary attention is the top-down selection process that focuses cortical processing resources on the most relevant sensory information. Spatial attention-that is, selection based on stimulus position-alters neuronal responsiveness throughout primate visual cortex. It has been hypothesized that it also changes receptive field profiles by shifting their centers toward attended locations and by shrinking them around attended stimuli. Here we examined, at high resolution, receptive fields in cortical area MT of rhesus macaque monkeys when their attention was directed to different locations within and outside these receptive fields. We found a shift of receptive fields, even far from the current location of attention, accompanied by a small amount of shrinkage. Thus, already in early extrastriate cortex, receptive fields are not static entities but are highly modifiable, enabling the dynamic allocation of processing resources to attended locations and supporting enhanced perception within the focus of attention by effectively increasing the local cortical magnification.
\end{abstract}

Vision at an attended location is faster, more accurate, and of higher spatial resolution and enhanced sensitivity for fine changes ${ }^{1-4}$. Stimuli outside this 'spotlight of attention' appear to have lower contrast or might not be perceived at all ${ }^{5,6}$. Physiologically, one well-investigated effect of attention in visual cortex is a multiplicative modulation of neuronal responses ${ }^{7-9}$. But not all observed effects of attentional modulation are clearly multiplicative ${ }^{10-11}$. Most prominently, this is the case for the effect observed when one of two stimuli inside the receptive field is attended to: attending to the stimulus that elicits the stronger sensory response when presented alone typically enhances responses, whereas attending to the less optimal stimulus reduces responses $^{12-14}$. It has been suggested ${ }^{12}$ that the neural basis of this differential, push-pull modulation of the respective effectiveness of each stimulus is a shrinkage of receptive fields around the attended stimuli. This would attenuate the influence of unattended stimuli at nearby locations. This influential hypothesis has never been empirically validated. Such changes in the profiles of receptive fields would have far-reaching consequences in successive areas of the cortical processing hierarchy ${ }^{15-18}$. In particular, it would provide higherorder areas with an almost exclusive representation of stimuli at the attended spatial location ${ }^{19}$.

\section{RESULTS}

Neuronal shifts with attention inside the receptive field

To investigate the influence of attention on receptive fields, we recorded from 78 neurons in cortical area MT of two macaque monkeys. Area MT is an early processing stage in the dorsal pathway and is central for the processing of visual motion information. Recordings were made while the monkeys' attention was directed to one of two stimuli (S1, S2) moving in the antipreferred direction inside the receptive field, or to a third stimulus (S3) positioned outside the receptive field (Fig. 1). We acquired high-resolution maps of a neuron's receptive field by presenting a succession of brief probe stimuli at up to 52 positions covering the receptive field (sparing the locations of S1 and S2) while the monkey's attention was allocated to one of the three stimuli. Probe stimuli were of the same size as $\mathrm{S} 1, \mathrm{~S} 2$ and $\mathrm{S} 3$ but of higher contrast, and they moved in the preferred direction of the neuron. We conjectured that the hypothesized distortion of the receptive field would result in a push-pull effect, enhancing probe responses around the attended location and reducing responses to the probe farther from this focus of attention.

The results for an example neuron (Fig. 2; see also Supplementary Fig. 1 online) illustrate that the most responsive part of the receptive field was shifted substantially toward the attended position inside the receptive field (that is, when attention was directed toward S1 and S2, which were located inside the receptive field). To quantify this effect across our sample of $78 \mathrm{MT}$ neurons, we determined the 'neuronal shift': the amount by which the center of mass of each receptive field shifted between the conditions when attention was directed to $\mathrm{S} 1$ versus S2 along the axis of the 'attentional shift' (that is, the connection between the locations of S1 and S2). Positive values indicated shifts in the same direction as the attentional shift (that is, toward the focus of attention; Fig. 3a). Across our cells, we found a highly significant neuronal shift that averaged $30.3 \%$ of the attentional shift (Fig. $3 \mathbf{b}$ horizontal axis, $P<0.001, t=14.0$, one-sample $t$-test). For the sample of receptive field sizes and stimulus locations in our study, this corresponded to an average shift of $3.0^{\circ}$ of visual angle or $22 \%$ of the receptive field diameter. Additionally, we determined the shift for the orthogonal direction (with positive values indicating shifts toward the

\footnotetext{
${ }^{1}$ F.C. Donders Centre for Cognitive Neuroimaging, Radboud University, Kapittelweg 29, 6525 EN Nijmegen, The Netherlands. ${ }^{2}$ Cognitive Neuroscience Laboratory, German
} Primate Center, Kellnerweg 4, 37077 Göttingen, Germany. Correspondence should be addressed to T.W. (t.womelsdorf@fcdonders.ru.nl).

Received 7 June; accepted 20 July; published online 13 August 2006; doi:10.1038/nn1748 
ARTICLES

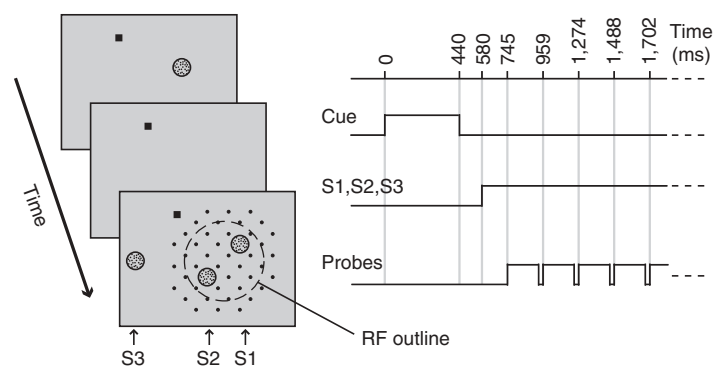

Figure 1 Experimental protocol. Time course of events and example of the placement of cue, stimuli and probes in an experimental trial (details in Methods). Black square, fixation point.

fovea) as a measure of the variability of the data. This distribution showed no significant bias (Fig. $3 \mathbf{b}$ vertical axis, $P=0.48$ ).

To determine how many of the individual cells showed a significant shift, we performed a bootstrap analysis (Supplementary Note online) Of our 78 cells, $49(63 \%)$ showed a significant $(P<0.05)$ receptive field shift in the direction of the attentional shift, and none shifted significantly in the opposite direction. In contrast, the orthogonal shift was significant $(P<0.05)$ in only 3 cells $(3.8 \%)$, indicating that the receptive fields displacement fell along the axis of the attentional shift.

We can rule out the possibility that the neuronal shift was due to small differences in eye position across conditions, because we calculated the deviation in eye position along the axis of the attentional shift and found an average displacement of only $0.0182^{\circ}\left( \pm 0.0065^{\circ}\right.$, s.e.m.; details in Supplementary Note and Supplementary Fig. 2 online).

\section{Receptive field size changes with attention}

To determine if the neuronal shift was accompanied by the hypothesized shrinkage of the receptive field around the attended stimulus, we compared the size of receptive fields when attention was directed inside versus outside the receptive field. Whereas receptive fields were, on average, $4.3 \%$ smaller with attention inside the receptive field (Fig. 3c), this effect was only marginally significant $( \pm 3.4 \%, 95 \%$ confidence interval, $P<0.05, t=-2.56$, paired $t$-test). Receptive field shrinkage was isotropic - that is, similar in magnitude parallel and orthogonal to the direction of the attention shift (Supplementary Note and
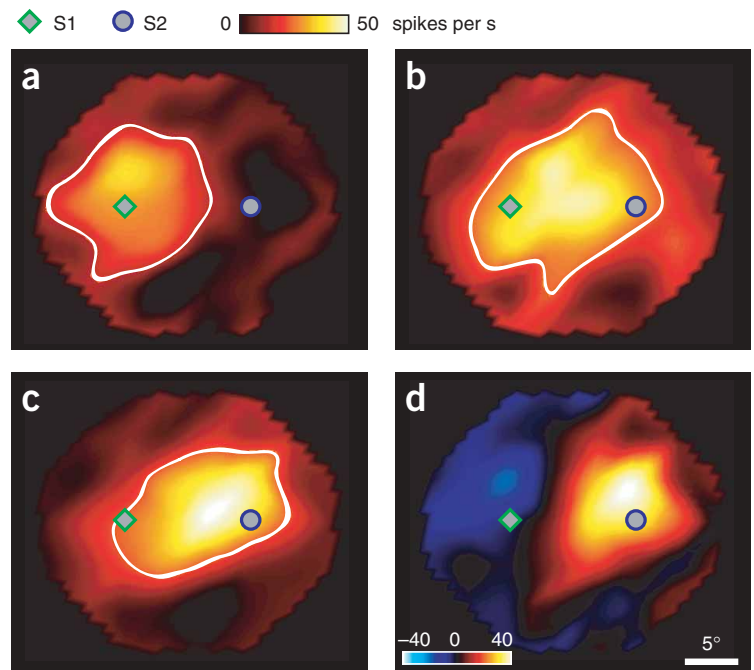

Supplementary Fig. 3 online). Thus, the influence of spatial attention was dominated by a shift, rather than a shrinkage, of receptive fields, and our observation of only a small amount of shrinkage resonates with the psychophysical observation of a coarse spatial resolution of visual attention ${ }^{20}$.

Our findings demonstrate that the enhanced/reduced response of MT neurons when spatial attention is directed to the preferred/ antipreferred of two stimuli inside their receptive field can be accounted for by a systematic and large change in the receptive field profile. In effect, attention changes the spatial filtering characteristics of those MT neurons whose receptive fields overlap with the currently attended location.

\section{Spatial extent of receptive field shifts with attention}

For an attentional location just outside the receptive field, a previous study in area V4 (ref. 21) has demonstrated that receptive fields are distorted toward the location of attention. We were wondering if such an effect is also present in area MT and if it extends beyond the immediate surround of the receptive field. To investigate this issue, we compared the receptive field profile when attention was directed to either S1 or S2, located inside the receptive field (the 'in' condition), to the profile when attention was directed to S3, located far outside the receptive field in the opposite hemifield (the 'out' condition; Fig. 4a). If the receptive field in the out condition was unaffected by the location of the attentional focus relative to the receptive field, the in condition should shift the receptive field center along a vector pointing directly at the attended stimulus' location inside the receptive field. If, on the other hand, the receptive field center in the out condition was already attracted toward the attended stimulus' location outside the receptive field, then switching to the in condition should not only shift the center to the new location of attention, but should also release it from the attraction toward the S3 location. In this case, the resulting shift vector should not point directly at the attended stimulus' location inside the receptive field, but should be deviated somewhat by a vector component pointing away from the direction of $\mathrm{S} 3$. We found just that for a significant majority of the vectors $(62.5 \%, P<0.05$, Wilcoxon signed rank test; Fig. 4b). Further support for a shift of the receptive field center toward the location of attention in the out condition came from the finding that the eccentricities of the receptive fields in trials with attention outside the receptive field were smaller than their eccentricities in trials with attention inside the receptive field $(7.9 \%, P=0.019$, paired $t$-test). These findings are not only in agreement with the findings from V4, but go well beyond them in demonstrating a far-reaching effect of spatial attention that even affects neurons with receptive fields in the opposite hemifield.

\section{DISCUSSION}

One effect of spatial attention that has been reported frequently is a multiplicative modulation of tuning curves ${ }^{7-9}$. The push-pull modulation of MT receptive fields with shifts of spatial attention within the

Figure 2 Receptive field profiles of an example cell, as 2D surface plots. (a-c) Receptive field (RF) profiles when attention was directed inside the RF, to stimulus S1 (a) or S2 (c), or when attention was directed outside the RF, to S3 (b). The surface color at each point in the plots indicates the increase in the neuron's response elicited by the presentation of a probe stimulus at that position, over the response observed in the absence of a probe (that is, when only S1 and S2 were present). Supplementary Fig. 1 online shows the same data as absolute firing rates. (d) Difference map, computed by subtracting the RF when attention was on S1 from the RF when attention was on S2. The map illustrates that shifting attention from S1 to S2 enhances responsiveness around S2 and reduces it near S1. 


\section{ARTICLES}

Figure 3 Quantification of RF shift and shrinkage. (a) Convention used to quantify the shift of RF centers. (b) Magnitudes of the neural shifts, along the axis of the attentional shift ( $x$-axis and top histogram) or orthogonal to it ( $y$-axis and right histogram; positive values indicate shifts toward the fovea). Light gray, monkey D; dark gray, monkey R. Circle, example cell illustrated in Figure 2 (attentional shift: $+53.6 \%$.). Filled and unfilled histogram bars and symbols indicate significant $(P<0.05)$ and nonsignificant shifts, respectively. The top histogram shows a highly significant mean neuronal shift toward the attended stimulus $(30.3 \% \pm 4.3 \%(95 \% \mathrm{Cl})$, $P<0.001)$. This mean shift was larger in monkey $\mathrm{R}(35 \% \pm 4.8 \%)$ than in monkey $\mathrm{D}(18 \% \pm 6.2 \%)$. There was no significant bias in the direction orthogonal to the direction of the attentional shift (mean: $1.3 \% \pm 3.5 \%$ ). (c) Histogram of RF size changes when attention was directed outside versus inside the RF. Stars, size change of the example cell from Figure 2; the RF shrank when attention was directed toward S1 or S2 (-9.5\% and $-4.4 \%$, respectively). The histogram is shifted slightly to the left, indicating a small but significant mean reduction of RF size $(4.3 \pm 3.4 \%, 95 \% \mathrm{Cl}, P<0.05$, paired $t$-test) when attention was directed into the RF. The size reduction did not differ significantly between the two monkeys and was around the 0.05 significance level when the two data sets were analyzed separately (monkey R: $-3.7 \% \pm 4.3 \%, P=0.077$; monkey $\mathrm{D}:-5.7 \% \pm 5.6 \%, P=0.039$ ).

receptive field is not a multiplicative change of a neuron's spatial tuning curve; nevertheless, it is important to point out that the underlying attentional modulation might still be multiplicative. If attention differentially acts on the neurons with smaller receptive fields that provide the input to MT, the observed modulation in receptive field profiles could be achieved with multiplicative effects: for instance, by increasing the response gain of input neurons representing the attended location and decreasing the response gain of the neurons representing unattended regions within the MT receptive field ${ }^{15}$.

Our findings show that spatial attention shifts the receptive fields of MT neurons toward the attentional focus. Such a dynamic routing provides a powerful mechanism to increase selectivity of visual representations within and across functionally specialized visual areas, and serves to constrain models of the perceptual organization of selective visual processing. For the neural population as a whole, the spatial shift reflects the additional recruitment of processing resources at the focus of attention. Paralleling the increase in the observed magnitude of attentional modulation, the shifts of receptive fields probably increase with increasing receptive field size in successive areas of the visual hierarchy. It could also be the neural correlate of various perceptual effects that are centered on the focus of attention, including enhanced processing accuracy and spatial resolution close to the attentional focus, suppression in its surround and distortions in spatial judgments ${ }^{22-25}$. a

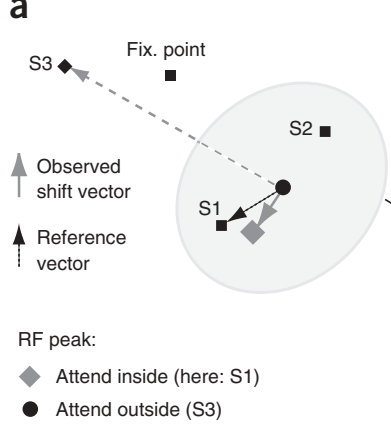

b Attended stim.

inside RF (S1 / S2)

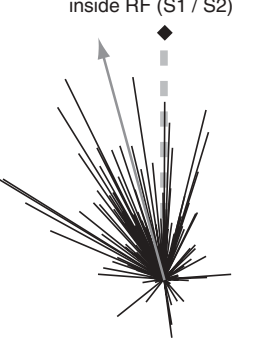

In summary, our finding that receptive fields are highly malleable by the attentional state demonstrates a dynamic spatial filtering system that could provide the neuronal correlate of the central purpose of attentional modulation: namely, the allocation of processing resources to the attended stimuli at the expense of the unattended ones. This dynamic modification by spatial attention seems to affect the receptive field mosaic across the whole visual field and is likely to be part of a mechanism active during the planning or execution of eye movements ${ }^{26-29}$. Furthermore, the similarity between the far-reaching influence of spatial attention across the visual field and the distribution of feature-based attention ${ }^{7}$ supports the hypothesis that both rely on a common underlying mechanism.

\section{METHODS}

Electrophysiological recording. All procedures reported in this study were approved by the district government of Braunschweig, Lower Saxony, Germany. Neuronal activity was recorded from 57 and 21 single isolated cells from

Figure 4 Receptive field shift when attention is directed inside versus outside the receptive field. (a) Typical stimulus arrangement. Black circle and gray diamond, RF center positions. Vectors indicate the direction of a direct shift of the RF center toward the attended stimulus inside the RF (the 'reference vector') and the direction of the observed shift. Note that the eccentricity of the RF when attention was directed inside the RF (distance from fixation point to gray square) was, on average, $7.9 \%$ larger than the RF eccentricity when attention was directed to S3 (distance from fixation point to black circle). We analyzed the data from the two monkeys separately and found that this effect was significant only in monkey $\mathrm{R}(9.9 \%, P=0.02)$. (b) Distribution of observed shift vectors. These data are based on the 64 cells for which sufficient data were available for both the attend-outside (to stimulus S3) and each of the attend-inside (to stimulus S1 or S2) conditions. All vectors were rotated, such that the reference vector would point straight up, and flipped horizontally (if necessary), such that the S3 stimulus position would be on the right. The gray arrow pointing in the direction opposite to the location of S3 indicates a systematic and significant deviation of the vectors (average deviation of $15.2^{\circ}, 95 \%$ angular confidence: $\left.\pm 9.8^{\circ}, P<0.05\right)$, and implies a far-reaching modulation of receptive fields by attention. We analyzed the data from the two monkeys separately and found that this effect was significant only in monkey $\mathrm{R}\left(19.2 \pm 10.5^{\circ}, P<0.05\right)$. 
ARTICLES

monkeys $\mathrm{R}$ and $\mathrm{D}$, respectively, with tungsten electrodes (impedance 1.0-4.0 M $\Omega$, Frederick Haer). Cell isolation was based on window discrimination (BAK Electronics or Plexon). Cells were localized in area MT by their physiological characteristics and the histological reconstruction of recording sites in monkey R. Access to MT was provided by a craniotomy and a recording chamber surgically implanted above the superior temporal sulcus of the left hemisphere. During the experiment, a custom computer program running on an Apple Macintosh PowerPC controlled stimulus presentation, and monitored and recorded eye positions and neuronal and behavioral responses. Eye positions were determined using a high-resolution, video-based eye tracking system (ET49, Thomas Recording $\mathrm{GmbH}$ ) with a sampling frequency of 230 $\mathrm{Hz}$, and were digitized and stored at $200 \mathrm{~Hz}$.

Visual stimuli. Stimuli were moving random dot patterns (RDPs) of smal bright dots (density: 10 dots per $\mathrm{deg}^{2}$ ) plotted within a stationary circular aperture on a dark $(0.7 \mathrm{~cd})$ computer monitor. For each receptive field, stimul S1 and S2 were placed at similarly responsive positions in the receptive field at equal eccentricity and equidistant from the center of the receptive field when the monkey was directing its attention to the fixation point. S3 was placed in the opposite hemifield. Initial estimation of this 'sensory' receptive field center was based on a manual mapping with a mouse-controlled RDP and with quantitative mapping during the main experiment. Note that the shift and shrinkage of the receptive field when attention was directed to S1 or S2 could cause the other stimulus to fall outside the receptive field on those trials (see Fig. 2a-c for an example). Note also that the initial manual mapping was used only for the placement of the stimuli. All receptive fields profiles used in the analysis were mapped quantitatively during temporally interleaved trials in which attention was directed to S1, S2, S3 or the fixation point. Therefore al receptive field profiles used in the analysis were determined with temporally interleaved mapping. This ensured that the results were not contaminated by potential changes in a neuron's isolation or responsiveness over time.

Stimuli S1, S2 and S3 moved in the cell's antipreferred direction and with a reduced luminance $(19 \mathrm{~cd})$ in order to prevent a saturation of the cell's response due to the presence of S1 and S2 alone. A fourth RDP (the 'probe', $47 \mathrm{~cd}$ ) moving in the preferred direction of the neuron was used to probe the spatial sensitivity. This probe stimulus was of the same size as S1, S2, and S3, and was presented at the intersections of a dense grid (between 42 and 52 positions) spanning the classical receptive field and its immediate surround, but sparing the S1 and S2 location (in order to avoid potential nonlinear interactions and changes in the sensory quality of S1 and S2 that could affect the attentional task performed on these stimuli). The longer axis of the elliptical probe grid was always along the S1-S2 axis.

Experimental procedure. First we isolated a single cell and determined it preferred direction. Then we centered the virtual grid of the array of probe positions at the estimated center of the receptive field (Fig. 1), in an approach similar to that used in a previous study ${ }^{30}$. A trial started once the monkey's gaze was directed within $0.75^{\circ}$ of the fixation point. After the monkey touched a lever, the cue (a stationary RDP) appeared for $445 \mathrm{~ms}$ at the upcoming position of either S1, S2 or S3, indicating the 'target' location for the trial. After a 145-m interstimulus interval, S1, S2 and S3 were presented. The task was to detect a brief $(80 \mathrm{~ms}$ ) phase during which the target (the stimulus at the previously cued location) moved in a different direction, while ignoring equivalent phases in the two other RDPs (the 'distracters'). Successive presentation of the receptive field probe stimulus began $160 \mathrm{~ms}$ after the onset of S1, S2 and S3. Probe duration was $187 \mathrm{~ms}$ with an interprobe blank period of $27 \mathrm{~ms}$. The direction of the target and the distracter stimuli changed 670-4,670 ms after the trial began; the times of these changes were randomly picked from a uniform distribution. In control trials, the monkey had to detect a change in the color of the fixation square. Trials were aborted if the monkey's gaze left the fixation window or if the monkey released the lever outside a 150 - to 750 -ms time window after the change of the target stimulus (for example, because the monkey released the lever in response to a distracter change, or if it failed to detect the target change).

Data analysis. To analyze the data, we used the mean neuronal responses to probe presentations from only the correctly completed trials in the three experimental conditions (that is, when attention was directed to S1 ('attend- inside' receptive field), to S2 ('attend-inside' receptive field) or to S3 ('attendoutside' receptive field)). The mean firing rate was computed for a 60 - to 200-ms interval after the onset of the probe stimulus. For each condition, some of the probe presentations were skipped (that is, no probe was shown) in order to determine the cell's response to S1 and S2 alone. This baseline was subtracted from all probe responses before the interpolation of the receptive field profile by cubic spline interpolation. These two-dimensional (2D) profiles were used to determine receptive field centers and sizes.

We calculated the shift of the receptive field (Fig. 3b) between attentional conditions by using the center of mass of one-dimensional projections of the receptive field surface. For this analysis, we averaged the activity of the receptive field profile orthogonal to the axis connecting the two stimuli within the receptive field (Fig. 3b, horizontal axis) or to an orthogonal axis (Fig. $\mathbf{3 b}$, vertical axis). Averaging was limited to regions of the receptive field surface that exceeded two s.d. of the baseline response in any of the attentional conditions, in order to exclude visual field regions that did not contribute to the receptive field profile. For each $2 \mathrm{D}$ projected receptive field slice, we determined the center of mass and the peak positions (data not shown but qualitatively identical to results with the center of mass). Receptive field shifts between the two attend-inside conditions are expressed as the proportional distance of the center of mass relative to the reference distance between the stimuli S1 and S2 (Fig. 3a). Shift values were positive when the receptive field center lay closer to the attended stimulus in the respective attentional conditions.

To quantify the statistical significance of the shift of the neuronal receptive fields for individual cells, we applied a bootstrap method (details in Supplementary Note)

The receptive field size was calculated as the square root of the area in which the $2 \mathrm{D}$ receptive field surface exceeded the half-maximal response (after subtracting the baseline responses- that is, responses in the same attentional condition when S1 and S2 were present but no probe stimulus was shown). We compared receptive field sizes when attention was directed to the stimulus outside the receptive field versus when attention was directed to either of the stimuli inside the receptive field, by using an attentional modulation index ${ }^{13}$. $\left(S_{\text {in }}-S_{\text {out }}\right) /\left(S_{\text {in }}+S_{\text {out }}\right)$, where $S_{\text {in }}$ and $S_{\text {out }}$ are the size of the receptive field when attention was directed inside and outside, respectively. The size index ranges between -1 and 1; negative values reflect a smaller receptive field size when attention was directed inside the receptive field compared to outside the receptive field (Fig. 3c). The average index value is a conservative estimate as it corresponds to a geometric mean (that is, the mean is less influenced by large values than a regular mean would be).

Note: Supplementary information is available on the Nature Neuroscience website.

\section{ACKNOWLEDGMENTS}

We thank T. Tzvetanov and M. Husain for discussions in the course of this study; U. Grosshennig, D. Prüsse, K. Fahrner and R. Rode-Brockhausen for technical assistance; and D. Heller-Schmerold and S. Stuber for administrative assistance. This work was supported by the German Research Foundation (SFB 550 \& GRK 289) and the Bernstein Center for Computational Neuroscience, Göttingen (Federal Ministry of Education and Research grant 01GQ0433).

\section{AUTHOR CONTRIBUTIONS}

T.W. and S.T. conceived the experiment and performed the data analysis. T.W. and K.A.-E. performed the experiments. F.P. provided technical assistance and helped during the experiment. T.W. and S.T. wrote the paper.

\section{COMPETING INTERESTS STATEMENT}

The authors declare that they have no competing financial interests.

Published online at http://www.nature.com/natureneuroscience

Reprints and permissions information is available online at http://npg.nature.com/ reprintsandpermissions/

1. Yeshurun, Y. \& Carrasco, M. Attention improves or impairs visual performance by enhancing spatial resolution. Nature 396, 72-75 (1998).

2. He, S., Cavanagh, P. \& Intriligator, J. Attentional resolution and the locus of visual awareness. Nature 383, 334-337 (1996).

3. Hawkins, H.L. et al. Visual attention modulates signal detectability. J. Exp. Psychol. Hum. Percept. Perform. 16, 802-811 (1990). 


\section{ARTICLES}

4. Carrasco, M., Williams, P.E. \& Yeshurun, Y. Covert attention increases spatial resolution with or without masks: support for signal enhancement. J. Vis. 2, 467-479 (2002).

5. Carrasco, M., Ling, S. \& Read, S. Attention alters appearance. Nat. Neurosci. 7 , 308-313 (2004).

6. Simons, D.J. \& Rensink, R.A. Change blindness: past, present, and future. Trends Cogn. Sci. 9, 16-20 (2005).

7. Treue, S. \& Martinez Trujillo, J.C. Feature-based attention influences motion processing gain in macaque visual cortex. Nature 399, 575-579 (1999).

8. McAdams, C.J. \& Maunsell, J.H. Effects of attention on orientation-tuning functions of single neurons in macaque cortical area V4. J. Neurosci. 19, 431-441 (1999).

9. Martinez-Trujillo, J.C. \& Treue, S. Feature-based attention increases the selectivity of population responses in primate visual cortex. Curr. Biol. 14, 744-751 (2004).

10. Reynolds, J.H. \& Chelazzi, L. Attentional modulation of visual processing. Annu. Rev. Neurosci. 27, 611-647 (2004)

11. Treue, S. Neural correlates of attention in primate visual cortex. Trends Neurosci. 24, 295-300 (2001).

12. Moran, J. \& Desimone, R. Selective attention gates visual processing in the extrastriate cortex. Science 229, 782-784 (1985)

13. Treue, S. \& Maunsell, J.H. Effects of attention on the processing of motion in macaque middle temporal and medial superior temporal visual cortical areas. J. Neurosci. 19, 7591-7602 (1999).

14. Reynolds, J.H., Chelazzi, L. \& Desimone, R. Competitive mechanisms subserve attention in macaque areas V2 and V4. J. Neurosci. 19, 1736-1753 (1999).

15. Maunsell, J.H.R. \& McAdams, C.J. Effects of attention on the responsiveness and selectivity of individual neurons in visual cerebral cortex. in Visual Attention and Cortical Circuits (eds. Braun, J., Koch, C. \& Davis, J.L.) Ch. 6 103-120 (MIT Press, Cambridge, Massachusetts, 2001).

16. Reynolds, J.H. \& Desimone, R. The role of neural mechanisms of attention in solving the binding problem. Neuron 24, 19-29 (1999).

17. Salinas, E. \& Abbott, L.F. A model of multiplicative neural responses in parietal cortex Proc. Natl. Acad. Sci. USA 93, 11956-11961 (1996).
18. Itti, L. \& Koch, C. Computational modelling of visual attention. Nat. Rev. Neurosci. 2, 194-203 (2001).

19. Everling, S., Tinsley, C., Gaffan, D. \& Duncan, J. Filtering of neural signals by focused attention in the monkey prefrontal cortex. Nat. Neurosci. 5, 671-676 (2002).

20. Intriligator, J. \& Cavanagh, P. The spatial resolution of visual attention. Cognit. Psychol. 43, 171-216 (2001).

21. Connor, C.E., Preddie, D.C., Gallant, J.L. \& Van Essen, D.C. Spatial attention effects in macaque area V4. J. Neurosci. 17, 3201-3214 (1997).

22. Suzuki, S. \& Cavanagh, P. Focussed attention distorts visual space: an attentional repulsion effect. J. Exp. Psychol. Hum. Percept. Perform. 23, 443-463 (1997)

23. LaBerge, D., Carlson, R.L., Williams, J.K. \& Bunney, B.G. Shifting attention in visual space: tests of moving-spotlight models versus an activity-distribution model. J. Exp. Psychol. Hum. Percept. Perform. 23, 1380-1392 (1997).

24. Müsseler, J., Stork, S. \& Kerzel, D. Comparing mislocalizations with moving stimuli. The Fröhlich effect, the flash-lag effect and representational momentum. Vis. cogn. $\mathbf{9}$ 120-138 (2002).

25. Tsal, Y. \& Bareket, T. Effects of attention on localization of stimuli in the visual field. Psychon. Bull. Rev. 6, 292-296 (1999).

26. Ben Hamed, S., Duhamel, J R., Bremmer, F. \& Graf, W. Visual receptive field modulation in the lateral intraprietal area during attentive fixation and free gaze. Cereb. Cortex 12, 234-245 (2002)

27. Duhamel, J.R., Colby, C.L. \& Goldberg, M.E. The updating of the representation of visual space in parietal cortex by intended eye movements. Science 255, 90-92 (1992).

28. Tolias, A.S. et al. Eye movements modulate visual receptive fields of V4 neurons. Neuron 29, 757-767 (2001).

29. Krekelberg B. Kubischik, M., Hoffmann, K.P. \& Bremmer, F. Neural correlates of visual localisation and perisaccadic mislocalisation. Neuron 37, 537-545 (2003).

30. Britten, K.H. \& Heuer, H.W. Spatial summation in the receptive fields of MT neurons. J. Neurosci. 19, 5074-5084 (1999). 


\title{
Supplementary Information for
}

\author{
Dynamic shifts of visual receptive fields \\ in MT by spatial attention
}

\author{
Thilo Womelsdorf ${ }^{1,2}$, Katharina Anton-Erxleben ${ }^{2}$, Florian Pieper $^{2}$, Stefan Treue ${ }^{2}$ \\ ${ }^{1}$ F.C. Donders Centre for Cognitive Neuroimaging, Radboud University, Kapittelweg 29, 6525 EN Nijmegen, \\ The Netherlands \\ ${ }^{2}$ Cognitive Neuroscience Laboratory, German Primate Center, Kellnerweg 4, 37077 Göttingen, Germany
}

The supplementary material provides additional information about three results:

- We address the possibility that the attentional shift effects observed were caused by changes in eye position.

- We provide information about the bootstrap method used to estimate the statistical reliability of the receptive field centre shift in individual neurons.

- We illustrate that the moderate shrinkage of receptive fields in conditions with attention inside versus outside the receptive field is isotropic, i.e. it is not biased with regard to the direction of attention.

The receptive field shift cannot be explained by changes of the eye position within the fixation window

Receptive fields in area MT are retinotopic, i.e. they move across the visual field with changes in eye position. It is therefore conceivable that the shifts in receptive field centres we have observed are due to systematic differences in eye positions between the attentional conditions. Such a shift would only be able to provide a small contribution to the changes we have observed since the average shift of receptive field centres was $3.0^{\circ}$ (see main text, Fig. 3), much larger than the diameter of the fixation window $\left(1.5^{\circ}\right)$ that the monkey had to stay within throughout all trials. Nevertheless, we computed the difference of the average eye position in conditions with attention to either stimulus $\mathrm{S} 1$ or stimulus $\mathrm{S} 2$ in the direction from $\mathrm{S} 1$ to $\mathrm{S} 2$, i.e. the axis along which attention was shifted between conditions. This analysis revealed a very small difference in average eye position of only $0.018^{\circ}\left( \pm 0.0065^{\circ} \mathrm{SE}\right)$ between the conditions. We also calculated the average eye position difference between the 
attend-inside conditions and the attend-outside condition along the axis of the respective stimuli (i.e. along the axis from S1-to-S3 or from S2-to-S3). Similar to the previous analysis we found a very small difference of eye positions of only $0.009^{\circ}\left( \pm 0.004^{\circ} \mathrm{SE}\right)$ in the direction of the attended stimulus. Supplementary Figure 2 illustrates the distribution of gaze positions for three representative example cells in conditions with attention to either stimulus S1 (upper panels), or to S2 (lower panels) inside the receptive field. The centre of the coordinate system is the fixation point and the relative direction of the attended (nonattended) stimulus is indicated by the square (circle) plotted on the axes border. For these examples, the average difference of gaze positions along the S1-to-S2 axis ranged from $-0.036^{\circ}$ to $0.054^{\circ}$. The receptive field shifts of these conditions were $1.8^{\circ}$ to $4.2^{\circ}$ in the direction of the attended stimulus, many times larger than the differences in average gaze position. These results demonstrate that the contribution of eye position artefacts to the estimated receptive field shifts was marginal at best.

\section{Calculation of the statistical significance of the receptive field shift in individual cells}

To quantify the statistical significance of the shift of the neuronal receptive fields for individual cells we applied a bootstrap method. To this end we computed the variability of the receptive field profile by simulating our experiment 1000 times. On each repetition we simulated the response for each probe position by drawing (with replacement) and averaging across as many individual responses as were collected in the actual experiment from the original distribution of responses. To compare the receptive field centers for the two attention conditions (attend S1 versus attend S2) statistically we determined the $95 \%$ range of receptive field centers for each condition (by determining the $2.5 \%$ leftmost and $2.5 \%$ rightmost center position from the 1000 simulations). When the $95 \%$ distributions of both conditions did not overlap the difference in receptive field center positions were considered statistically significant. This procedure corresponds to a two-tailed randomization test with a significance level of $\mathrm{P}=0.05$.

\section{Spatial isotropy of changes in receptive field size with attention inside the receptive field}

We found only moderate receptive field shrinkage with attention to a stimulus inside compared to attention outside the receptive field (see Fig. 3c). On average receptive fields were $4.3 \%$ smaller when attention was directed inside the receptive field with a $95 \%$ confidence range of $\pm 3.4 \%$. To determine whether this change in receptive field size was 
different in the direction of the attention shift (see Fig. 3a and Fig. 4) we computed the maximum extent of the receptive field parallel and orthogonal to the axis of the attentional shift, i.e. the axis from stimulus S3 outside the receptive field (the attended stimulus in the outside condition) toward stimulus S1 / S2 in conditions with attention inside the receptive field. This analysis showed no spatial bias of receptive field size changes parallel and orthogonal to the direction of the attentional shift (paired t-test, $\mathrm{P}=0.444, \mathrm{t}=0.77$ ). The distribution of receptive field size changes parallel to the attentional shift (along the $\mathrm{x}$-axis) and orthogonal to the axis of the attentional shift (along the y-axis) are shown in Supplementary Figure 3. 


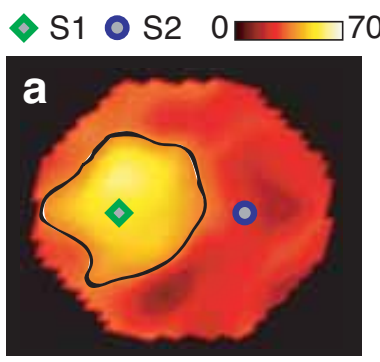

70 spikes per s
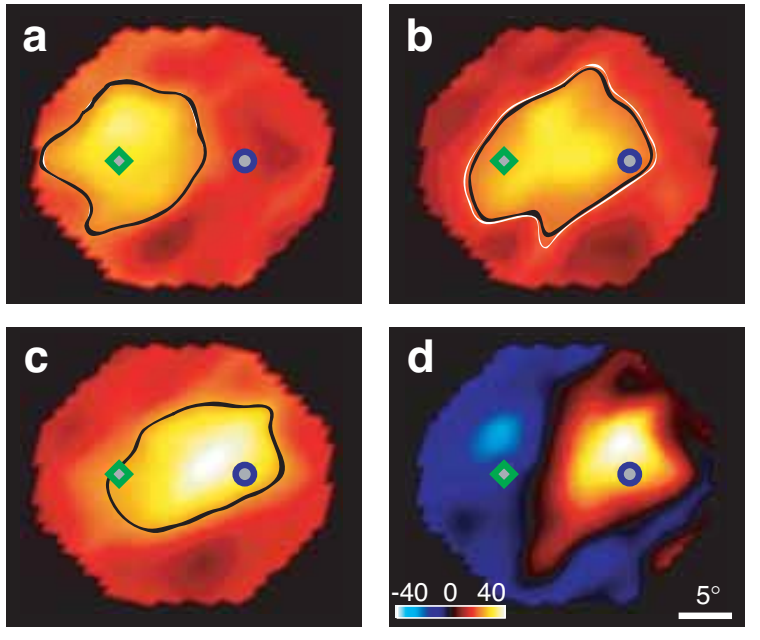

Supplementary Figure 1. Receptive field profiles of an example cell as 2dimensional surface plots (same format as in Fig. 2). The RF profiles are from the same example cell as shown in (Fig. 2). Here the plots are based on the raw response evoked during probe presentation, without subtracting the response evoked by S1 and S2 alone. The black outlines represent the area above half-maximum response and the white outline is based on the data in (Fig. 2).

Womelsdorf, Anton-Erxleben, Pieper \& Treue Supplementary Fig. 1 


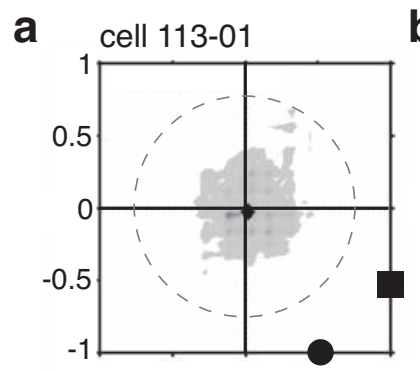

b cell 060-02
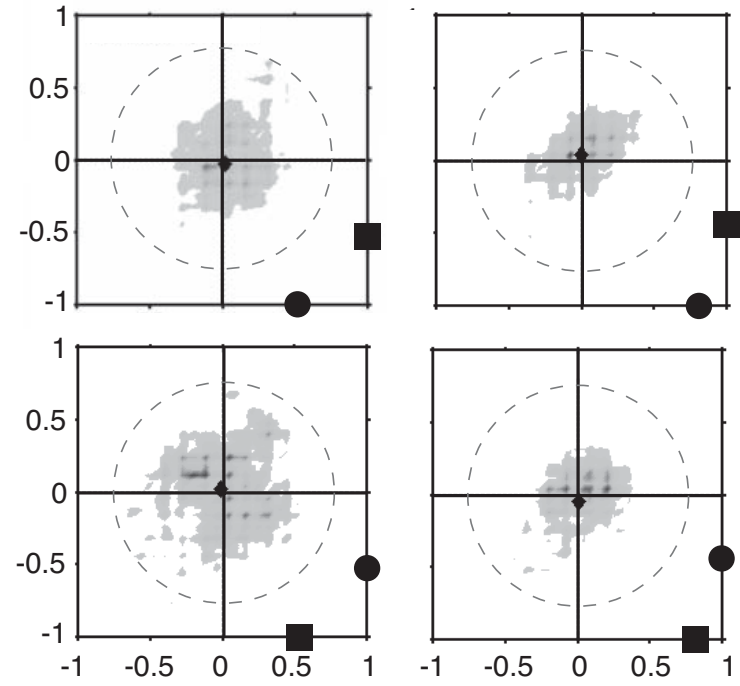

mean diff. on

S1-S2 axis :

RF shift (\%):

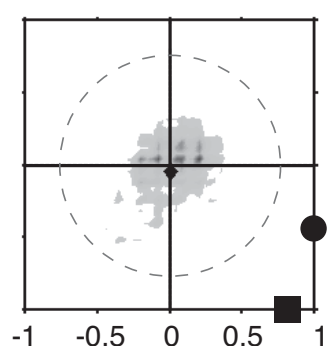

$0.054^{\circ}$

$1.8^{\circ}$

$(25.0 \%)$
C cell 114-01
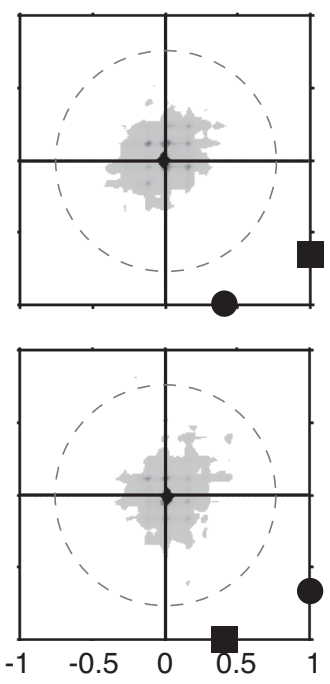

$-0.026^{\circ}$

$1.9^{\circ}$

(14.6\%)

Supplementary Figure 2. Distribution of eye positions for trials where attention was directed onto one of the stimuli inside the receptive field in three example cells (a-c) The grey values represent the frequency of gaze positions relative to the fixation square represented by the central intersection. The relative direction from the fixation point to the attended (non-attended) stimulus is shown as a square (circle) on the axis border (see text for details). Axes values represent the distance of eye position from the fixation point in degrees of visual angle. The circles around the plot centres represent the virtual fixation window within which the monkey's gaze had to stay throughout each trial.

\section{Womelsdorf, Anton-Erxleben, Pieper \& Treue Supplementary Fig. 2}




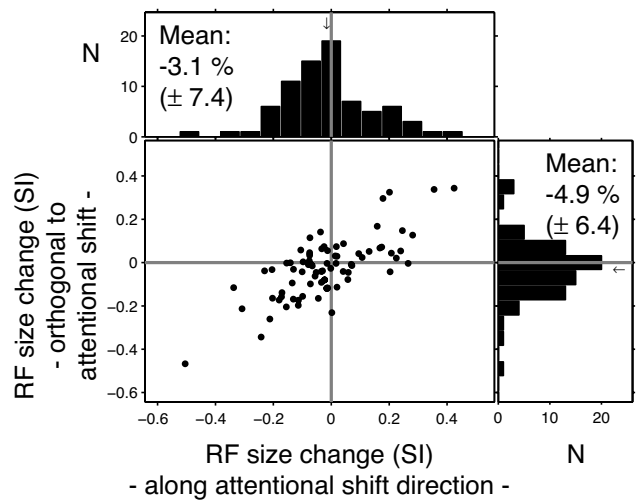

Supplementary Figure 3. Spatial isotropy of changes in receptive field size with attention inside (to stimulus $\mathrm{S} 1$ or S2) versus attention outside the receptive field (to stimulus S3). Shown is the distribution of receptive field size changes parallel (along the $\mathrm{x}$-axis) and orthogonal (along the $\mathrm{y}$-axis) to the axis from stimulus $\mathrm{S} 3$ to stimulus $\mathrm{S} 1 / \mathrm{S} 2$ for conditions with attention to $\mathrm{S} 3$ and attention to $\mathrm{S} 1 / \mathrm{S} 2$. The receptive field size change was not statistically different parallel versus orthogonal to the axis of the attention shift (3.1\% versus $4.9 \%$ respectively).

\section{Womelsdorf, Anton-Erxleben, Pieper \& Treue Supplementary Fig. 3}





\subsection{Attention modulates center-surround structure in area MT}

This study shows that not only the excitatory but also the inhibitory regions of receptive field profiles in area MT are shifted towards an attended stimulus. Such an enhanced inhibition near the attentional focus might contribute to the segregation of the attended stimulus from distractors and thus increase spatial resolution. In addition, we find evidence for a change from segregative to integrative receptive field properties depending on the distance between the receptive field center and the attentional focus. 



\title{
ATTENTION MODULATES CENTER-SURROUND STRUCTURE IN AREA MT
}

\author{
Katharina Anton-Erxleben, Valeska M. Stephan, Stefan Treue \\ Cognitive Neuroscience Laboratory, German Primate Center, Kellnerweg 4, \\ 37077 Göttingen, Germany
}

\begin{abstract}
Directing spatial attention to a location inside the classical receptive field (CRF) of a neuron in macaque medial temporal area (MT) has been shown to shift the center of the CRF towards the attended location. Here we investigate the influence of spatial attention on the profile of the inhibitory surround present in many MT neurons. Two monkeys were trained to attend to the fixation point or to one of two random dot patterns (RDPs) placed at equal eccentricity inside or besides the cRF, while a third RDP (the probe) was presented in quick succession at regularly spaced positions spanning the receptive field center and surround. Mean responses to probe presentation at the different positions were used to compute a map of the excitatory receptive field and its inhibitory surround. We find a significant shift of the center of the CRF and of the inhibitory surround profile towards the attended stimulus. The surround shift cannot be explained by the covering / uncovering of inhibitory regions due to the center shift; instead, attention modulates the surround itself. An enhanced inhibition near the attentional focus might contribute to the segregation of the attended stimulus from distractors and thus increase spatial resolution. In addition, attention inside the receptive field shrinks the cRF and tends to enhance surround suppression, while attention to a spot besides the receptive field expands the CRF diameter, reduces surround inhibition and increases CRF amplitude. This change in spatial summation might serve to strengthen the representation of the attended stimulus while reducing influences from distractors. Both shifts and summation changes represent a non-multiplicative modulation of the receptive field's center-surround structure by attention.
\end{abstract}




\section{Introduction}

At any moment, we are aware of only a small amount of the information provided in our environment. Attention is the nervous system's main mechanism to enhance processing of relevant information at the cost of irrelevant information. In the visual system, paying attention to a particular location in space increases perceptual sensitivity, accuracy, and spatial resolution and speeds up reaction times near the attentional focus (e.g. Posner et al., 1980; Hawkins et al., 1990; Yeshurun and Carrasco, 1998; Carrasco et al., 2002) while perceptually suppressing unattended stimuli (O'Regan et al., 1999).

Physiologically, attention strengthens the representation of attended aspects of the visual scene along all levels of the visual hierarchy by modulating responses of those neurons that are involved in processing these aspects (see Treue, 2003; Serences and Yantis, 2006, for reviews). Spatial attention selectively modulates firing rates of neurons with receptive fields overlapping the attended region in visual space, meaning those neurons which are tuned for the attended location (Tootell et al., 1998; Brefcynski and DeYoe, 1999).

While earlier studies have suggested that attention increases not only the sensitivity but also the selectivity of individual neurons for features like stimulus orientation or motion direction (Haenny and Schiller, 1988; Spitzer et al., 1988), more recent research has shown that attention modulates orientation and direction tuning curves in a multiplicative fashion without changing the tuning width (McAdams and Maunsell, 1999; Treue and Martinez-Trujillo, 1999; MartinezTrujillo and Treue, 2004). In the spatial domain, however, attentional effects can appear to be non-multiplicative even on the single neuron level: a few recent experiments have found in several visual areas that receptive field profiles shift towards an attended location (V4: Connor et al., 1996; Connor et al., 1997; LIP: Ben Hamed et al., 2002; MT: Womelsdorf et al., 2006) and receptive field area shrinks when attention is shifted into the receptive field (MT: Womelsdorf et al., 2006). Even though multiplicative modulations at hierarchically lower stages may underlie these changes, they are effectively non-multiplicative in nature and do change the selectivity of individual neurons by shifting and sharpening their spatial tuning curves.

Receptive fields in many visual areas have a complex substructure and consist not only of an excitatory receptive field center (the classical receptive field) but often have a surrounding region (the non-classical receptive field), where stimuli are thought not to drive the cell by themselves but modulate responses to a central stimulus. We have studied attentional effects on receptive fields in the motion processing area MT, where antagonistic surrounds are common that act inhibitory 
when stimulated with the cell's preferred direction (Tanaka et al., 1986; Lui et al., 2007). The exact proportion of MT cells which have such a surround varies from $50 \%$ (Perge et al., 2005) to $79 \%$ (Raiguel et al., 1995; Lui et al., 2007). Surrounds in area MT extend widely beyond the CRF radius by a factor ranging from 3-4 (Raiguel et al., 1995) to 7-10 (Allman et al., 1985; Tanaka et al., 1986) in different studies. Nearly half of MT surrounds have been suggested to be asymmetrically concentrated on one side of the CRF rather than being arranged circularly around it (Xiao et al., 1995; but see Tanaka et al., 1986).

Recently, attention has been found to change the suppressive versus integrative influence of receptive field surrounds in area $\mathrm{V} 1$, which is up to date the only direct evidence for an attentional impact on non-classical receptive field regions (Roberts et al., 2007; see also Ito and Gilbert, 1999, for an indirect measure of attentional effects on surround facilitation in V1). In extrastriate areas, it is currently not known if attention acts on receptive field surrounds, but recent studies show that in area MT as well as in V1, surround influences are not fixed but modifiable by stimulus properties and perceptual context and may even switch from inhibition to facilitation (V1: Kapadia et al., 1999; Sceniak et al., 1999; MT: Pack et al., 2005; Huang et al., 2007).

These recent findings suggest that attention is likely to modulate non-classical receptive field regions as well in area MT. The major effect of attention on excitatory receptive field regions in area MT is a shift in spatial position rather than a change in spatial summation. Here, we study in area MT how inhibitory surrounds are modulated by spatial attention. Specifically, we test if inhibitory surrounds shift towards an attended stimulus by mapping classical and nonclassical receptive fields under different attentional conditions with high resolution. Additionally, we provide new insights into attentional modulation of receptive field sizes and summation properties in area MT.

\section{Materials and methods}

\section{Monkey training and surgery}

Two male rhesus monkeys (Macaca mulatta) were trained to perform a visual spatial attention task. Following standard operant conditioning procedures, we used fluid reward as positive reinforcement for each correct trial in training and recording sessions. Animals were implanted with a custom made orthopedic implant to prevent head movements during training and recording, and a recording chamber (Crist Instruments, Hagerstown, MD) on top of a craniotomy over the left (monkey D) or the right (monkey $\mathrm{T}$ ) parietal lobe (monkey D : $6.5 \mathrm{~mm}$ posterior / $13 \mathrm{~mm}$ lateral, tilted backwards by 12 deg; monkey T: $9.2 \mathrm{~mm}$ posterior / 13.1 lateral, $6 \mathrm{deg}$ ). For monkey D, chamber positions were based on anatomical MRI 
scans. Surgeries were performed aseptically under isoflurane anesthesia using standard techniques. All procedures were approved by the animal ethics committee of the district government of Braunschweig, Lower Saxony, Germany.

\section{Apparatus}

Experiments were performed in a dimly lit cabin. Monkeys sat in a custom made primate chair at a distance of $57 \mathrm{~cm}$ from a computer monitor on which visual stimuli were presented. The monitor covered $48 \times 30 \mathrm{deg}$ of visual angle at a resolution of 40 pixel / deg. Refresh rate was $75 \mathrm{~Hz}$. Monkeys started a trial by touching a lever and responded by releasing the lever. Stimulus presentation, reward giving and collection of behavioral as well as electrophysiological data was controlled by custom software developed in-house and run on an Apple Macintosh computer.

\section{Electrophysiological recordings}

We recorded from 102 cells. Single unit action potentials were recorded extracellularly with either a single tungsten electrode (FHC Inc., Bowdoinham, ME) or a 5 channel system (Mini Matrix, Thomas Recording, Giessen, Germany). The dura mater was penetrated with sharp guide tubes so that electrodes could be inserted into the brain by a hydraulic micropositioner (single electrode; David Kopf Instruments, Tujunga, CA) or a rubber tube drive ( 5 channel system). Impedances ranged from 1 - $8 \mathrm{M} \Omega$.

Action potentials were recorded and sorted online using the Plexon data acquisition system (Plexon Inc., Dallas, TX). Data were filtered (frequency range $150 \mathrm{~Hz}-5 \mathrm{kHz}$ ) and amplified (gain range 1000 - 32000), single unit waveforms were isolated by window discrimination.

Area MT was identified by its anatomical position, the high proportion of direction selective cells, and the typical size-eccentricity relationship of receptive fields (mean eccentricity $8.3 \mathrm{deg} \pm 0.3 \mathrm{deg}$ S.E.M.; mean diameter $9 \mathrm{deg} \pm 0.3 \mathrm{deg}$ S.E.M. (measured in the fixation condition, see below); mean direction selectivity index (response preferred - response $\left._{\text {anti-preferred }}\right) /\left(\right.$ response $_{\text {preferred }}+$ response $\left._{\text {anti-preferred }}\right)=0.9$ \pm 0.02 S.E.M. for the 81 cells for which tuning data were available offline).

Eye positions were monitored using a video-based eye tracking system (ET-49, Thomas Recording, Giessen, Germany). Eye positions were recorded at $230 \mathrm{~Hz}$, digitized and stored at $200 \mathrm{~Hz}$. Fixation was controlled during the recordings to stay within a window of 1 deg radius around the fixation point (see below for details). 


\section{Experimental procedure}

After isolating a single unit, its cRF was identified by its response to a stationary random dot pattern (RDP) that was manually swept across the screen. To characterize speed and direction selectivity of the cell, the monkeys performed a task at the fixation point $(0.3 \times 0.3 \mathrm{deg}$, white, luminance contrast: $64.6 \%$ of full contrast at a background luminance of $0.02 \mathrm{~cd} / \mathrm{m}^{2}$ ) while a moving RDP (full contrast) was presented at the center of the estimated cRF. The task was the detection of a luminance change of the fixation point (luminance contrast after change: $53.4 \%$ ). The size of the RDP was matched to the cRF size. Individual dot size was $0.1 \times 0.1 \mathrm{deg}$ at a dot density of $10 \mathrm{dots} / \mathrm{deg}^{2}$. Motion speeds and directions were randomly drawn in intervals of $827 \mathrm{~ms}$ from 8 speeds logarithmically spaced between 0.5 and $64 \mathrm{deg} / \mathrm{s}$ and 12 evenly spaced directions between 0 (= upwards) and $330 \mathrm{deg}$. Responses to the individual speed direction combinations were defined as mean firing rates in an interval of $80-800$ ms after onset of the specific combination, and direction tuning curves were fit online with a circular Gaussian at each speed level. For the following experiments, a speed level was chosen at which there was a clear direction tuning, the direction yielding the highest response was defined as preferred direction and the opposite direction (180 deg apart) as anti-preferred. When recording several units on different channels, we used a direction and speed combination that activated all units as strongly as possible.

Before the main experiment was started, we mapped the receptive field by presenting a brief RDP (the probe, full contrast, $187 \mathrm{~ms}$ presentations separated by $27 \mathrm{~ms}$ ) moving in the preferred direction at several positions across the estimated receptive field. While the monkey performed a fixation task (detection of a luminance contrast change of the fixation point from $64.6 \%$ to $43.7 \%$ ), the probe was presented in a random sequence at $\sim 80$ positions on a rectangular grid (probe grid) centered on the estimated cRF. The size of the probe and the probe grid as well as the number of probe positions was adjusted to each cell. The probe grid spanned a circular or elliptical region with a radius $\sim 3$ times the estimated $\mathrm{cRF}$ radius. Individual dots of the probe were $0.1 \times 0.1$ deg wide at a density of 8 dots / $\mathrm{deg}^{2}$. We monitored online if the peak response was approximately in the middle of the probe grid. If it was, the main experiment was started using the same probe grid, otherwise, the probe grid was adjusted and the process was repeated.

\section{Attention task}

For the main experiment, the monkeys were trained to attend to one of two moving RDPs (target and distractor) placed at equal eccentricity inside or near the estimated $\mathrm{CRF}$ borders. The trial started when the monkey fixated a yellow fixation spot ( $0.25 \times 0.25 \mathrm{deg}$, luminance contrast: $92.7 \%)$ and held the lever. A stationary RDP (the cue) was presented for $440 \mathrm{~ms}$ at the later target location. After a delay of $133 \mathrm{~ms}$, target and distractor appeared (luminance contrast: $46.5 \%$ ), moving in 


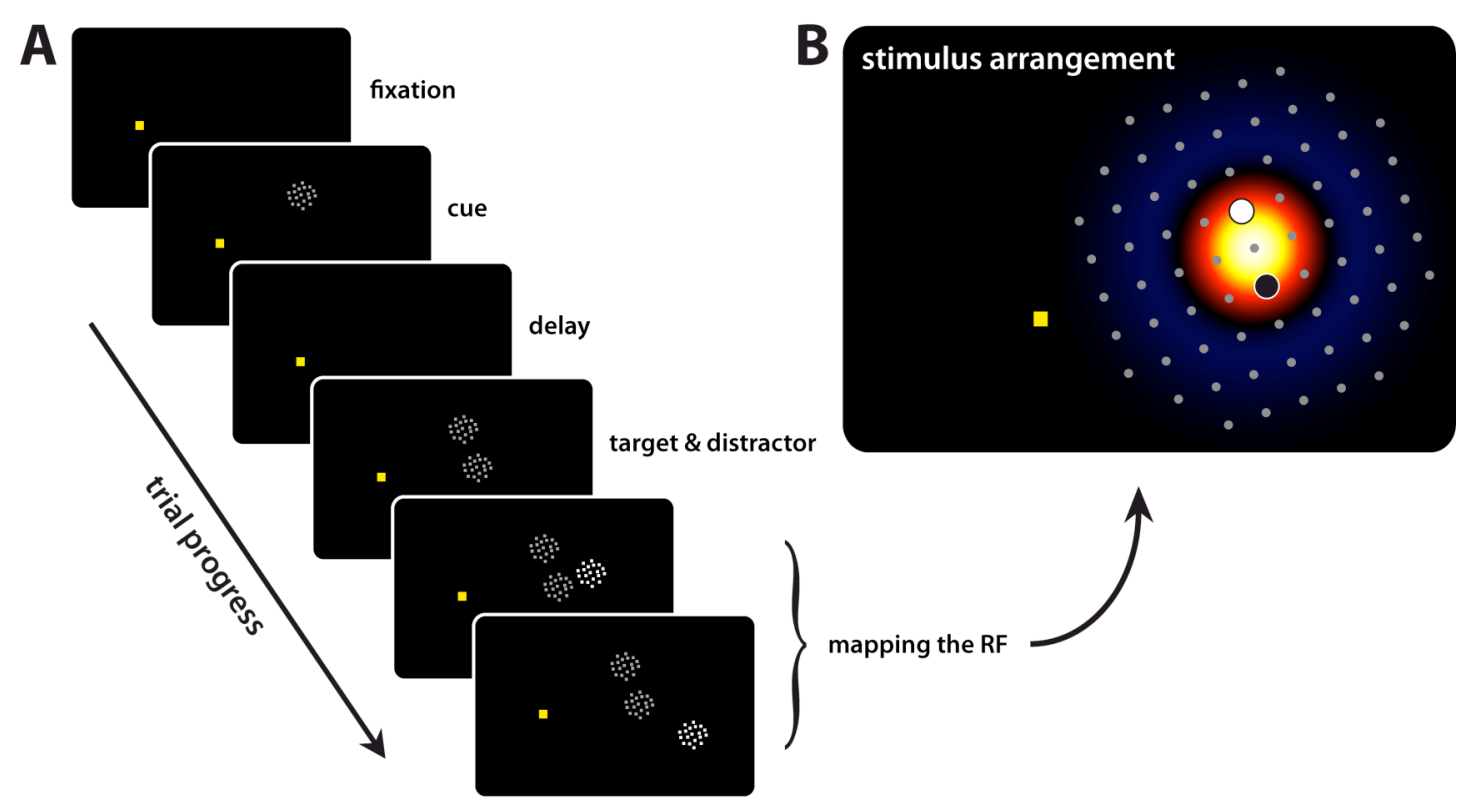

Figure 1: Attention task and stimulus arrangement. A: The trial started with fixation of the yellow fixation point. A stationary RDP, shown for $440 \mathrm{~ms}$, cued the later target position. After a delay of $133 \mathrm{~ms}$, target and distractor RDPs appeared, moving in the anti-preferred direction. After another $173 \mathrm{~ms}$, the mapping of the receptive field with the probe started. The probe RDP, moving in the preferred direction, was presented in random order at $\sim 80$ positions for $187 \mathrm{~ms}$ each, separated by $27 \mathrm{~ms}$. The monkey was rewarded for detecting a brief $(133 \mathrm{~ms})$ direction change of the target, which could occur between 253 and $6000 \mathrm{~ms}$ after target and distractor onset. B: Target (black circle filled white) and distractor (white circle filled black) were presented in or besides the estimated receptive field center at equal eccentricity from the fixation point. The probe grid (light grey dots) spanned the receptive field center (red/yellow) and surround (blue) and was arranged so that either one or two probe positions fell onto the receptive field center, between the target and distractor RDPs. Drawings are not to scale.

the anti-preferred direction. After another delay of $173 \mathrm{~ms}$, a sequence of probe presentations started. The probe, a full contrast RDP moving in the preferred direction, was presented in random order at $\sim 80$ positions on the same probe grid used for the initial mapping, the positions which overlapped target and distractor positions were skipped. Probes were presented for $187 \mathrm{~ms}$, separated by $27 \mathrm{~ms}$. Both target and distractor could change their direction of motion briefly $(133 \mathrm{~ms})$ by an angle of $\sim 35 \mathrm{deg}$. Times of the direction changes were drawn independently for each stimulus from a flat distribution between 253 and $6000 \mathrm{~ms}$ after stimulus onset. The monkeys were rewarded immediately after they responded by lever release to the direction change of the target within a response time window of 150 - $650 \mathrm{~ms}$ after the direction change onset. If they reacted to the distractor change, did not respond within the response time window or broke fixation, the trial was aborted without reward. The baseline firing rate of the neuron in each attentional condition was measured by skipping a probe in the sequence, that is within a 187 ms period the target and distractor stimuli were present without the probe, and this period was randomly interleaved with the probe presentations and had the same presentation statistics. Figure 1 shows the trial sequence and stimulus arrangement. 
As a control, we mapped the receptive field while the monkeys performed the fixation task. After the monkeys started the trial, the fixation spot turned white (luminance contrast: $64.6 \%$ ). There was no cue presentation; two RDPs (both distractors) appeared $553 \mathrm{~ms}$ after trial start at the same locations as target and distractor in the attention task. Otherwise the trial timing followed the same schedule as in the attention task. The monkeys were rewarded for the detection of a change of the fixation point from white to light grey (luminance contrast: $43.7 \%$ ) between 253 and 6000 ms after stimulus onset. The changes of motion direction in the distractors had to be ignored.

\section{Data analysis}

All calculations were performed with custom scripts written in MATLAB (The MathWorks, Natick, MA); all statistical tests were done using either MATLAB (The MathWorks, Natick, MA) or SPSS (SPSS Inc., Chicago, IL). Unless specified otherwise, errors given throughout the results are standard errors of the mean, statistical tests between unrelated groups are Mann-Whitney $U$ tests, comparisons of related groups or tests of one sample are Wilcoxon signed rank tests, and correlations are Spearman rank correlations. We relied on non-parametric tests because some of the tested parameters did not seem to be normally distributed and contained outliers; and when comparing two unrelated groups of cells, in some cases the assumption of equal variances was not met. All significant results reported here were also significant using parametric procedures.

\section{Receptive field maps}

We analyzed neuronal data from hit trials only to ensure that attention was appropriately allocated to the cued location. We created receptive field maps for each task condition (two attention conditions and one fixation condition) from the mean firing rates 60 - $140 \mathrm{~ms}$ after probe onsets at each probe position. This time window was chosen to capture the strongest part of the excitatory / inhibitory response. Responses to probe positions which had been presented less than twice were excluded (mean probe presentation frequency across all positions, cells, and conditions was $\sim 21$ ). From each mean firing rate we subtracted the mean baseline firing rate measured in the respective task condition. Receptive field maps were computed by interpolating these response rates at each probe location with a cubic spline function using 20 points between the measured positions. For simplicity, we rotated all receptive field maps so that the fixation point was up, the target and distractor lay along the x-axis, and the midpoint between target and distractor was at the origin.

\section{Identifying excitatory and inhibitory receptive field regions}

Based on the receptive field maps, we analysed responses above and below baseline separately. For each, we defined a threshold of a quarter of the maximum excitatory / inhibitory modulation rather than the commonly used half-height 
criterion to better accomodate the rather flat response modulation of the surround. In addition to this height - based criterion, we applied a spatial coherence - based criterion by including only patches which contained at least two sampled positions to remove spurious patches due to statistical fluctuations in individual probe responses. 'Holes' within patches were treated in the same way. Two of the 102 cells were excluded from all further analysis because their probe responses in the receptive field center were so weak that they did not pass this criterion.

\section{Quantifying inhibitory surrounds}

For each cell and each condition, we tested if the median of responses outside the excitatory center as defined above was significantly below baseline in a one-tailed sign test (alpha adjusted for multiple comparisons (100 cells * 3 conditions): 0.00017; overall significance level: 0.05 ). We classified a cell as having an inhibitory surround if the median firing rate outside the center was significantly below baseline in at least one of the three conditions, and only such 'surround cells' were included in all analyses of surround modulations (58 cells).

\section{Quantifying shifts of receptive field centers and surrounds}

Excitatory and inhibitory receptive field regions were analyzed separately. For each, we summed all height values from the interpolated maps falling into the patches which defined the respective receptive field region. The resulting value is a measure for the center and surround volumes. Note that because many surrounds appeared larger than the area we could measure, surround volume will refer only to the volume included in the region spanned by the probe grid. We calculated the centroid of this volume along the interstimulus axis as that $x$-value in the rotated map that divided the volume into halves. We then calculated shifts of center and surround volume centroids between the two attention conditions, divided by the CRF diameter. Positive shift values indicate a shift towards the attentional focus, negative values a shift in the opposite direction. We tested if the mean shift values across cells were different from zero. We also correlated the shift magnitudes with the distance between the attention targets normalized to the CRF diameter.

\section{Difference map analysis}

Difference maps were calculated for the 58 suround cells by first subtracting the response rates for each probe position when attention was on the left target in the rotated map from the corresponding firing rates when attention was on the right target, and then interpolating using the same surface fit as for the original maps. This would yield difference values in the surround to be more positive on the left than on the right if the surround shifts with attention (see Results). We identified the relevant regions of the difference map as follows: we first excluded all values belonging to the $\mathrm{CRF}$ in either of the two conditions. For defining both cRFs, we used the same procedure as before but placed the threshold at $5 \%$ of the 
maximum excitatory modulation because the modulation of the cRF seemed to extend over a larger area than the cRFs themselves as they were originally defined. Then, for surrounds that were symmetric along the orthogonal axis, we limited the analyzed region at the top and bottom of the region corresponding to the two centers. For surrounds that were significantly asymmetric along this axis (rank sum test for each cell of the median probe response in the fixation condition in the upper versus lower half outside the center, alpha adjusted for 58 tests: 0.00088 , overall significance value: $0.05,2$ cells), we analyzed the (upper or lower) half exhibiting the stronger inhibition. We computed the average of the firing rate differences left and right of the origin of the map from the data points within the respective selected region. We then tested across cells if the average firing rate difference on the left was different from the average firing rate difference on the right.

\section{Receptive field area}

We defined the square root of the area of the excitatory receptive field regions as the receptive field diameter. In the few cases in which the cRF consisted of several patches, we used the sum of their areas for calculating the diameter. Because by visual inspection of the maps we observed that many of our surrounds seemed to extend beyond the region covered by the probe grid, we do not provide a quantitative analysis of surround areas here. In order to investigate how attention modulates cRF size, we computed for each cell the relative size change in each of the attention conditions with respect to the fixation condition ((Diameter attention Diameter $\left._{\text {fixation }}\right)$ / Diameter $_{\text {fixation; }}$; two size change values for each cell). Negative values indicate a shrinkage with attention, positive values an expansion. We divided the 200 attention-fixation pairs into those for which attention was directed into the cRF patch $(n=142)$ and those for which attention was directed to a location at the border of the CRF (= outside the quarter-height threshold; $n=58$ ). We tested if each group mean was significantly different from zero size change and if the group means were significantly different from each other, and we also determined the correlation between the size change and the distance of the attentional target from the $\mathrm{cRF}$ area centroid, normalized to the receptive field radius.

\section{Analysis of baseline, peak response and inhibition strength}

We compared the baseline firing rate and the amplitude of the receptive field (= the maximum response after baseline subtraction) for all 100 cells in both attention conditions with the same measures in the fixation condition. For the 58 surround cells, we also compared the absolute minimum firing rate outside of the cRF and the depth of the surround (= the minimum response after baseline subtraction) between the attention and fixation conditions. To find out if attentional modulation of any of these parameters was related to the exact location of the attentional focus inside or at the border of the cRF, we calculated the same tests after 
dividing the attention - fixation pairs into cases where the attentional target was inside the $\mathrm{CRF}$ and cases where attention was at the border of the $\mathrm{CRF}$, and we calculated correlations between each of these parameters and the distance of the attentional target from the cRF center. We also directly compared the parameters between the two groups.

Because baseline changes, receptive field size changes and the distance between receptive field center and attentional focus might be all inter-related, we were interested if the correlation between size change and distance was caused by a correlation of the baseline change with this distance. Therefore we calculated a partial correlation between size change and distance, controlling for the effect of the baseline change. Since the partial correlation relies on the parametric (Pearson) correlation, we removed outliers: we iteratively excluded cases which deviated from the mean by more than 4 standard deviations with respect to either baseline change, size change, or distance, until no cases remained which deviated by more than 4 standard deviations. By this procedure, 6 cases were excluded.

\section{Eye Position Analysis}

All analyses of receptive field parameters were done over periods in which the monkey maintained fixation within the 1 deg circular fixation window. Still, systematic differences of the eye position within the fixation window could cause a corresponding shift of retinotopic receptive fields. We analyzed eye positions over the same time periods from which the neuronal data were taken. We rotated eye positions so that they were aligned with the rotated receptive field maps, and then tested the difference in mean eye position projected onto the interstimulus axis, and the difference in mean eye position along the orthogonal axis along the center of the stimulus grid and the fixation point, between the three task conditions by using a one-way repeated measures ANOVA ( 1 * 3 task conditions) and pairwise tests on the estimated marginal means. By this procedure, we analyzed eye positions along the same dimensions along which we analyzed receptive field shifts.

\section{Behavioral performance}

As a measure of the monkeys' performance, mean hit rates and mean reaction times were averaged across cells. We compared performance between the attention and fixation conditions. 


\section{Results}

Our study is based on 100 single neurons recorded in area MT of two macaque monkeys (58 cells from monkey $\mathrm{D}$ and 42 cells from monkey $\mathrm{T}$ ), while they directed their attention to one or the other of two stimuli placed within or besides the CRF of the neuron. For each neuron, we analyzed shifts of the excitatory receptive field center and of the inhibitory surround as well as changes of receptive field size, baseline firing rates, peak responses and inhibition strength with attention.

\section{Shifts of receptive field center and surround}

For each cell we mapped the receptive field under two attentional conditions: attention was either on the left stimulus or on the right stimulus, while the sensory stimulation and mapping procedure were exactly the same. Figure 2 shows two example cells. Indicated in the maps are the outlines of the excitatory (white) and inhibitory (grey) receptive field regions over which volume centroids for center and surround were determined. Center and surround shifts were defined as the difference in position of the respective centroid between the two attentional conditions, normalized to the CRF diameter. Both cells show a shift of the center as well as the surround towards the attended stimulus.

Figure $3 \mathrm{~A}$ shows the distribution of normalized center shifts: On average, the receptive field center shifts by $10.1 \%( \pm 1 \%)$ of its diameter towards the attended stimulus. This shift is highly significant $(p<0.0005)$ across cells. The average absolute magnitude of the center shift is $0.9 \mathrm{deg}( \pm 0.1 \mathrm{deg})$; and the average center shift normalized to the distance between the targets (which corresponds to the shift of spatial attention between the conditions, the 'attentional shift') is $13.8 \%$ $( \pm 1.2 \%)$.

The shift of the inhibitory surround was analyzed for the cells which were classified as surround cells $(n=58)$. For some cells, surround strength strongly varied under different conditions. 13 cells completely lost their inhibitory surround in one of the conditions even when inhibition was strong in the other condition. In figure 3B, the distribution of normalized surround shift values is plotted for the remaining 45 cells for which an inhibitory surround is present in both attentional conditions. They show a significant shift of the surround towards the attentional target which is on average $20.2 \%( \pm 7.7 \%)$ of the cRF diameter $(p=0.022)$. The average absolute magnitude of the surround shift is $1.6 \mathrm{deg}( \pm 0.7 \mathrm{deg})$; the average surround shift normalized to the attentional shift is $23.7 \%( \pm 11.1 \%)$. The surround shift is $14 \%$ ( \pm $6.8 \%)$ of the CRF diameter and remains significant $(p=0.031)$ when averaged over all 74 cells for which any inhibitory patches reached threshold in both conditions, including cells which were not classified as surround cells. 

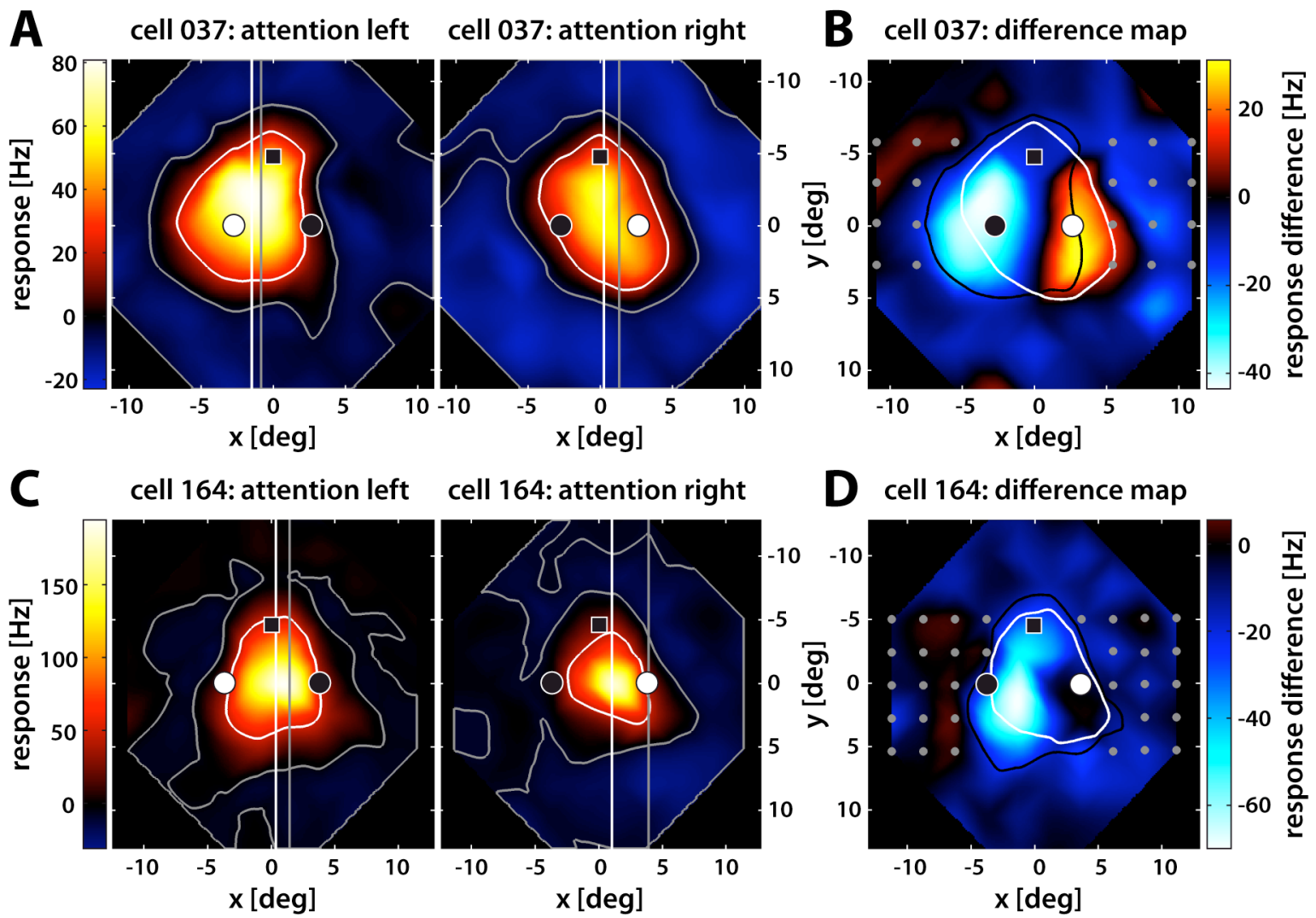

cell 164: attention right

D cell 164: difference map
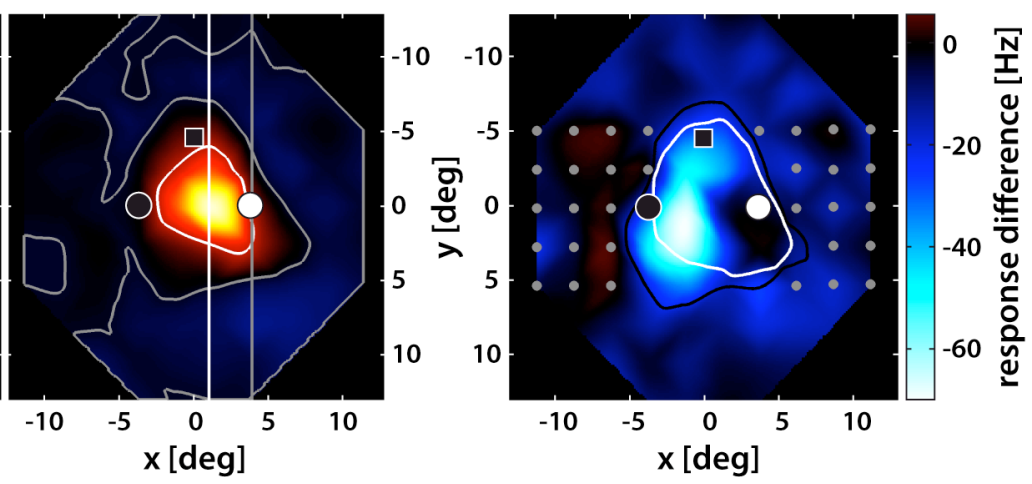

Figure 2: Center and surround shift for two example cells. A, C: Receptive field maps for two example cells when attention was either on the left or on the right target (black circle filled white). Maps were rotated for convenience so that the fixation point (white square filled black) was up. The contour lines mark the quarter-height level of excitatory / inhibitory modulation at which center and surround were cut for the centroid analysis (white: center, grey: surround). The vertical lines show the volume centroids (white: center, grey: surround) along the target-distractor axis, calculated over the outlined area. For both cells, center and surround profiles shift towards the attended stimulus. B, D: Difference maps for the same two cells were created by subtracting the probe responses with attention left from those with attention right. Regions of positive response differences (that is, stronger response with attention right) are shown in red/yellow, while negative response differences are shown in blue/cyan. Contour lines mark the $5 \%$ level of the cRF for attention left (black contour) and attention right (white contour). The grey dots show the probe positions that were used to calculate the mean response differences left and right of both centers (see Methods for details). For both cells, the response differences are more positive on the left than on the right of the $\mathrm{CRF}$, meaning that surround inhibition is weaker on the unattended side and stronger on the attended side.

Because some models of attentional CRF shifts predict a correlation of the shift magnitude with the distance of the attentional focus from the cRF center (Compte and Wang, 2006), we analyzed if the receptive field shifts in our population were related to the spacing of the targets, but we did not find such a correlation for neither center nor surround when shift and target spacing were normalized to the receptive field size ( $p=0.604$ and $p=0.169$ for center and surround, respectively).

\section{Dependence of center and surround measurements}

Typical receptive field center-surround structure can be well approximated by the difference of a peaked excitatory and a flatter inhibitory Gaussian, so that center and surround are spatially overlapping (DeAngelis et al., 1994; Raiguel et al., 
1995; Sceniak et al., 1999; Pack et al., 2005; Roberts et al., 2007). Assuming such a model, the above shift measures for center and surround are not independent of each other (Tzvetanov et al., 2005). Specifically, the shift of the center might induce an apparent shift of the surround by covering or uncovering some of the inhibitory regions. This apparent shift could be in the opposite or in the same direction, depending on the exact alignment or asymmetry between center and surround and would be indicative of a change of inhibitory regions in the 'effective' receptive field, but would not necessarily result from a change in those neuronal inputs that build the inhibitory surround. An apparent surround shift opposite to the center shift could have compensated a surround shift with attention, but we do observe a net shift towards the attentional focus. It is therefore important to clarify if the observed surround shift is a 'true' surround shift or a side effect of the center shift. One heuristic argument against the side effect interpretation is that the surround shift then should follow the center shift, but we find that it is in fact larger than the center shift. Another
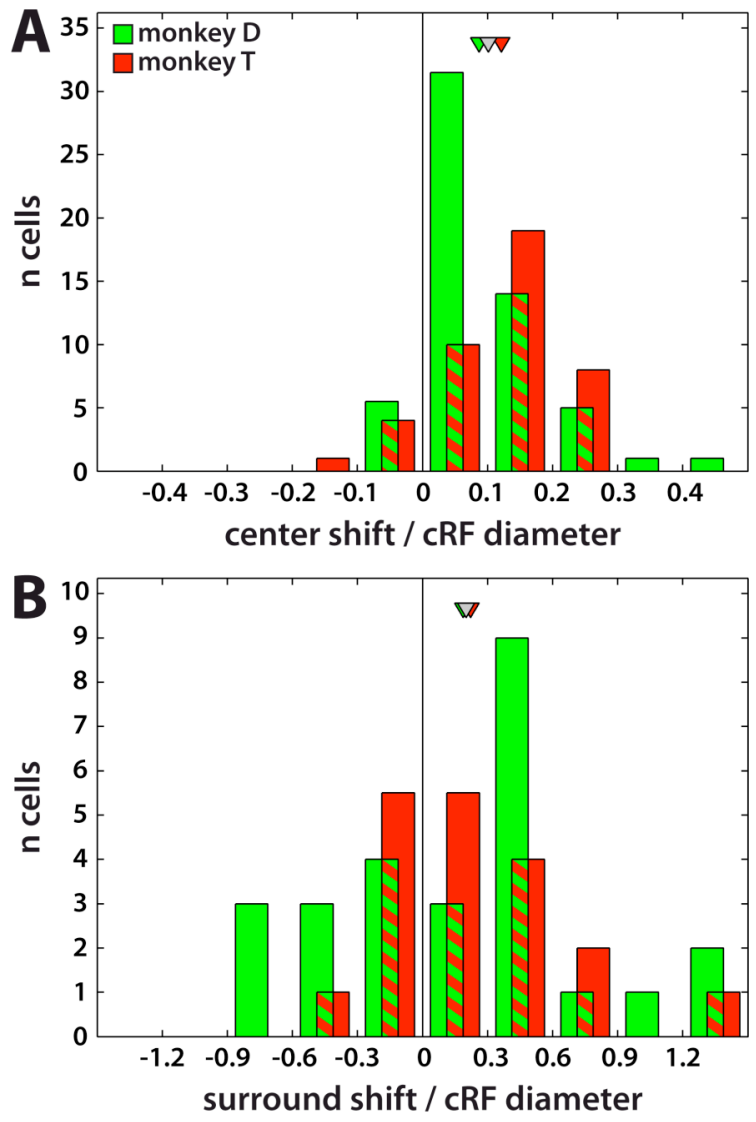

Figure 3: Distribution of center and surround shifts relative to the CRF diameter. A: The histogram shows a significant shift of the cRF center towards the attended stimulus by $10.1 \%$ ( $\pm 1 \%$ S.E.M., $p<0.0005, n=100$ ) of the $c R F$ diameter (monkey D (green): $8.7 \% \pm 1.2 \%, p<$ $0.0005, \mathrm{n}=58$; monkey $\mathrm{T}$ (red): $12.1 \%, \pm 1.5 \%$, $p<0.0005, n=42)$. B: The histogram shows a significant shift of the surround towards the attended stimulus by $20.2 \%( \pm 7.7 \%$ S.E.M., $p=$ $0.022, \mathrm{n}=45$ cells in the surround centroid analysis) of the cRF diameter (monkey D: not significant, $\mathrm{p}=0.182, \mathrm{n}=26$; monkey $\mathrm{T}: 22.3 \%$ $\pm 9.4, p=0.043, n=19)$. Triangles mark the mean shift magnitudes (grey: overall, green: monkey D, red: monkey T). argument is that if both shifts would depend on each other, then there should be a correlation between them: this is not the case $(p=0.5)$. Supplementary figure 1 plots the surround shift as a function of the center shift for the 45 cells for which the surround shift was analyzed.

In principle, the center shift could also be a side effect of the surround shift. We tested the group of 42 non-surround cells and found the cRF shift within this group to be highly significant (mean $10.1 \% \pm 1.2 \%$ of the CRF diameter, $p<0.0005$, data not shown), so the shift of the center is not contingent on the shift of the surround. To obtain a direct measure of the surround shift that is independent of the shift of 
A hypothetical difference map

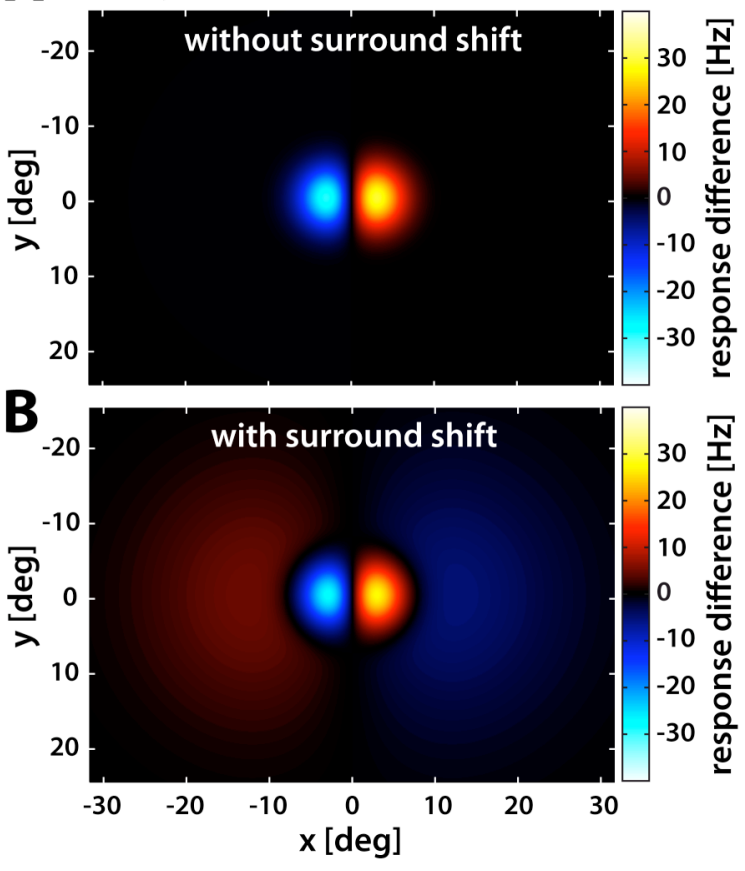

Figure 4: Hypothetical difference map. Hypothetical difference maps were created by subtracting two receptive field maps, each simulated by the difference of a narrow and peaked 2D-Gaussian (the receptive field center) and a spatially overlapping broad and flat 2DGaussian (the receptive field surround). A: Only the CRF is shifted between both conditions. Here, a leftward shift of the receptive field center was subtracted from a rightward shift, resulting in a peak of positive response differences on the right and a dip of negative response differences on the left. B: Attention additionally shifts the surround, resulting in an additional peak of positive response differences on the left and an additional dip of negative response differences on the right along the shift axis. See Supplementary Materials for further examples, formulas and choice of parameters. the center, we analyzed difference maps created by subtracting the receptive field maps of the two attention conditions. If the center shifts but the surround itself does not, the differences between firing rates outside the center should scatter around zero for all center-surround configurations. If there is a true surround shift, however, the map of firing rate differences between the two attention conditions should exhibit systematic changes. Figure 4 shows a hypothetical difference map for a cRF shift without surround shift $(A)$ and with surround shift (B). Assuming no difference in overall firing rate (amplitude) between the two attention conditions, the cRF shift appears as a peak close to the target of the first condition and a dip near the target of the subtracted condition. For a shift of a circularly symmetric surround as in $B$, there will be a bump of opposite sign at each side of the central bumps along the shift axis. If there is surround asymmetry along this axis, one of the bumps will be more pronounced than the other, while surround asymmetry along the orthogonal axis would shift the bumps along the y-axis, and a difference in overall firing rates would shift the whole map away from zero. Nevertheless, in all cases the difference between the respective regions in the map left and right of the $\mathrm{cRF}$ would remain qualitatively the same. Figure 4 assumes a symmetric surround and no amplitude modulation with attention; in the supplementary material we provide simulations of other configurations (supplementary figure 2).

Figures $2 \mathrm{~B}$ and $\mathrm{D}$ show the difference maps for the two example neurons. 'Attention left' has been subtracted from 'attention right'; the cRF outlines and attention targets of both conditions are marked in black and white, respectively. For both cells, the shift of the center is clearly visible from the two bumps overlapping these regions. In cell 164 (D), there is an additional scaling of the 


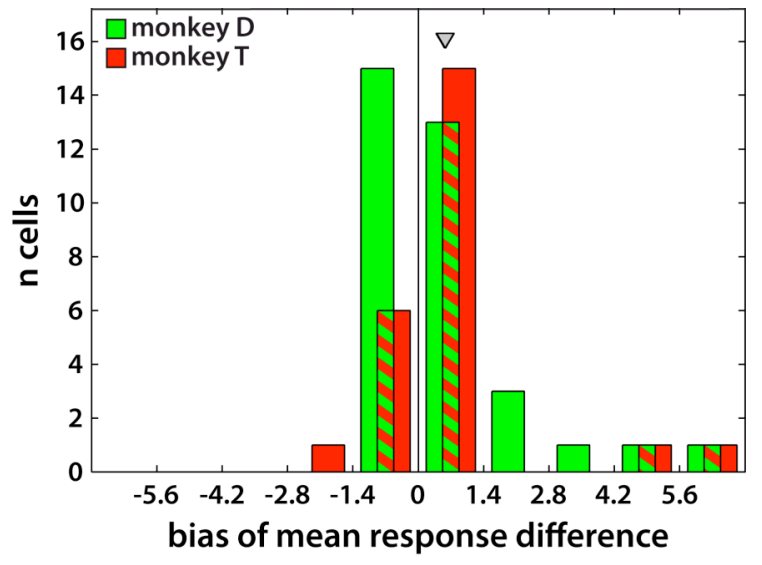

Figure 5: Distribution of the bias in mean response difference in the difference map analysis. The histogram shows a significant bias in mean response difference left and right of the $c R F$, so that the mean response difference is more positive on the left than on the right (mean $0.6 \mathrm{~Hz} \pm 0.2 \mathrm{~Hz}$ S.E.M., grey triangle, $p=0.013, n=58$ surround cells), corresponding to weaker surround inhibition on the unattended side and stronger surround inhibition on the attended side. Both monkeys analyzed separately show the same trend (monkey D (green): mean $0.6 \mathrm{~Hz}$ $\pm 0.3 \mathrm{~Hz}, \mathrm{p}=0.092, \mathrm{n}=34$; monkey $\mathrm{T}$ (red): mean $0.6 \mathrm{~Hz} \pm 0.3 \mathrm{~Hz}, \mathrm{p}=0.067, \mathrm{n}=24$ ).

receptive field between both conditions with responses being higher with 'attention left', therefore all difference values are below zero. For both cells, the difference map is more positive on the left than on the right of the central region, consistent with a true shift of the inhibitory surround. Figure 5 shows the distribution of differences in means left and right across the same 58 surround cells that were included in the volume shift analysis: The average difference is $0.6 \mathrm{~Hz}( \pm 0.2 \mathrm{~Hz})$ and is significant across cells $(p=0.013)$.

\section{Classical receptive field size changes}

In a previous study on attentional modulation of receptive fields (Womelsdorf et al., 2006), we tested the hypothesis that receptive fields contract around an attended stimulus and found a small but significant shrinkage with attention. Here, we measured receptive field sizes across a wider range of target position distances. Figure 6 shows receptive fields of two single cells in the fixation condition and when attention was directed into the CRF (A) or allocated to a spot at the border of the $\mathrm{cRF}(\mathrm{C})$. While the shrinkage of the $\mathrm{cRF}$ is clearly visible when attention is switched into the CRF, attention at the border of the cRF seems to expand rather than shrink the cRF. We found that across cells, the effects of attention on cRF size are different depending on the exact location of attention: For the group of 142 cases for which attention was switched into the cRF there is a highly significant mean shrinkage of CRF diameter by $4.7 \%( \pm 1.3 \%, p<0.0005)$. In contrast, for the group of 58 cases for which attention was directed to a location at the border of the $\mathrm{CRF}$, there is a significant growth of the cRF diameter by $14.2 \%$ $( \pm 4.6 \%, p=0.002)$. The average size change is also significantly different between the groups when directly compared with each other $(p<0.0005)$. Figures $6 \mathrm{~B}$ and $\mathrm{D}$ plot the distributions of size changes for both groups. Figure 7 plots the size change of each attention - fixation pair as a function of the normalized distance between the attention target and the cRF area centroid: The change in CRF size correlates significantly with this distance $(r=0.4, p<0.0005)$ and switches from shrinkage to expansion. 

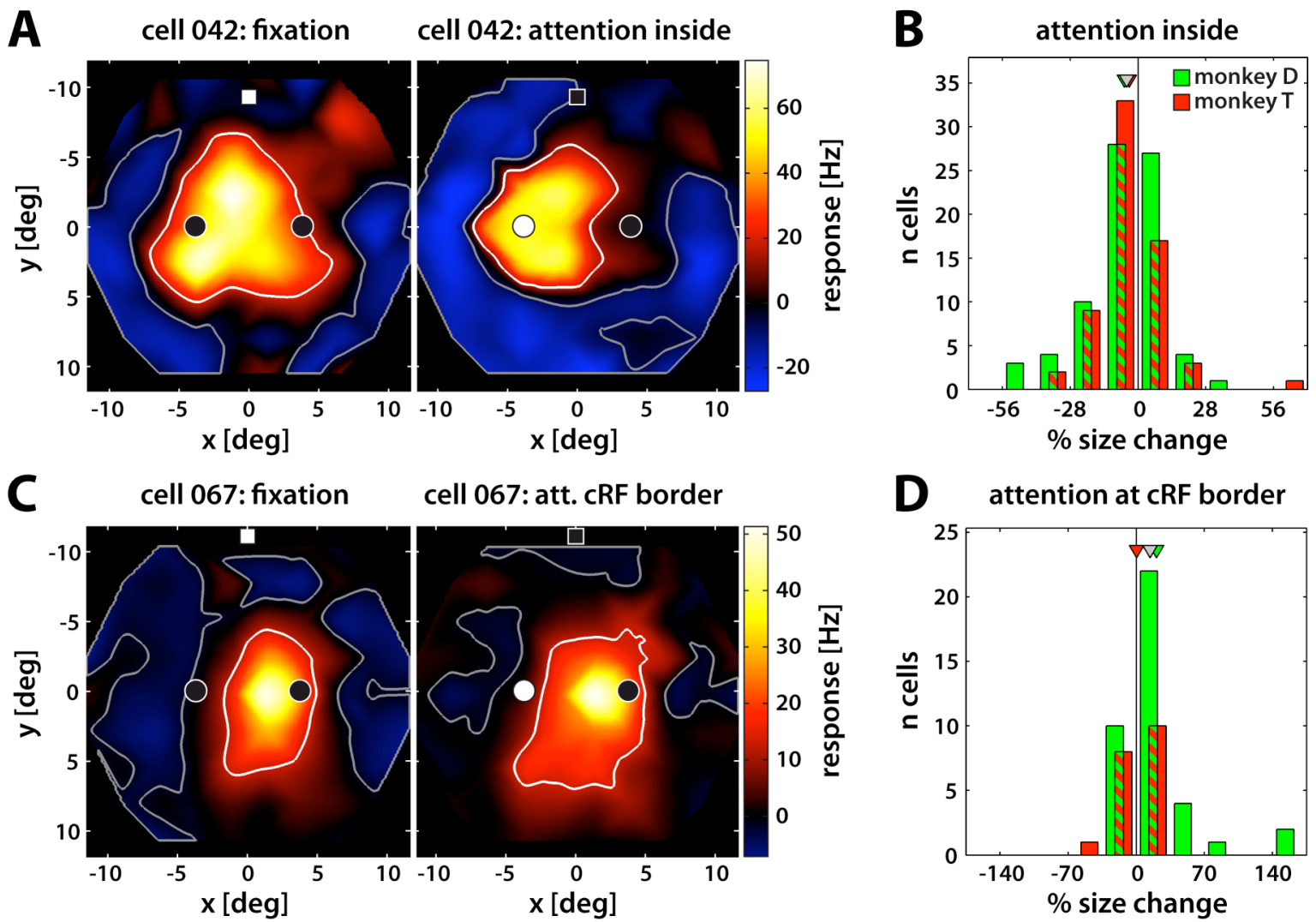

Figure 6: Size changes of the receptive field center with attention. A: Receptive field maps of an example cell when a task was done at the fixation point (left graph, white filled square) and when a position inside the cRF was attended (right graph, black circle filled white). The cRF area, outlined in white, is clearly reduced with attention inside the cRF. B plots the distribution of cRF size changes (in \% of the cRF diameter in the fixation condition) for all 142 cases in which attention was directed into the cRF. There is a significant shrinkage of $4.7 \%( \pm 1.3 \%$ S.E.M., $p<0.0005$; monkey D: $5.6 \%, \pm 1.8 \%, p=0.011, n=77$; monkey $\mathrm{T}: 3.6 \%, \pm 1.7 \%, \mathrm{p}=0.002, \mathrm{n}=65)$. In $\mathrm{C}$, receptive field maps of another example cell are compared for the fixation task (left graph) and attending to a spot at the border of the CRF (outside of the quarter-height defined excitatory region; right graph). Here, the cRF area grows with attention. D plots the distribution of cRF size changes for all 58 cases in which attention was directed to the border of the cRF. There is a significant expansion of $14.2 \%$ ( \pm 4.6\% S.E.M., $p=0.002$; monkey D: $20.8 \% \pm 6.4 \%, p<0.0005, n=39$; monkey T: not significant, $\mathrm{p}=0.717, \mathrm{n}=19)$.

\section{Baseline shifts and receptive field scaling}

Because we observed that for some cells, baseline firing rates, peak responses and surround strength varied quite strongly between conditions, we determined if these variations were related to the allocation of attention into or near the CRF. We analyzed changes in baseline firing rate, amplitude (the peak response relative to baseline), surround depth (the minimum response relativ to baseline), and the absolute minimum firing rate in the surround (without baseline subtraction).

Across all cells, the mean baseline firing rate was $8.4 \mathrm{~Hz}$ in the fixation condition, and was significantly enhanced with attention by on average $1.5 \mathrm{~Hz} \pm 0.4 \mathrm{~Hz}(\mathrm{p}=$ 0.006 ). The baseline change is highly significant for those attention - fixation pairs for which the attentional focus was inside the cRF $(2.3 \mathrm{~Hz} \pm 0.6 \mathrm{~Hz}, \mathrm{p}<0.0005, \mathrm{n}$ $=142$ ), but not for those pairs with attention directed to a location at the border of 
the $\operatorname{cRF}(p=0.123, n=58)$. The difference in baseline change between the groups is highly significant $(p<$ 0.0005), and we found a highly significant negative correlation of the baseline change with the distance of the attentional focus from the $\mathrm{cRF}$ center $(r=-0.33, p<0.0005)$.

Across cells, the mean cRF amplitude was $45.9 \mathrm{~Hz}$ in the fixation condition. Across all cells and also for the group of cases for which attention was oriented inside the $\mathrm{CRF}$, there was no effect of attention on the amplitude (all: $p=0.121$; 'attention inside': $p=$ 0.981 ), but for those cases for which attention was directed to the border of the CRF, we found a significant increase of CRF amplitude with attention $(3.8 \mathrm{~Hz} \pm 1.2 \mathrm{~Hz}, \mathrm{p}=0.004$, $\mathrm{n}=58$ ). The difference in amplitude change between the groups is significant $(p=0.014)$, and we found a weak but significant correlation of the

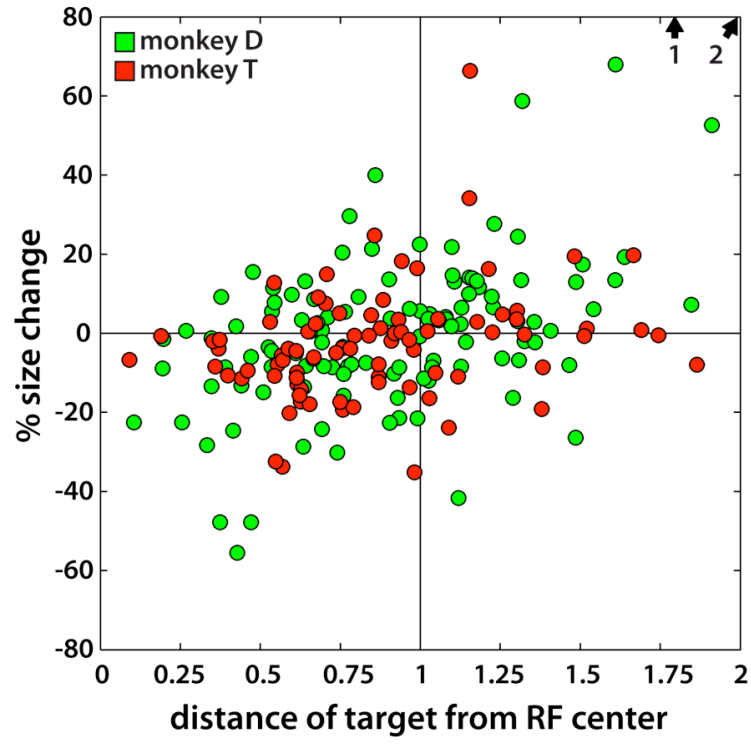

Figure 7: Receptive field size change as a function of the distance of the attentional target to the receptive field center. For the 200 attention-fixation pairs, the change in $\mathrm{CRF}$ diameter is plotted as a function of the distance between target and CRF center, which was normalized to the CRF radius so that a distance of 1 approximately marks the cutoff between attention inside and attention besides the cRF. Arrows mark data points that fall beyond the axis limits, numbers indicate how many data points are represented by each arrow. The correlation between size change and targetcenter distance is significant $(r=0.4, p<$ 0.0005 ; monkey $\mathrm{D}: \mathrm{r}=0.46, \mathrm{p}<0.0005, \mathrm{n}=$ 116; monkey $\mathrm{T}: \mathrm{r}=0.34, \mathrm{p}=0.002, \mathrm{n}=84$ ). amplitude change with the distance of the attentional focus from the cRF center $(r=0.163 ; p=0.021)$.

For the 58 surround cells, the mean surround depth was $5.8 \mathrm{~Hz}$ in the fixation condition. There was no overall effect of attention on the surround depth $(p=$ 0.937). However, when attention was inside the cRF, surround depth was not significantly changed but there is a trend for deeper surrounds with attention (mean depth change $1 \mathrm{~Hz} \pm 0.5 \mathrm{~Hz}, \mathrm{p}=0.054, \mathrm{n}=76$ ). In contrast to this, when attention was allocated to a target near the $\mathrm{cRF}$, surrounds became significantly flatter $(1.4 \mathrm{~Hz} \pm 0.7 \mathrm{~Hz}, p=0.004, \mathrm{n}=40)$. This difference between the groups is highly significant $(p=0.001)$, and there is a correlation $(r=0.35, p<0.0005)$ between the change in surround depth and the distance of the attentional focus from the cRF center.

Because surround depth was measured relative to baseline, a deepening or flattening of surrounds does not necessarily result from a change of the strength of inhibitory responses, but might instead be a consequence of systematic baseline changes. Indeed, we found that the absolute minimum firing rate (without baseline 
subtraction) was higher in the attention conditions than in the fixation condition (mean difference $0.8 \mathrm{~Hz} \pm 0.3 \mathrm{~Hz}, \mathrm{p}<0.0005$, at an average minimum rate of 1.8 $\mathrm{Hz}$ in the fixation condition). This increase is significant for those attention fixation pairs for which the attentional focus was inside the CRF as well as for those cases with attention at the border of the cRF (attention inside: $1 \mathrm{~Hz} \pm 0.4 \mathrm{~Hz}$, $p<0.0005, n=76$; attention near: $0.5 \mathrm{~Hz} \pm 0.1 \mathrm{~Hz}, \mathrm{p}=0.001, \mathrm{n}=40$; the difference between the groups is not significant although there is a trend, $p=$ 0.091), so that the change in relative surround depth must be related to the change in baseline firing rate.

We were wondering if the differential changes in baseline firing rate depending on the location of the attentional target influenced our analysis of receptive field shrinkage and expansion, because receptive field sizes were determined from baseline-corrected maps. We indeed found a clear correlation between baseline change and size change (rank correlation $r=-0.61, p<0.0005, n=200$; Pearson correlation $r=-0.46, p<0.0005, n=194$ after outlier correction (see Methods)), but we also found a partial correlation between size change and distance of the attentional target from the CRF center, controlling for baseline changes $(r=0.29, p$ $<0.0005, \mathrm{n}=194$ after outlier correction (see Methods)). This means that the variation of cRF size changes with distance from the attentional focus is related to the baseline changes, but is not completely explained by it.

As mentioned above, for some cells the baseline, cRF amplitude and surround depth seemed highly variable between the attentional conditions; but across cells, the differences in baseline, amplitude, and depth were not significantly different from zero, so this could not influence our shift measures in any systematic way (baseline: $p=0.365$; amplitude: $p=0.864$; depth: $p=0.306$ ).

\section{Eye positions}

Systematic differences of the monkeys' eye position within the fixation window could in principle cause a corresponding shift of the mapped receptive fields. We tested for a shift in eye position along the interstimulus axis across cells using a one-way repeated measures ANOVA $(1 * 3$ task conditions). There was no significant main effect on the eyeposition along the interstimulus axis $(p=0.162)$, but pairwise comparisons of estimated marginal means yielded a very small yet significant difference between eye position in the two attention conditions (mean difference $=0.02 \mathrm{deg}, p=0.014$ ). Furthermore, this difference was in the opposite direction as the observed receptive field shift, so it cannot explain the differences in receptive field position. In addition, we did not find a significant correlation between eye position difference along this axis and either center or surround shifts between the attentional conditions (here, the absolute shift values were used; center: $p=0.419$; surround: $p=0.892$ ), and we also did not find a correlation between eye position difference and the result of the difference map analysis ( $p=$ 
0.241). There was, however, a significant difference of the eye position along the orthogonal axis between the fixation task and both of the attentional conditions: eye positions were closer to the RDPs when the monkeys were involved in the attention task (main effect of condition: $p=0.008$, pairwise comparisons of estimated marginal means between fixation and attention condition 1 and 2: mean $=0.03, p=0.078$ and mean $=0.03, p=0.013$ ). This should not influence any comparisons between the two attention conditions, though. Supplementary figure 3 shows the distribution of eye positions in the three task conditions for the example cells from figure 2 and the distribution of mean eye positions across all cells.

\section{Behavioral performance}

Mean hit rates over all trials were $87.8 \% \pm 0.01 \%$ in the fixation task (monkey D: $86.3 \% \pm 0.01 \%$, monkey $\mathrm{T}: 89.9 \% \pm 0.01 \%$ ) and $69.0 \% \pm 0.01 \%$ in the attention task (D: $71.4 \% \pm 0.01 \%, \mathrm{~T}: 65.7 \% \pm 0.02 \%$ ). We also analyzed the hit rates without considering fixation errors to get a better estimate how many of the errors were due to not allocating attention correctly to the target, that is reacting too early or missing the target change. This hit rate was on average $97.8 \% \pm 0.003 \%$ in the fixation task (D: $98.8 \% \pm 0.003 \%, \mathrm{~T}: 96.4 \% \pm 0.005 \%$ ) and $82.8 \% \pm 0.01 \%$ in the attention task (D: $86.3 \% \pm 0.01 \%, \mathrm{~T}: 78.1 \% \pm 0.02 \%)$. Mean reaction times were $361 \mathrm{~ms} \pm 4 \mathrm{~ms}$ in the fixation task (D: $381 \mathrm{~ms} \pm 5 \mathrm{~ms}, \mathrm{~T}: 333 \mathrm{~ms} \pm 6$ ) and $412 \mathrm{~ms} \pm$ $5 \mathrm{~ms}$ in the attention task (D: $450 \mathrm{~ms} \pm 4 \mathrm{~ms}, \mathrm{~T}: 359 \mathrm{~ms} \pm 6 \mathrm{~ms}$ ). Both monkeys were faster ( $p<0.0005$ for both monkeys together and separate) and more accurate ( $p<0.0005$ for both monkeys together and separate) in the fixation task, but they were also doing the more demanding attention task with a high performance. Supplementary figure 4 graphs these results.

\section{Discussion}

Switching spatial attention between two stimuli inside or near an MT neuron's classical receptive field shifts the center of the classical receptive field as well as the inhibitory surround profile towards the attended stimulus. Center shifts were on average $10.1 \%$ of the receptive field diameter, while the surround profile shifted by twice this distance, $20.2 \%$. Systematic changes of surround strength on the attended and unattended side of the receptive field indicate that attention modulates the surround itself, independent of the attentional effects on the classical receptive field. In addition, we find that excitatory receptive field sizes are reduced when attention is directed into the receptive field by $4.7 \%$, but attention expands receptive fields by $14.2 \%$ if the attentional target is outside of the classical receptive field. Absolute surround strength can be highly variable for the same cell when measured under different attentional conditions: surrounds tend to be deepened if attention is directed into the cRF but are flattened if attention is 
allocated to a target at the border between CRF and surround. This is related to changes in baseline firing rate, which is enhanced for attention targets inside the CRF but not for attention targets outside the cRF. Baseline modulation is correlated to receptive field size changes, but cannot explain the total variation of the shrinkage / expansion effect with distance from the attentional focus.

By shifting excitatory and inhibitory regions towards an attended location, attention changes the profile of a single neuron's spatial tuning function in a nonmultiplicative fashion. Because on average the shift of the surround is larger than that of the center, the effect is not just a position shift of the spatial tuning curve as a whole. Instead, excitatory gain and surround suppression both become stronger near the attended location, meaning that effectively the spatial configuration of the receptive field complex is changed.

\section{Possible mechanisms of a surround shift}

Receptive fields are commonly thought to increase in size and complexity as one moves up the visual hierarchy by accumulating more and more inputs from lowerlevel areas. Attention may differentially modulate the gain of lower-level receptive fields (e.g. in V1) representing the attended and unattended locations and thereby cause a biased weighting of inputs to receptive fields in higher-level areas like MT, which would lead to a shift of excitatory receptive field profiles in the higherlevel area towards the attended position (Maunsell and McAdams, 1999; McAdams and Maunsell, 2001). Such a feedforward model has been shown to be able to account for CRF shifts towards the attentional focus given a relatively large spatial spread of attention (Compte and Wang, 2006; T. Womelsdorf, T. Tzvetanov, K. Anton-Erxleben, S. Treue, unpublished observations).

In contrast to excitatory receptive field regions which are mainly built from feedforward connections, there are several possibilities how antagonistic surrounds could arise in area MT, which are not necessarily mutually exclusive. One possibility is that the center-surround structure of feedforward input is transferred to MT receptive fields (Tanaka et al., 1986). On the other hand, higher visual areas with large receptive fields could provide inhibitory feedback to create antagonistic surrounds in area MT (Tanaka et al., 1986), a hypothesis supported by the finding that response latencies are longer in the surround than in the center (Allman et al., 1985; Perge et al. 2005). Alternatively, surrounds could be created within area MT, either by horizontal connections (Allman et al., 1985), or by inhibitory connections from especially large receptive fields found in layer 5 (Raiguel et al., 1995). Consistent with the latter view, the deeper layers (4 - 6) in area MT contain most of the cells without inhibitory surround (Tanaka et al., 1986; Raiguel et al., 1995; Lui et al., 2007). 
Given our paradigm for mapping the inhibitory surround, it is possible that we stimulate mainly feedforward components of the surround. Our paradigm differs from earlier approaches in two respects: we do not present an optimal stimulus inside the cRF while mapping the surround, and we stimulate the surround using brief probes which might not be optimal for a full-blown surround suppression known to lag behind the excitatory response by between $16 \mathrm{~ms}$ (Perge et al., 2005) and $40 \mathrm{~ms}$ (Allman et al., 1985). Still, we find significant suppressive responses below baseline and systematic shifts of surround volume - so our method captures the surround.

Assuming that MT surrounds do have a strong feedforward component in which the center-surround structure of $\mathrm{V} 1$ receptive fields is contained, a similar mechanism could shift excitatory and inhibitory receptive field regions: Attentional gain modulation

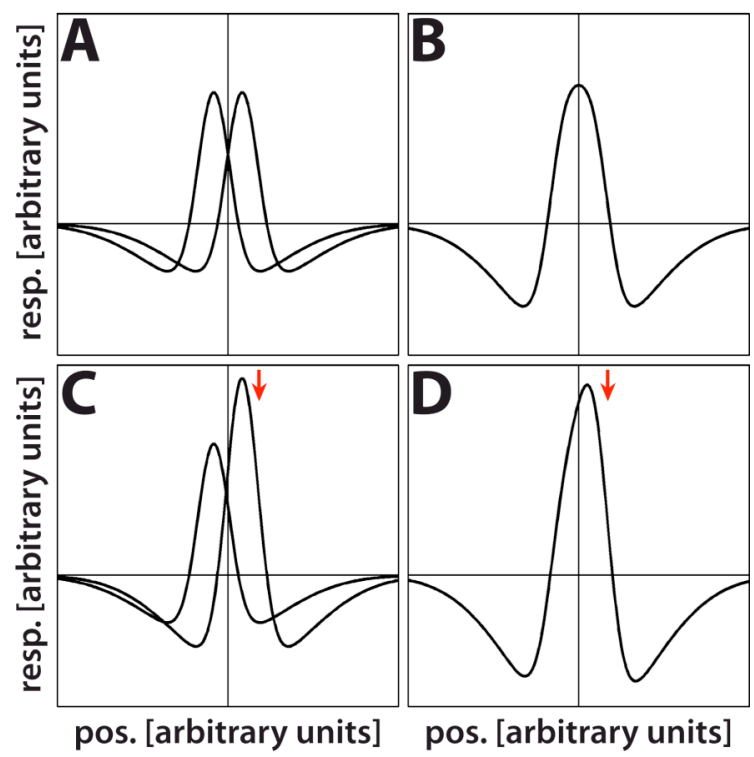

Figure 8: A mechanism for a feedforward surround shift. A: Spatial tuning profiles (response as a function of position) of two V1 receptive fields with inhibitory surrounds are represented by the difference of two Gaussians. $B$ : Their additive combination results in a broader spatial tuning curve, with inhibitory surround, representing the receptive field of an MT neuron. In $C$ and $D$, the same receptive fields are shown with attention selectively increasing the gain of that $\mathrm{V} 1$ receptive field closer to the attentional focus (marked by the red arrow): Multiplicative gain modulation of one of the $\mathrm{V} 1$ receptive fields $(\mathrm{C})$ shifts the peak of the MT receptive field towards the direction of spatial attention, and at the same time increases surround strength on the same side (D).

could selectively strengthen the surrounds of those $\mathrm{V} 1$ receptive fields which overlap the attended location and so deepen the surround of an MT neuron selectively on the attended side of its receptive field - this would shift the inhibitory surround profile in MT towards the attentional focus (Figure 8). Recent research however suggests that such an attentional strengthening of surround inhibition in V1 occurs only for parafoveal receptive fields, while surround inhibition seems to be weakened by attention at eccentricities more similar to those sampled in our study (Roberts et al., 2007). To which extent characteristics of task and stimulus, for example the relatively low contrast range used by Roberts and colleagues for the peripheral receptive field sample, influence this finding needs further investigation: it is conceivable that at low contrast, which is known to enhance spatial summation rather than segmentation in V1 (Kapadia et al., 1999; Sceniak et al., 1999), attention strengthens summation even more, but at high contrast, which favours segmentation, attention might have the opposite effect. At least a component of MT surrounds seems to be formed by feedback or intra-areal connections coming from larger receptive fields found either in higher visual areas 
or particular layers within area MT (see above). How those large receptive fields could provide the spatial specificity required to induce a surround shift is not clear.

\section{Functional implications of a surround shift}

A large amount of research has established antagonistic surrounds as a general organizing principle throughout the visual system and current research discovers more and more of their complex spatiotemporal characteristics. Although antagonistic surrounds are associated with a variety of different perceptual functions, the direct link is not completely understood. Generally, center-surround organization has been implicated in figure-ground segregation (Lamme, 1995; Zipser et al., 1996; Yazdanbakhsh and Livingstone, 2006), pop-out (Kastner et al., 1997), the detection of line ends and curvature (Julesz, 1981; Dobbins et al., 1987; Dobbins et al., 1989), and perceptual constancy (Allman et al., 1985). Motion sensitive surrounds in particular have been suggested to be important for image segmentation based on motion as well as perceiving relative motion of objects and self-motion in the environment (Allman et al., 1985; Bradley and Andersen, 1998; Born et al., 2000). The asymmetric center-surround structures found in area MT may be useful for extracting shape from motion (Buracas \& Albright, 1996; Liu and Van Hulle, 1998).

In the present experiment, the attentional targets were almost always in excitatory regions of the receptive field or at the border of excitatory and inhibitory regions, but nearly never within the deep portions of the surround. Therefore, a shift as we observe would typically bring the relevant stimulus into the CRF or closer to the cRF center, while the surround inhibition is strengthened just beyond the attended location. Such a shift might function to increase the attended stimulus' influence on the cell's response but also to actively suppress stimulation in its close vicinity and reduce influences of distractors, thus enhancing spatial resolution. A strengthening of center-surround antagonism at the attended location would also improve the perception of local-to-local motion contrast.

\section{Integrative and suppressive receptive field properties are variable with attention}

Attentional effects on receptive field surrounds that have been reported previously are quite different from the shift effect we describe here: In V1, attention changes integrative and suppressive receptive field characteristics (Roberts et al., 2007); an effect that has also been found to occurr with variations in stimulus contrast (Kapadia et al., 1999; Sceniak et al., 1999). We observed that surround strength is variable within the same cell also in our sample of MT neurons. These findings challenge the concept that the presence of an inhibitory surround is a fixed property of a cell. 
We found a trend of surround depth to vary systematically with the distance of the receptive field from the attentional focus, so that surrounds tended to become deeper when attention was within the excitatory part of the receptive field but became flatter when attention was focused at the border of the receptive field. This seemed to be related to shifts in baseline firing rates, as if the cell shifted its reference line and thereby were able to switch between segmentation and integration.

Additionally, we found systematic changes in the size of the excitatory receptive field center: when attention was allocated inside the CRF borders, the CRF contracted around the attended stimulus, but when attention was directed outside, the CRF grew towards the attended stimulus. Such a switch from CRF shrinkage to CRF expansion is predicted by the feedforward model of attentional modulation proposed by Compte and Wang (2006). Both, an increase in CRF size as well as a flattening of surround inhibition would lead to spatial integration of sensory inputs over a wider area, while the shrinkage of the CRF together with a stronger surround inhibition would favor segmentation of the scene. An expansion of nearby receptive fields towards the focus of attention could be a mechanism to allocate more neuronal resources to the attended stimulus, so that its properties might be encoded more accurately and its representation would be strengthened. On the other hand, receptive fields which already include the attended stimulus might receive stronger surround inhibition in order to suppress distractive signals from outside. The shift of the receptive field profile would contribute in both cases to bring the attended stimulus closer to excitatory receptive field regions while suppressing nearby locations more strongly.

In summary, we provide evidence that excitatory receptive field centers and inhibitory receptive field surrounds shift with attention in a way that changes the spatial configuration of the receptive field. This effect combines with a general attentional up- and down- modulation of integrative versus suppressive surround influences. We think that the strengthening of center-surround structure near the attended location may be an important mechanism by which attention improves spatial resolution. 


\section{References}

Allman J, Miezin F, McGuiness E (1985) Direction- and velocity-specific responses from beyond the classical receptive field in the middle temporal visual area (MT). Perception 14: 105-126.

Ben Hamed S, Duhamel J-R, Bremmer F, Graf W (2002) Visual receptive field modulation in the lateral intraparietal area during attentive fixation and free gaze. Cereb Cortex 12(3): 234-245.

Brefczynski JA, DeYoe EA (1999) A physiological correlate of the 'spotlight' of visual attention. Nature 2(4): 370-374.

Bradley DC, Andersen RA (1998). Center-surround antagonism based on disparity in primate area MT. J Neurosci 18(18): 7552-7565.

Born RT, Groh JM, Zhao R, Lukasewycz SJ (2000) Segregation of object and background motion in visual area MT: Effects of microstimulation on eye movements. Neuron 26(3): 725-734.

Buracas GT, Albright TD (1996) Contribution of area MT to perception of threedimensional shape: a computational study. Vision Res 36(6): 869-887.

Carrasco M, Williams PE, Yeshurun Y (2002) Covert attention increases spatial resolution with or without masks: Support for signal enhancement. Journal of Vision 2: 467-479.

Compte A, Wang X-J (2006) Tuning curve shift by attention modulation in cortical neurons: a computational study. Cereb Cortex 16: 761-778.

Connor CE, Gallant JL, Preddie DC, Van Essen DC (1996) Responses in area V4 depend on the spatial relationship between stimulus and attention. J Neurophysiol 75(3): 1306-1308.

Connor CE, Preddie DC, Gallant JL, Van Essen DC (1997) Spatial attention effects in macaque area V4. J Neurosci 17(9): 3201-3214.

DeAngelis GC, Freeman RD, Ohzawa I (1994) Length and width tuning of neurons in the cat's primary visual cortex. J Neurophysiol 71(1): 347-374.

Dobbins A, Zucker SW, Cynader MS (1987) Endstopped neurons in the visual cortex as a substrate for calculating curvature. Nature 329: 438-441.

Dobbins A, Zucker SW, Cynader MS (1989) Endstopping and curvature. Vision Res 29(10): 1371-1387.

Haenny PE, Schiller PH (1988) State dependent activity in monkey visual cortex. I. Single cell activity in V1 and V4 on visual tasks. Exp Brain Res 69(2): 225244.

Hawkins HL, Hillyard SA, Luck SJ, Mouloua M, Downing C, Woodward DP (1990) Visual attention modulates signal detectability. J Exp Psychol Hum Percept Perform 16(4): 802-811.

Huang X, Albright TD, Stoner GR (2007) Adaptive surround modulation in cortical area MT. Neuron 53: 761-770. 
Ito M, Gilbert CD (1999) Attention modulates contextual influences in the primary visual cortex of alert monkeys. Neuron 22: 593-604.

Julesz B (1981) Textons, the elements of texture perception, and their interactions. Nature 290: 91-97.

Kapadia MK, Westheimer G, \& Gilbert CD (1999) Dynamics of spatial summation in primary visual cortex of alert monkeys. Proc Natl Acad Sci USA 96(21): 12073-12078.

Kastner S, Nothdurft H-C, Pigarev IN (1997) Neuronal correlates of pop-out in cat striate cortex. Vision Res 37(4): 371-376.

Lamme VAF (1995) The neurophysiology of figure-ground segregation in primary visual cortex. J Neurosci 15(2): 1605-1615.

Liu L, Van Hulle MM (1998) Modeling the surround of MT cells and their selectivity for surface orientation in depth specified by motion. Neural Comput 10: 295312.

Lui LL, Bourne JA, Rosa MGP (2007) Spatial summation, end inhibition and side inhibition in the middle temporal visual area (MT). J Neurophysiol 97: 11351148.

Martinez-Trujillo JC, Treue S (2004) Feature-based attention increases the selectivity of population responses in primate visual cortex. Curr Biol 14: 744-751.

McAdams C, Maunsell JHR (1999) Effects of attention on orientation-tuning functions of single neurons in macaque cortical area V4. J Neurosci 19(1): 431-441.

Maunsell, JHR, McAdams, CJ (1999) Effects of Attention on Neuronal Response Properties in Visual Cerebral Cortex. In: The New Cognitive Neurosciences (Gazzaniga MS, ed), pp. 315-324. MIT Press, Cambridge, MA.

Maunsell, JHR, McAdams, CJ (2001) Effects of attention on the responsiveness and selectivity of individual neurons in visual cerebral cortex. In: Visual Attention and Cortical Circuits (Braun J, Koch C, Davis JL, eds), pp 103119. MIT Press, Cambridge MA.

O'Regan JK, Rensink RA, Clark JJ (1999) Change-blindness as a result of 'mudsplashes'. Nature 398: 34.

Pack CC, Hunter JN, Born RT (2005) Contrast dependence of suppressive influences in cortical area MT of alert macaque. J Neurophysiol 93: 18091815.

Perge JA, Borghuis BG, Bours RJE, Lankheet MJM, van Wezel RJA (2005) Dynamics of directional selectivity in $\mathrm{MT}$ receptive field centre and surround. Eur J Neurosci 22: 2049-2058.

Posner MI, Snyder CR, Davidson BJ (1980) Attention and the detection of signals. J Exp Psychol 109(2): 160-174.

Raiguel S, Van Hulle MM, Xiao D-K, Marcar VL, Orban GA (1995) Shape and spatial distribution of receptive fields and antagonistic motion surrounds in 
the middle temporal area (V5) of the macaque. Eur J Neurosci. 7(10): 20642083.

Roberts M, Delicato LS, Herrero J, Gieselmann MA, Thiele A (2007) Attention alters spatial integration in macaque $\mathrm{V} 1$ in an eccentricity-dependent manner. Nat Neurosci 10(11): 1483-1491.

Sceniak MP, Ringach DL, Hawken MJ, Shapley R (1999) Contrast's effect on spatial summation by macaque V1 neurons. Nat Neurosci 2(8): 733-739.

Serences JT, Yantis S (2006) Selective visual attention and perceptual coherence. Trends Cogn Sci 10(1): 38-44.

Spitzer H, Desimone R, Moran J (1988) Increased attention enhances both behavioral and neuronal performance. Science 240(4850): 338-340.

Tanaka K, Hikosaka K, Saito H, Yukie M, Fukada Y, Iwai E (1986) Analysis of local and wide-field movements in the superior temporal visual areas of the macaque monkey. J Neurosci 6(1): 134-144.

Tootell RBH, Hadjikhani N, Hall EK, Marrett S, Vanduffel W, Vaughan JT, Dale AM (1998) The retinotopy of visual spatial attention. Neuron 21: 1409-1422.

Treue S (2003) Visual attention: the where, what, how and why of saliency. Curr Opin Neurobiol 13: 428-432.

Treue S, Martinez-Trujillo JC (1999) Feature-based attention influences motion processing gain in macaque visual cortex. Nature 399: 575-579.

Tzvetanov T, Womelsdorf T, Treue S (2005) A general model of multiplicative attentional modulation of the receptive field structure. Computational and Systems Neuroscience (COSYNE) meeting, abstract 269.

Womelsdorf T, Anton-Erxleben K, Pieper F, Treue S (2006) Dynamic shifts of visual receptive fields in cortical area MT by spatial attention. Nat Neurosci 9(9): 1156-1160.

Xiao D-K, Raiguel S, Marcar V, Koenderink J, Orban GA (1995) Spatial heterogeneity of inhibitory surrounds in the middle temporal visual area. Proc Natl Acad Sci USA 92: 11303-11306.

Yazdanbakhsh A, Livingstone MS (2006) End stopping in V1 is sensitive to contrast. Nat Neurosci 9(5): 697-702.

Yeshurun Y, Carrasco M (1998) Attention improves or impairs visual performance by enhancing spatial resolution. Nature 396(6706): 72-75.

Zipser K, Lamme VAF, Schiller PH (1996) Contextual modulation in primary visual cortex. J Neurosci 16(22): 7376-7389. 
Supplementary Material for

\title{
ATTENTION MODULATES CENTER-SURROUND STRUCTURE IN AREA MT
}

\author{
K. Anton-Erxleben, V. M. Stephan, S. Treue
}

The supplementary material provides additional information about several aspects of our results:

We show

- the distribution of center shift magnitudes as a function of surround shift magnitudes

- the generality of the principles of the difference map analysis for different center-surround asymmetries and overall response changes between the two conditions

- the distribution of eye positions in the attention conditions and the distribution of eye position differences between the attention conditions

- the behavioral performance of the monkeys in the different task conditions

\section{Surround shift as a function of center shift}

Our data show that inhibitory surrounds shift towards the attentional focus. From the centroid analysis (see main text) it is not clear if this is a side effect of the shift of the classical receptive field (cRF) or if attention directly modulates the connections forming the inhibitory surround. If it was a side effect caused by the covering / uncovering of inhibitory regions due to the cRF shift, the surround shift should lag behind the cRF shift and be closely correlated to it. We did not find a significant correlation between $\mathrm{CRF}$ and surround shift, neither for the 45 cells in the volume centroid analysis ( $p=0.5$; supplementary figure 1 ), nor for the whole cell population ( $p=0.36$ ).

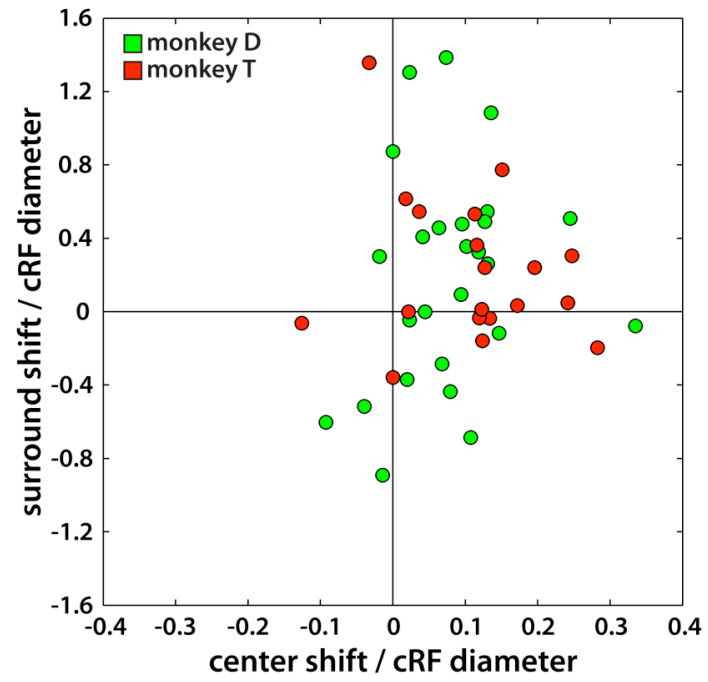

Supplementary figure 1: Surround shift as a function of the center shift. For each of the 45 cells included in the volume centroid analysis of the surround shift, the shift of the surround is plotted as a function of the center shift (green: monkey D, red: monkey $\mathrm{T})$. 

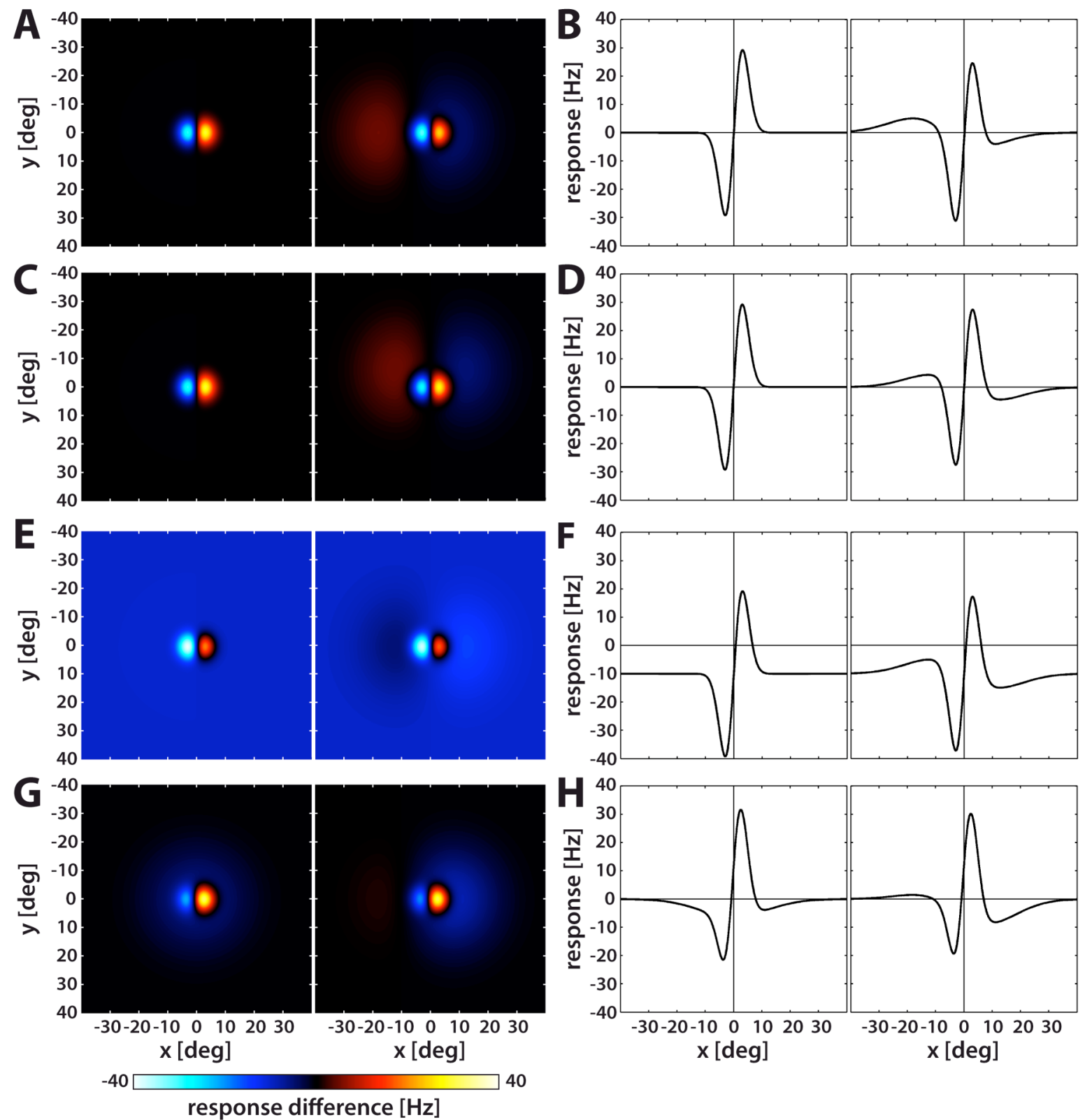

response difference [Hz]

\begin{abstract}
Supplementary figure 2: Hypothetical difference maps for different center-surround configurations. Hypothetical difference maps were created by subtracting two receptive field maps, each simulated by the difference of a narrow and peaked 2D-Gaussian (the center) and a spatially overlapping broad and flat 2D-Gaussian (the surround). Receptive field shifts between both conditions were along the horizontal; a leftward shifted receptive field map was subtracted from a rightward shifted receptive field map, so that the shift of the center is visible as a peak of positive response differences on the right and a dip of negative response differences on the left. In $A$ and $B$, the surround was asymmetric to the left of the center; in $C$ and $D$, the surround was asymmetric to the top of the figure; in $\mathrm{E}$ and $\mathrm{F}$, a change in overall response rate between both conditions was included additively; in $\mathrm{G}$ and $\mathrm{H}$, the response change was a multiplicative factor. $\mathrm{A}, \mathrm{C}, \mathrm{E}$, and $\mathrm{G}$ show the difference maps; $B, D, F$, and $H$ show the profile through the $x$-axis of the plots in $A, C, E$, and G. The left column of each figure shows the difference map / profile for a pure center shift, the right column shows the difference map / profile for a shift of center and surround. A pure center shift does not lead to differences in response change left and right outside the center (for additive or multiplicative scaling between the two conditions, E-H, the change affects locations in the surround, but in the same direction left and right). In contrast, when there is a surround shift, the response change on the left is always more positive than the response change on the right of the center.
\end{abstract}




\section{Difference maps for different center-surround configurations}

Assuming a difference-of-Gaussians model of the receptive field center-surround structure with spatially overlapping center and surround, attentional modulation of the surround itself can be distinguished from a side effect of the attentional modulation of the cRF by systematic changes in the difference maps of the two attentional conditions. A shift of the CRF alone is visible in the difference map by a peak of positive differences near the attended stimulus and a dip of negative differences near the unattended stimulus, both confined to the region overlapping both centers. If the surround shifts in the same direction as the center, however, the difference map shows two bumps within the surround, flanking the central peak and dip but of opposite sign (see main text, Results, and figure 4).

Supplementary figure 2 shows simulations of difference maps to demonstrate that the same principle holds for asymmetric center-surround organization as well as overall firing rate differences between the two conditions.

If the inhibitory surround is asymmetric along the interstimulus axis, the surround shift still leads to the same characteristic changes, but they are stronger on one side than on the other ( $A$ and $B$ ). If the inhibitory surround is asymmetric along the orthogonal axis, the region in which the difference map deviates from zero is displaced along this axis $(C$ and $D)$. If the overall firing rate relative to baseline is different between the conditions, the whole difference map is shifted up or down, depending on the direction of the change (down in $E$ and $F$ ). A similar result is obtained if one of the receptive fields is instead multiplied by a constant ( $G$ and $H$ ), but here the overall up- or down- shift fades out with distance from the center.

Receptive fields were simulated by the difference of two two-dimensional Gaussians:

$R F_{\text {att.left }}=A_{c t r} * \exp \left(-\frac{\left(x-\left(x m_{c t r}-\frac{s h i f t_{c t r}}{2}\right)\right)^{2}}{2 s x_{c t r}^{2}}-\frac{\left(y-y m_{c t r}\right)^{2}}{2 s y_{c t r}^{2}}\right)-A_{s u r} * \exp \left(-\frac{\left(x-\left(x m_{\text {sur }}-\frac{s h i f t_{\text {sur }}}{2}\right)\right)^{2}}{2 s x_{\text {sur }}^{2}}-\frac{\left(y-y m_{\text {sur }}\right)^{2}}{2 s y_{\text {sur }}^{2}}\right)$

$R F_{\text {att.right }}=A_{c t r} * \exp \left(-\frac{\left(x-\left(x m_{c t r}+\frac{s h i f t_{c t r}}{2}\right)\right)^{2}}{2 s x_{c t r}^{2}}-\frac{\left(y-y m_{c t r}\right)^{2}}{2 s y_{c t r}^{2}}\right)-A_{s u r} * \exp \left(-\frac{\left(x-\left(x m_{s u r}+\frac{s h i f t_{s u r}}{2}\right)\right)^{2}}{2 s x_{s u r}^{2}}-\frac{\left(y-y m_{\text {sur }}\right)^{2}}{2 s y_{\text {sur }}^{2}}\right)$

where $A_{c t r}$ is the amplitude, $x m_{c t r}$ and $y m_{c t r}$ are the centroid coordinates, $s x_{c t r}$ and 


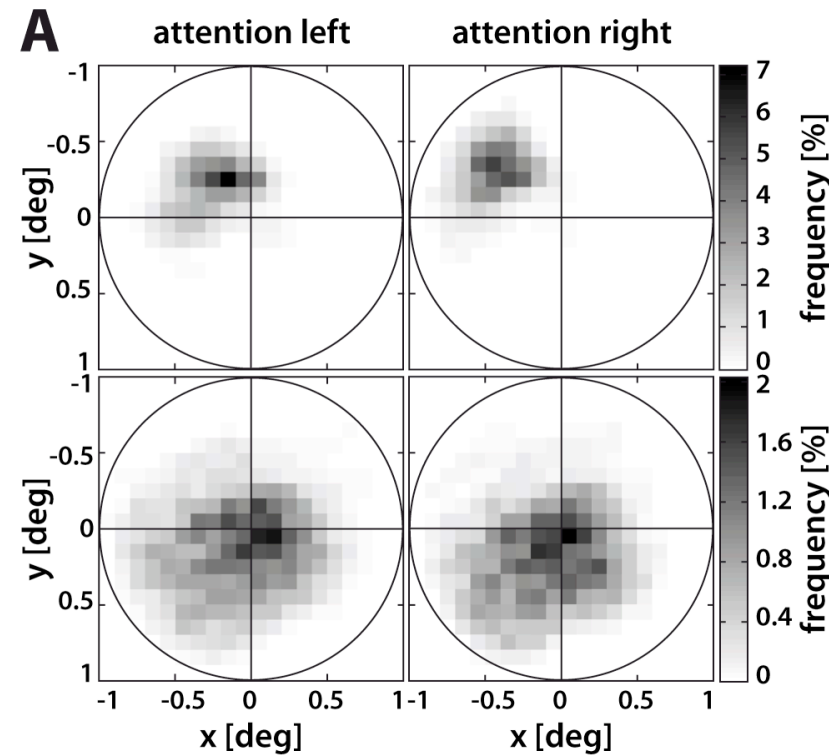

B

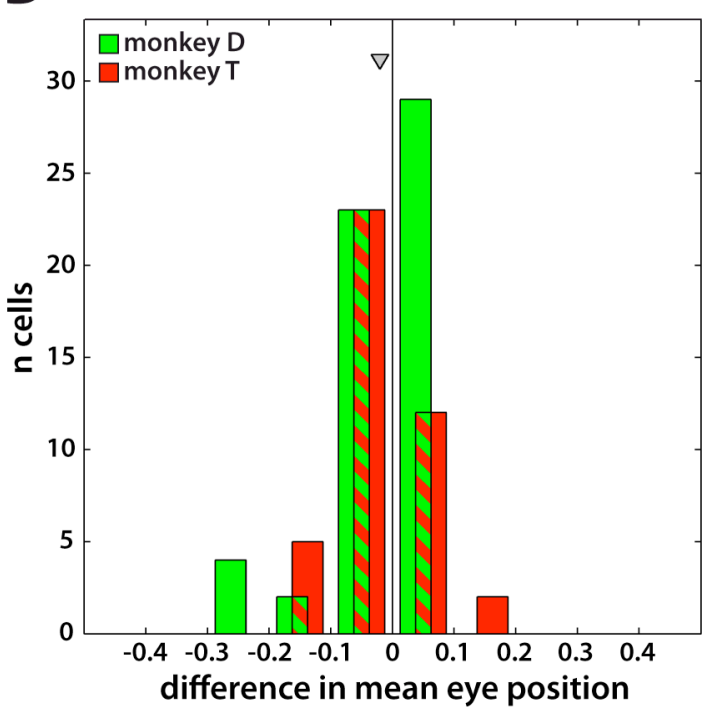

Supplementary figure 3: Eye positions. A: Distribution of eye positions relative to the fixation window for cells 037 (top row) and 164 (bottom row), the same example cells which are shown in figure 2. The alignment of the maps is the same as in figure 2, the cross indicates the fixation point and the circle marks the fixation window. B: Distribution of the difference in mean eye position between the attentional conditions along the axis between the attentional targets (green: monkey $\mathrm{D}$, red: monkey $\mathrm{T}$, triangle: mean across all cells).

$s y_{c t r}$ are the standard deviations of the Gaussian along $\mathrm{x}$ and $\mathrm{y}$, respectively, for the receptive field center. $A_{\text {sur }}, x m_{\text {sur }}, y m_{\text {sur }}, s x_{\text {sur }}$ and $s y_{\text {sur }}$ are the same parameters for the receptive field surround. Here, circular receptive fields were assumed; the basic principles also apply for elliptical shapes. The parameter values used for the receptive fields in figure 2 were: $A_{c t r}=75 \mathrm{~Hz}, x m_{c t r}=0 \mathrm{deg}, y m_{c t r}=0 \mathrm{deg}, s x_{c t r}=3$ deg, $s y_{\text {ctr }}=3 \mathrm{deg}, A_{\text {sur }}=25 \mathrm{~Hz}, x m_{\text {sur }}=0 \mathrm{deg}, y m_{\text {sur }}=0 \mathrm{deg}, s x_{\text {sur }}=12 \mathrm{deg}, s y_{\text {sur }}=12$ deg; asymmetries along $\mathrm{x}$ and y were implemented using $x m_{s u r}=-6$ or $y m_{s u r}=-6$, respectively. The shift of the receptive field center (shift $t_{c t r}$ ) was always 2 deg, the shift of the surround (shift $t_{\text {sur }}$ ) was either 0 deg or 4 deg.

Difference maps were created by:

Diff $=R F_{\text {att.right }}-R F_{\text {att.left }}$

in figure $2 A-D$;

Diff $=R F_{\text {att.right }}-\left(B+R F_{\text {att.left }}\right)$

with $B=10 \mathrm{~Hz}$ in figure $2 \mathrm{E}$ and $\mathrm{F}$;

Diff $=R F_{\text {att.right }}-\left(B * R F_{\text {att.left }}\right)$

with $B=0.75$ in figure $2 \mathrm{G}$ and $\mathrm{H}$;

\section{Eye positions}

Because receptive fields in area MT are retinotopic, i.e. they move with the eye, small differences between the conditions in mean eye position within the fixation window could have contributed to the observed receptive field shifts. We analyzed 

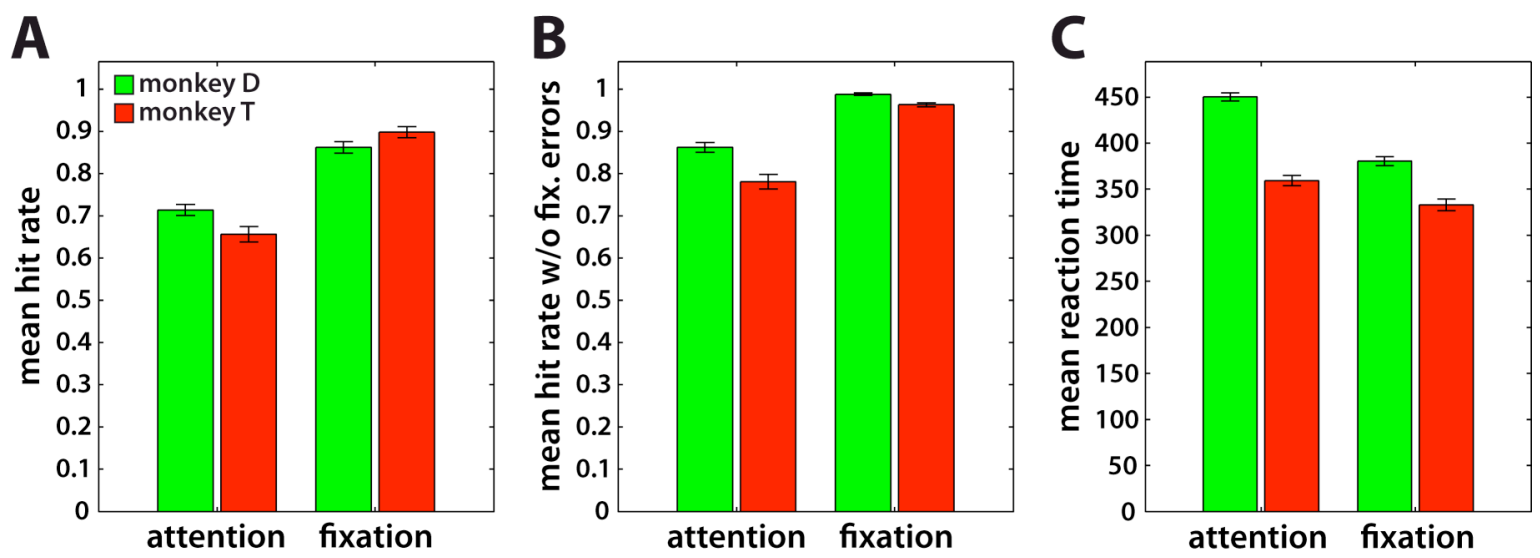

Supplementary figure 4: Behavioral performance. A: Mean hit rates in the attention and fixation task for monkey D (green) and monkey T (red); B: Mean hit rates not considering fixation breaks; C: Mean reaction times. Error bars are S.E.M.

mean eye position across cells and found a very small but significant deviation of $0.02 \mathrm{deg}$ in the opposite direction (away from the attended stimulus; $p=0.014$ ). Therefore, eye position differences cannot account for our results. Supplementary figure 3 shows the distribution of eye positions relative to the fixation point for the two attention conditions for the two example cells which illustrate the center and surround shifts in figure 2 (main text). We also did not find a significant correlation between eye position difference along the attentional shift axis and either center or surround shifts (here, the absolute shift values were used; $p=0.419$ and $p=0.892$ for center and surround, respectively), and we did not find a correlation between eye position difference and the result of the difference map analysis $(p=0.241)$.

\section{Behavioral performance}

We analyzed mean hit rates in the attention and the fixation task with and without considering fixation errors. While errors that result from missing the target change or reacting too early, possibly to the distractor change, indicate that the monkey did not correctly interpret the cue and did not direct his attention to the target, breaks of fixation are a different type of error not necessarily related to understanding the task. Both hit rates are very high, though. Hit rates as well as reaction times show that the fixation task was significantly easier for the monkeys ( $p<0.0005$ for hit rates and reaction times; supplementary figure 4 , see also main text, Results). 



\subsection{Receptive field shift and shrinkage in macaque area MT through attentional gain modulation}

This study shows that attentional modulations of receptive field profiles in area MT can be well described by a feedforward model in which lower-level inputs from attended and unattended locations are differentially scaled in a multiplicative fashion. In addition, the model allows us to draw conclusions about the spatial spread of the attentional focus. 



\title{
RECEPTIVE FIELD SHIFT AND SHRINKAGE IN MACAQUE
}

\section{AREA MT THROUGH ATTENTIONAL GAIN MODULATION}

\author{
Thilo Womelsdorf ${ }^{1,2,}$, , Tzvetomir Tzvetanov ${ }^{2,{ }^{*},}$, Katharina Anton-Erxleben ${ }^{2}$, \\ Stefan Treue ${ }^{2,3}$
}

${ }^{1}$ F.C. Donders Centre for Cognitive Neuroimaging, Radboud University, Kapittelweg 29, 6525 EN Nijmegen, The Netherlands ${ }^{2}$ Cognitive Neuroscience Laboratory, German Primate Center, Kellnerweg 4, 37077 Göttingen, Germany ${ }^{3}$

Dept. of Biology, Göttingen University, Gosslerstrasse 14, 37077 Göttingen, Germany

${ }^{*}$ authors that contributed equally to the presented work

\begin{abstract}
Selective attention is the top-down mechanism to allocate neuronal processing resources to the most relevant subset of the information provided by an organism's sensors. Attentional selection of a spatial location modulates the spatial tuning characteristics, i.e. the receptive fields of neurons in macaque visual cortex. These tuning changes include a shift of receptive field centers towards the focus of attention and a narrowing of the receptive field when the attentional focus is directed into the receptive field. Here, we report that when attention is directed into versus out of receptive fields of MT neurons the magnitude of the shift of the spatial tuning functions is positively correlated with a narrowing of spatial tuning around the attentional focus. By developing and applying a general attentional gain model we show that these nonmultiplicative attentional modulations of basic neuronal tuning characteristics could be a direct consequence of a spatially distributed multiplicative interaction of a bell shaped attentional spotlight with the spatially fined-grained sensory inputs of MT neurons. Additionally, the model lets us estimate the spatial spread of the attentional top-down signal impinging on visual cortex. Consistent with psychophysical reports the estimated size of the 'spotlight of attention' indicates a coarse spatial resolution of attention. These results illustrate how spatially specific non-multiplicative attentional changes of neuronal tuning functions can be the result of multiplicative gain modulation affecting sensory neurons in a widely distributed region in cortical space.
\end{abstract}




\section{Introduction}

Selective attention modulates sensory responses of neurons throughout the visual cortex (Reynolds and Chelazzi, 2004; Maunsell and Treue, 2006). Attention has been shown to modulate neuronal responses based on spatial and feature selection (Treue and Martinez-Trujillo, 1999; McAdams and Maunsell, 2000; Martinez-Trujillo and Treue, 2004; Bichot et al., 2005), it affects neuronal contrast response functions, possibly increasing apparent stimulus contrast (Reynolds et al., 2000; Martinez-Trujillo and Treue, 2002; Carrasco et al, 2004; Williford and Maunsell, 2006), scales the temporal integration functions of sensory inputs (Cook and Maunsell, 2004), and increases response selectivity of the neuronal population response (Martinez-Trujillo and Treue, 2004). These diverse effects of attention are generally consistent with attention modulating the gain of sensory neurons in visual cortex. However, it is still unclear how attentional gain interacts with sensory inputs to selectively shape neuronal responses: While strong evidence suggests that attention imposes a multiplicative modulation of the sensory response characteristics of neurons, various studies report nonmultiplicative changes of neuronal responses (Moran and Desimone, 1985; Chelazzi et al., 1993; Luck et al., 1997; Recanzone and Wurtz, 2000; Treue and Maunsell, 1996, 1999; Reynolds et al., 1999; Connor et al., 1997; Womelsdorf et al., 2006; Williford and Maunsell, 2006).

Such non-multiplicative attentional effects are particular apparent for the influence of attention on the spatial tuning characteristics of neurons in extrastriate visual cortex. When attention is directed to different regions in space, neuronal receptive fields in these areas shift their center towards the attended location and narrow the width of spatial responsivity (Connor et al., 1997; Womelsdorf et al., 2006). This non-multiplicative modulation of the center and width of spatial tuning in single neurons stands in marked contrast to the multiplicative influence of attention on the tuning to features such as orientation or direction of motion of neurons in the same visual areas (McAdams and Maunsell, 1999; Martinez-Trujillo and Treue, 1999; 2004). For example, when attention is directed inside versus outside of the receptive fields of MT neurons, their bell shaped tuning functions for the direction of motion do not change their center and width, but are rather multiplicatively scaled by attention (Martinez-Trujillo and Treue, 1999).

Here, we attempt to reconcile these contrasting effects of attention by investigating the influence of selective attention on the spatial tuning functions of MT neurons. We find that attentional modulation of the center and the width of spatial tuning of MT neurons are correlated. We develop an attentional gain model that accounts for this correlation by assuming a bell-shaped spatial attentional spotlight imposing multiplicative spatial weighting on the sensory inputs of MT neurons. By using a 
Gaussian bell-shaped function we exploit the fact that the result of a multiplicative interaction of two Gaussians still is a Gaussian. The model also allows us to reverse-engineer the spatial distribution of the attentional influence on the neuronal population in area MT, predicting that a coarse resolution of spatial attention, i.e. a large spotlight of attention underlies the spatially selective modulation of receptive fields in visual cortex.

\section{Materials and Methods}

\section{Experimental set-up and visual stimulus}

We recorded from 78 neurons in area MT of the visual cortex of two macaque monkeys ( $n=57$ and $n=21$ cells in monkey $R$ and $D$, respectively) in a spatial attention task and obtained high-resolution receptive field maps under several conditions of spatial attention (see below). Details of the methods and procedures have been provided elsewhere (Treue and Maunsell, 1999; Womelsdorf et al., 2006). In short, recordings were done with tungsten electrodes (impedance 1.0 4.0 $\mathrm{M} \Omega$, Frederick Haer.) and cells isolated with a window discriminator (BAK Electronics or Plexon Inc.). Cells were localized in area MT by their physiological characteristics and the histological reconstruction of recording sites in monkey $R$. Access to MT was provided by a craniotomy and a recording chamber surgically implanted above the superior temporal sulcus of the left hemisphere. During the experiment, a custom computer program running on an Apple Macintosh PowerPC controlled stimulus presentation, and monitored and recorded eye positions and neuronal and behavioral responses. Eye positions were determined using a highresolution, video-based eye tracking system (ET49, Thomas Recording $\mathrm{GmbH}$ ) with a sampling frequency of $230 \mathrm{~Hz}$, and were digitized and stored at $200 \mathrm{~Hz}$. We used as stimuli moving random dot patterns (RDPs) of small bright dots (density: 10 dots per $\left.\mathrm{deg}^{2}\right)$ plotted within a stationary circular aperture on a dark $(0.7 \mathrm{~cd} /$ $\mathrm{m}^{2}$ ) computer monitor. Dots had an unlimited lifetime and were re-plotted at the opposite side of the aperture when they left the aperture during motion.

\section{Experimental Paradigm}

We used a spatial attention task illustrated in figure 1A. A trial started once the animal foveated a small square presented on a computer screen. Then the cue, a stationary random dot pattern (with $19 \mathrm{~cd} / \mathrm{m} 2$ bright dots) was presented for 440 ms and after a brief blank delay three moving RDPs were shown. Two of them (S1 and S2) were positioned within similarly activating regions within the receptive field of the isolated neuron and always at equal eccentricity, while the third RDP (S3) was presented outside the receptive field in the opposite hemifield. The task was to detect a small, transient $(80 \mathrm{~ms})$ change of the direction of motion of the stimulus at the cued location. Changes of this target stimulus and the distractors occurred at random times between 90 and $4090 \mathrm{~ms}$ after onset of S1, S2, and S3. 
Change times were drawn from a flat uniform distribution. During the sustained state of selective spatial attention we obtained high-resolution receptive field maps by recording the responses to a probe stimulus of higher-contrast $\left(47 \mathrm{~cd} / \mathrm{m}^{2}\right)$ successively presented for $190 \mathrm{~ms}$ at the intersections of a dense virtual grid adjusted to cover the receptive field and its immediate surround. Potential probe positions that would have caused the probe to overlap S1 or S2 were not used (see fig. 1A). The sequence of probe positions was randomized for each trial. S1, S2, and S3 moved in the anti-preferred direction of the neuron and were of intermediate contrast $(19 \mathrm{~cd} / \mathrm{m} 2)$, while the probe stimulus moved in the preferred direction and had higher contrast (42 cd / m2). Temporal intervals in which no probe was shown were included in the succession of probe presentations to determine the baseline response (evoked by the two anti-preferred stimuli in the RF). Our experimental design allowed us to map the receptive field during sustained states of selective attention to either of the stimuli inside the receptive field (attend-inside conditions) or to the stimulus outside the receptive field (attendoutside condition). For the current study we performed for each neuron the two experimental comparisons of switching attention from outside the RF (S3) to either the one (S1) or the other (S2), stimulus inside the receptive field.

\section{Data Analysis}

We quantified the spatial structure of neuronal receptive fields by using the average response to the probe beyond the baseline activity at each grid intersection in an interval from $60-200 \mathrm{~ms}$ after probe-onset. The twodimensional grid of neuronal firing rates in response to the probe was analyzed non-parametrically (by spline interpolation of the response surface), as well as parametrically by fitting the response profile with a two dimensional Gaussian model of the form:

$$
\begin{aligned}
f(x, y)=B+ & A \exp \left(-\frac{\left[\left(x-x_{0}\right) \cos \theta+\left(y-y_{0}\right) \sin \theta\right]^{2}}{2 \sigma_{x}^{2}}\right. \\
& \left.-\frac{\left[-\left(x-x_{0}\right) \sin \theta+\left(y-y_{0}\right) \cos \theta\right]^{2}}{2 \sigma_{y}^{2}}\right)
\end{aligned}
$$

with $f(x, y)$ reflecting the response to the probe at spatial position $(x, y),\left(x_{0}, y_{0}\right)$ being the receptive field center, $\theta$ the orientation of its main elliptical axis, and $\sigma_{x}$ and $\sigma_{y}$ the standard deviations of the two axes. This model has been successfully applied in previous studies to describe receptive fields of MT neurons (Raiguel et al., 1995; Britten and Heuer, 1999). The fitting was performed by minimizing the Chi square value between model and data. For the spline-interpolated surface we computed the receptive field center by determining as the center of mass of the 
A

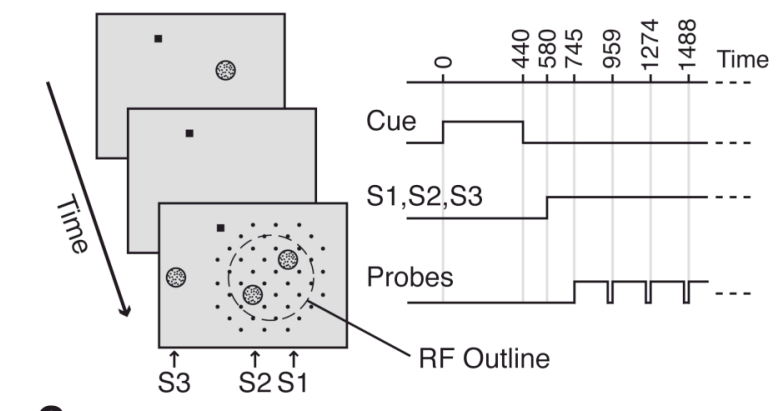

C

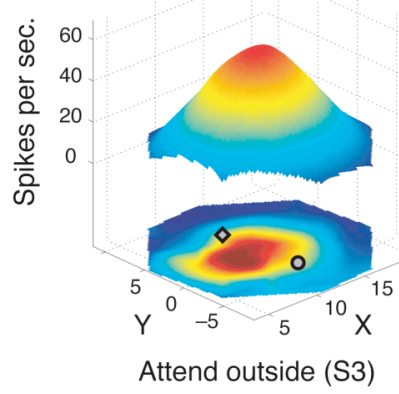

Attend inside (S1)
B

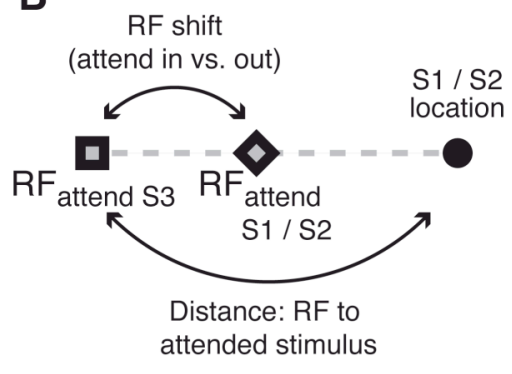

$\diamond$ Stimulus S1 O Stimulus S2

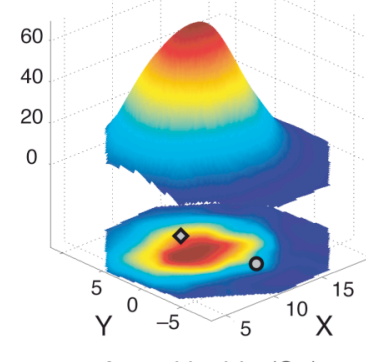

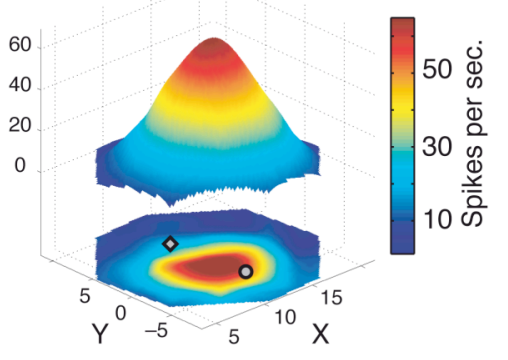

Attend inside (S2)

Figure 1: Experimental protocol and illustration of receptive field maps. A: Experimental layout showing the succession of cue, target stimulus (in this example: S1 inside the receptive field outline (dashed circle)), distractor stimuli (inside receptive field: S2, and outside receptive field: S3) and the possible locations of the probe stimulus (black dots). B: Illustration of the calculation of receptive field shift (distance of the receptive fields in the attend outside and attend inside conditions) proportional to the distance of the receptive field (attend outside) to the attended stimulus position. C, Example receptive fields of a neuron while attention was directed outside the receptive field to S3 (left panel), inside the receptive field to stimulus S1 (middle panel), and to stimulus S2 (right panel). The bottom maps show spline-interpolated neuronal response profiles and the floating 3-D maps are the Gaussian fits used to describe the receptive field parameters. The example illustrates that responses to probes close to (far from) an attended stimulus in the receptive field were enhanced (reduced) compared to when attention was directed to S3.

area above half-maximum response, and the size of the receptive field as the square root of that area.

We evaluated the goodness of fit to the response profiles of the neurons in two steps. First we removed fits that did not provide a better description of the response profile than the variability around the average response of the neuron by comparing the residuals to the global mean using the Kruskal-Wallis test. For neurons not exceeding the $95 \%$ significance level the fitted receptive field surface represented the data not better than a surface based on the variability underlying the average activity level. In a second step we removed receptive field fits that strongly deviated (see below) in at least one of the free parameters with regard to those of all other receptive field fits (baseline, amplitude, center position and sigma, i.e. the average of the tuning width along the major and minor axis of the receptive field). We used an incremental procedure to eliminate those outliers by excluding fits from the final analysis where any of the parameters exceeded 4 standard deviations of the overall variation in that parameter. This two-step 
procedure eliminated 24 out of 119 pairs (20.2\%) of the attend-in and attend-out condition (see Results).

We evaluated shifts of the receptive field center in the attend-in versus attend-out condition by treating the receptive field location in the attend-out condition as the reference position $\left(R F_{\text {out }}\right)$. We calculated the distance of this reference position (i) to the receptive field center when attention was directed inside the receptive field ( $R F^{\text {attended }}$ ), and (ii) to the attended stimulus position inside the receptive field. We defined the receptive field shift as the distance between $R F_{\text {out }}$ and $R F^{\text {attended }}$ proportional to the distance between $R F_{\text {out }}$ and the attended stimulus position inside the receptive field (cf. fig. 1B), defining positive (negative) shift values to be toward (away from) the attended stimulus. To quantify changes in the amplitude of the Gaussian (i.e. the maximal firing rate of the spline-interpolated surfaces) and size of the receptive fields, we computed the ratio of the values of the attend-in and attend-out conditions. All analysis and model calculations were done with Matlab (MathWorks Inc., MA).

\section{Model}

We propose a spatial multiplicative model using modulatory attentional signals on feed-forward sensory inputs. For simplicity of presentation, we restrict the model to one-dimensional space. Sensory feed-forward spatial inputs are modeled with a Gaussian connectivity profile (receptive field center $x_{R}$, amplitude $A_{R}$ and size $\sigma_{R}$ ). Attention is acting multiplicatively on these connections and the model assumes a bell shaped function of spatial attention. Here, we restrict the model to a Gaussian bell shape of the attentional spotlight with center $x_{A t t}$, amplitude $A_{A t t}$ and size $\sigma_{A t t}$. The spatial response profile of a neuron (the "output RF") is the product of two functions, the receptive field profile of its input ("input RF") multiplied by attention with the connectivity profile

$$
1+A_{A t t} \exp \left(-\frac{\left(x-x_{A t t}\right)^{2}}{2\left(\sigma_{A t t}\right)^{2}}\right)
$$

When attention is weak $\left(A_{A t t}<1\right)$, input and output receptive fields are very similar. When attention is strong $\left(A_{A t t}>1\right)$, its influence has a Gaussian profile with a negligible baseline. Because the receptive field in the attended condition ( $R F^{\text {attended }}$ ) is the result of a multiplication of the Gaussian input RF and the attentional Gaussian connectivity profile, we can calculate the parameters

$$
R F^{\text {attended }}=A_{R} A_{A t t} A^{\text {attended }} \exp \left(-\frac{\left(x-x_{R}^{\text {attended }}\right)^{2}}{2\left(\sigma_{R}^{\text {attended }}\right)^{2}}\right)
$$




$$
\begin{gathered}
x_{R}^{\text {attended }}=\frac{\frac{x_{R}}{\left(\sigma_{R}\right)^{2}}+\frac{x_{A t t}}{\left(\sigma_{A t t}\right)^{2}}}{\frac{1}{\left(\sigma_{R}\right)^{2}}+\frac{1}{\left(\sigma_{A t t}\right)^{2}}} \\
\frac{1}{\left(\sigma_{R}^{\text {attended }}\right)^{2}}=\frac{1}{\left(\sigma_{R}\right)^{2}}+\frac{1}{\left(\sigma_{A t t}\right)^{2}} \\
A^{\text {attended }}=\exp \left(-\frac{\left(\sigma_{R}^{\text {attended }}\right)^{2}\left(x_{R}-x_{A t t}\right)}{2}\right)
\end{gathered}
$$

with $x$ corresponding to the spatial position of the sensory inputs, and $A_{R}$ and $A_{A t t}$ corresponding to the amplitudes of the receptive field and attention respectively (see Supplemental Material for details). The proposed model makes no assumptions or predictions about the height of the various curves, as cortical normalization processes tend to keep the volume under the receptive field response surface constant (Croner and Kaplan, 1995), i.e. create multiplicative changes to the receptive field amplitude without changing the location or width parameters. Such a normalization process is supported by our data (for details see Supplementary Material; Supplementary fig. 1). We therefore did not incorporate the normalization process in our model. Equation 4 represents the new receptive field center, and equation 5 the new receptive field size. Note that the shift and shrinkage of the receptive fields with attention is calculated as $\left(x_{R}^{\text {attended }}-x_{R}\right) /\left(x_{A t t}-x_{R}\right)$ and $\sigma_{R}^{\text {attended }} / \sigma_{R}$, respectively. From these terms and equations $4-5$, the model predicts a fixed relation of changes in receptive field center ('shift') and changes in receptive field size ('shrinkage') according to:

$$
\text { shrinkage }=\sqrt{1-\text { shift }}
$$

This equation allows disregarding the width of the attentional influence and the receptive field size and position by representing the final result as the relation between the observed receptive field parameter changes due to spatial attention. In addition, knowing the location of attention and the size of the output RF with attention outside the receptive field, we can calculate the halfwidth of the spatial spread of the attentional influence $\sigma_{A t t}$ according to:

$$
\sigma_{A t t}=\sigma_{R} \sqrt{\frac{1}{\text { shift }}-1},
$$

as long as the model's prediction that RFs only shift toward the attentional center is correct. 


\section{Bootstrap confidence interval and Monte-Carlo simulations}

Equation 7 describes the theoretical relation between receptive field shift and shrinkage. The first statistical test we performed for comparing a given experimental receptive field shift-shrinkage value to the theoretical value was through bootstrapping the raw receptive field profiles in the attend-in and attendout conditions and assessing the confidence interval of both shift and shrinkage (e.g. Efron and Tibshirani, 1993). To this end we bootstrapped the individual receptive fields before fitting, and then restricted further extraction of the size and center values to those receptive fields of the bootstrap sample with an R-square value above the median. These receptive fields were then used to calculate a random distribution $(n=1000)$ of receptive field shrinkage and shift for a given pair of receptive fields to obtain the $95 \%$ confidence range for that pair. This analysis tests if a given single neuron data follow a theoretical behavior given the chosen experimental sampling of the 2-D visual space and the observed variability.

In a second statistical approach we computed the expected variability on the theoretical model predictions by performing a Monte-Carlo simulation of repeated experimental measurements of a theoretical Gaussian receptive field profile modulated by a Gaussian-shaped attentional spotlight. We simulated the experimental mapping of the receptive fields in the attend-in and attend-out conditions by using the mean parameters across all experiments together with their variability. This statistical approach tests how much variability should be expected from a given theoretical model given the known experimental conditions. Therefore, it allows estimating the expected theoretical distribution of the data around the mean model predictions, and it allows testing if the data follow the theoretical model. The experimental set-up and measurement procedure were simulated as follows: A virtual grid of 7 by 7 degrees with a step size between adjacent points of 1 degree was chosen; receptive field centers and sizes in the attend-out condition were generated according to the experimental distribution ( $\log _{10}$ of sigma_out / "Grid step size" $=0.20 \pm 0.17$; mean $\pm S D$ ); position of the initial receptive field center with respect to the grid center was randomized (SD of 0.3 for a step size of 1.0); attention was centered with respect to the receptive field center (at one standard deviation from the receptive field center plus the variability around this position from neuron to neuron, SD of 0.25 ); firing rate variability was included $\left(\log _{10}\right.$ of the SD over the mean rate: $\left.-0.10 \pm 0.24\right)$; a background firing rate was added to the neuronal receptive field profile ( 1 / 10 of the maximal firing rate). For each of the 10,000 simulations of the experiment at a given shift value (from $1 \%$ up to $90 \%$ in steps of $1 \%$ ), the attentional and initial receptive field parameters (center and size) were randomly drawn from the corresponding distributions and firing rate variability included. The resulting receptive field profiles in the attend-out and attend-in conditions were fitted like the experimental data. In each case we assessed the $99 \%$ expected variability due to these experimental conditions. 


\section{Eye position measurement}

Eye positions were measured with a high-resolution, video-based eye tracking system (ET49, Thomas Recording $\mathrm{GmbH}$ ) with a sampling frequency of $230 \mathrm{~Hz}$, and were digitized and stored at $200 \mathrm{~Hz}$. In order to test for a potential systematic contribution of changes in eye positions between attention conditions to the receptive field changes we calculated the average eye position difference between the attend-inside conditions and the attend-outside condition along the axis of the respective stimuli (i.e. along the axis from S1-to-S3 or from S2-to-S3). We found a very small difference of eye positions of only $0.009^{\circ}\left( \pm 0.004^{\circ} \mathrm{SE}\right)$ in the direction of the attended stimulus, which is far smaller than the difference in receptive field position between conditions (see below). These findings suggest that changes in eye position contributed marginally at best to changes in spatial neuronal sensitivitiy (cf. also Womelsdorf et al., 2006).

\section{Results}

\section{Determining the dataset of neuronal receptive field pairs}

We mapped the receptive fields of 78 single neurons in area MT of two monkeys while they directed attention to one of two stimuli inside (attend-in condition) or to a stimulus outside (attend-out condition) the receptive field. For 64 neurons the receptive field of the attend-out and the receptive field of at least one of the two attend-in conditions could be fit by a two-dimensional Gaussian (for an example: fig. 1C) (see Materials and Methods for details). To capture in detail the quality of the fits in the remaining 97 pairs of receptive fields (65 and 32 pairs from monkey $R$ and $D$, respectively) in the attend-in and attend-out condition we binned the $R$ square values of the individual receptive field fits (fig. $2 A$ ). The distribution of $R$ square values (median: 0.788) demonstrates that the majority of the response profiles could be well modeled by a Gaussian profile despite the continuous presence of two anti-preferred stimuli within the receptive field, limiting the sampling to regions outside of these stimuli. In order to exclude poor fits caused by non-optimal spatial sampling from further analysis we opted for a conservative inclusion criterion of the fits by focusing on those receptive field pairs, where each fit exceeded the median R-square value of 0.788 ( $n=27$ pairs). In the following these pairs are termed the selected receptive field pairs. For comparison we will also present results of all those remaining fitted pairs $(n=70)$, where at least one receptive field fit showed a $\mathrm{R}$-square value below the median. Examples of fits with $\mathrm{R}$-square values above the median are presented in figure $2 \mathrm{~B}-\mathrm{C}$ and example fits with R-square value below the median are shown in figure 2D-E. Moreover, to ease comparison with non-parametric analysis from previous studies we additionally analyzed changes in receptive field parameters from the splineinterpolated receptive field profiles (bottom panels in figure $2 B-E$ ). 

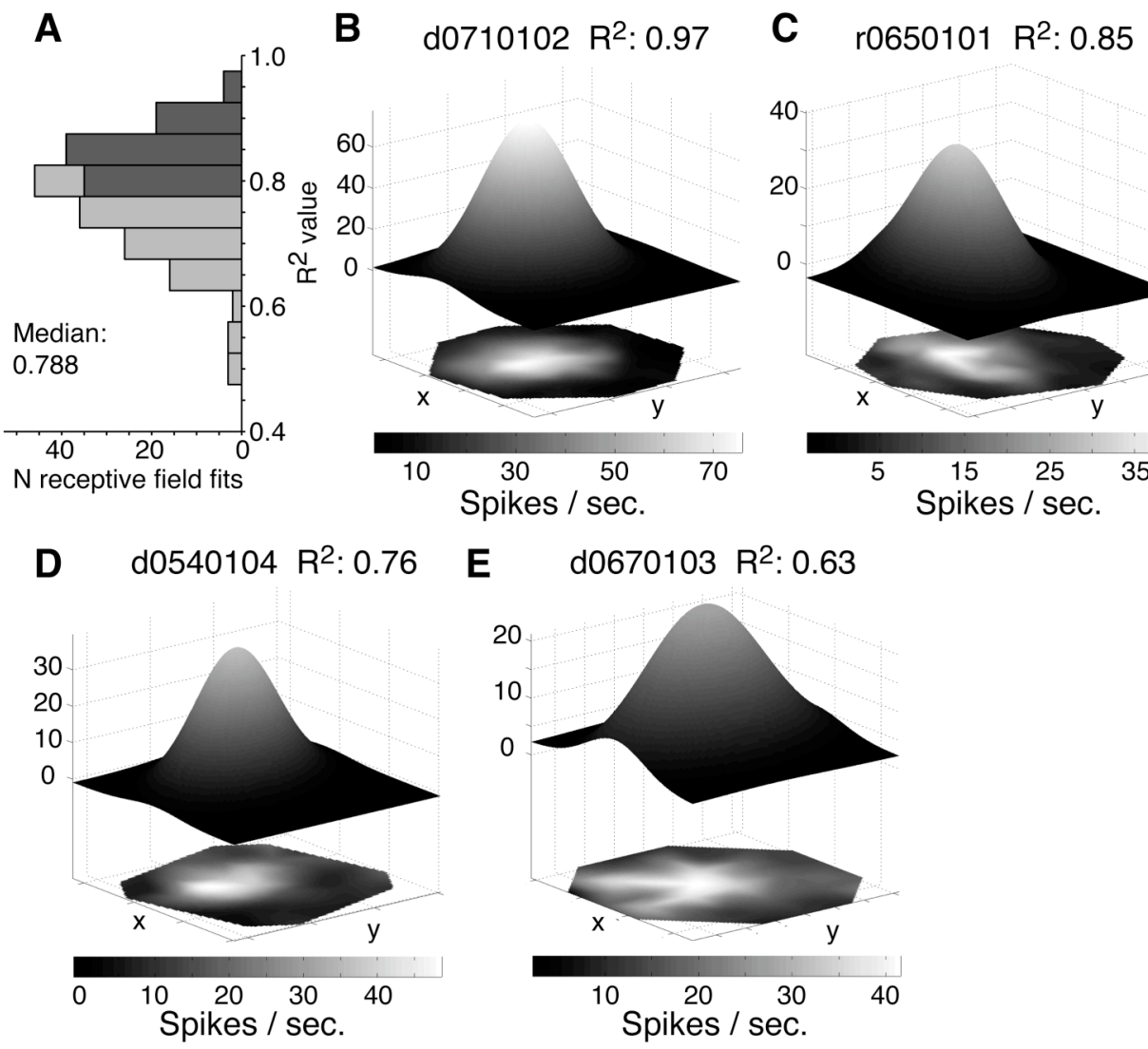

E $\quad$ d0670103 $R^{2}: 0.63$
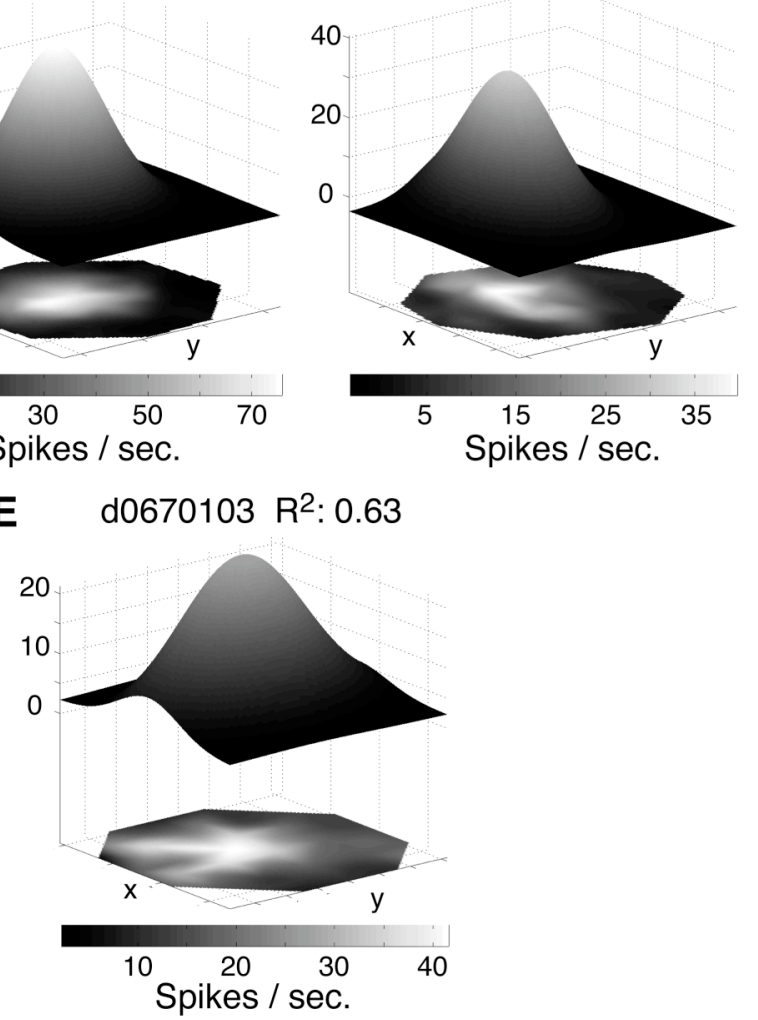

Figure 2: Fitting MT receptive fields with a Gaussian profile. A: Distribution of R-square values of the Gaussian fits to individual receptive fields in the different attentional conditions. Bar coloring indicates fits with an above-median (dark grey) and below-median (light grey) R-square value. B-E: Examples of spline-interpolated (bottom surfaces in two dimensions) and fitted (upper threedimensional surfaces) receptive fields profiles with an above-median R-square value (B-C), and a below-median R-square value (D-E).

\section{Attentional effects on neuronal receptive fields}

Across the population of receptive field pairs (monkey R: 65 pairs, monkey D: 32 pairs) we observed three effects: a shrinkage of the receptive fields and a moderate increase in the receptive field amplitude when attention was switched from outside to inside the receptive field. When attention was switched between the two locations inside the receptive field we found a substantial shift in the location of receptive field center. Figure 3 shows the distribution of the three effects. Since the two monkeys each showed the same effects for the three modulations we pooled the receptive field pairs for further analysis and illustration of results and give the individual monkeys' values below. Figure $3 \mathrm{~A}$ shows the distribution of the relative change in maximal response (receptive field amplitude) with attention inside versus outside the receptive field. For the 27 selected receptive field pairs (dark grey bars in fig. $3 \mathrm{~A}$ ) the average neurons' peak response was $4.7 \%( \pm 4 \% \mathrm{SE}, \mathrm{n}=27)$ higher with attention inside vs. outside the receptive field but this effect failed to reach significance $(p=0.21$, one sample ttest). Similarly, the modulation of the peak response was not significant when all pairs of receptive field fits were considered (figure $3 \mathrm{~A}$, light plus dark grey bars, 


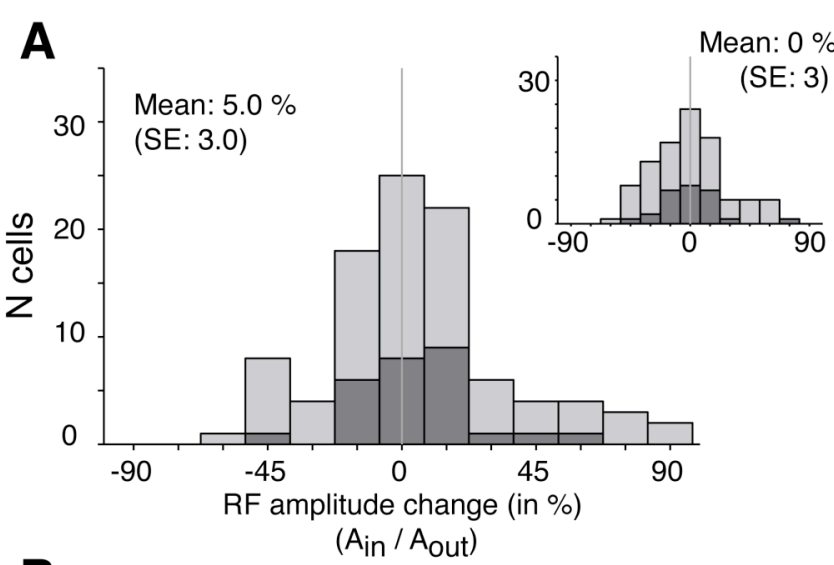

B
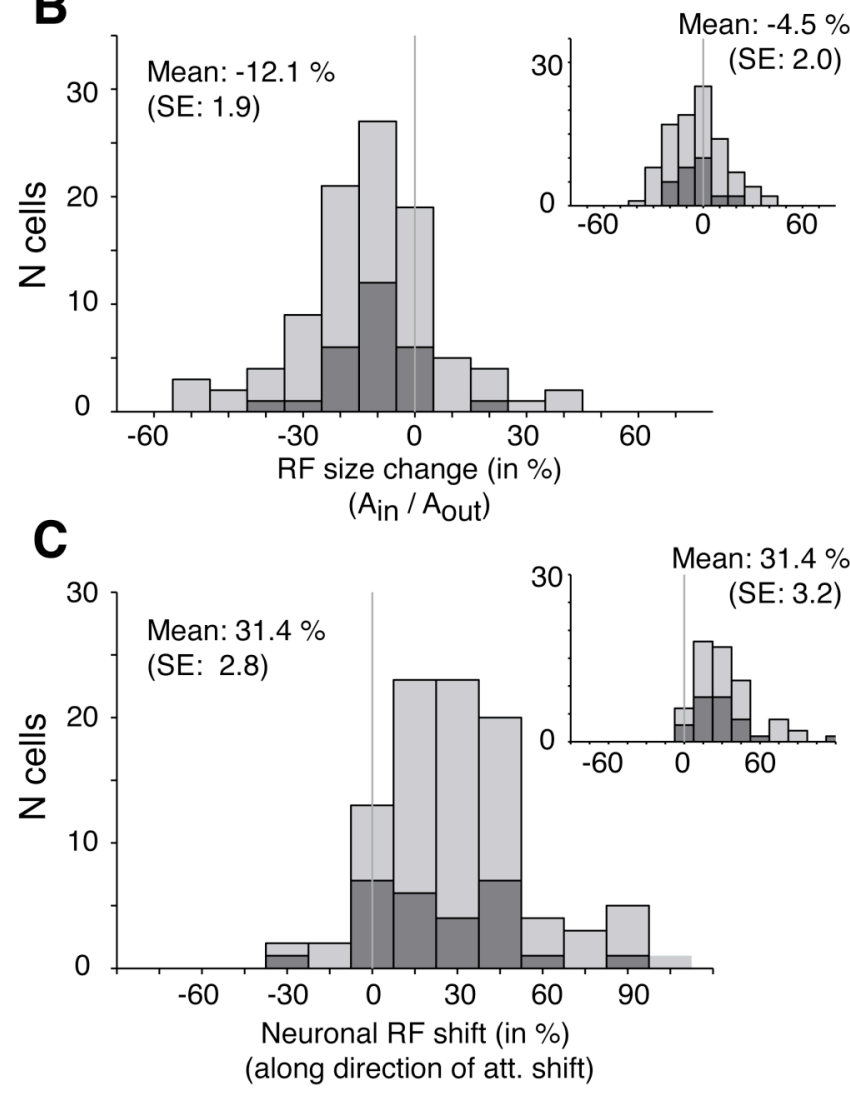

Figure 3: Neuronal receptive field modulation with attention inside versus outside the receptive field (RF) for $97 \mathrm{RF}$ pairs (in each panel mean and SE are computed across all $97 \mathrm{RF}$ pairs). The dark grey colored bars indicate "selected" RF pairs, i.e. those pairs where both RFs were fitted with above-median R-square values $(n=27) . A$ : Distribution of changes in RF amplitude between conditions (Gaussian-fitted RFs). The inset plot shows changes in amplitude (maximum response to the probe) extracted from the splineinterpolated RFs. B: Histogram of receptive field size changes with attention inside versus outside the receptive field. Values below zero indicate receptive field shrinkage. The inset shows the size changes when RF size is measured as the square root of the area above the half maximum response in the interpolated profile. C: Distribution of receptive field shifts parallel to the axis connecting the receptive field center when attention was directed to S3 and the target location in the paired condition when attention was directed into the receptive field (long arrow in figure 1B). Positive values signify shifts toward the attended stimulus inside the receptive field. The inset shows the RF shift distribution obtained from the nonparametric maps (with RF centers measured as the center of mass of the area at half-maximum response).

mean: $5 \%, p>0.14, n=97$; monkey R: $4.1 \% \pm 4.3 \%$, monkey D: $5.1 \% \pm 2.2 \%$, and when the modulation was based on the peak response obtained from the nonparametric analysis (mean: $0 \%(1 \%)$ for the selected (all) pairs; see inset in figure $3 \mathrm{~A})$. The wide range of modulations of the receptive field amplitude might be partially due to the influence of a normalization process (see methods section and suppl. material).

Determining changes in receptive field size we observed a highly significant shrinkage of $-11.2 \%( \pm 2.3 \%, p<0.001$, one sample t-test, $n=27$, fig. $3 B$ ) with attention inside versus outside the receptive field for the selected pairs (for the whole sample: $-12.1 \% \pm 1.9 \%, n=97$; monkey $\mathrm{R}:-14.7 \% \pm 2.5 \%$, monkey $\mathrm{D}$ : $7.8 \% \pm 2.6 \%)$ ). This shrinkage is significantly larger than obtained for the same sample with the nonparametric analysis (mean of $-4.5 \%$ inset in fig. $3 B, n=97$ ) 
and compared to the $-4.3 \%$ population average we reported previously based on a nonparametric analysis of all receptive field pairs (Womelsdorf et al., 2006). Even the shrinkage based on the non-parametric analysis was significant $(p<0.05$, one sample t-test). Note that the latter analysis used the square root of the area above the half maximum response to the probes compared to the use of the sigma parameter for the Gaussian fitted response profiles. Because of the shrinkage of the receptive fields the increase in receptive field amplitude does not reflect an overall gain change as the neurons' responsiveness dropped for positions away from the center of the receptive field.

Figure $3 \mathrm{C}$ shows the effect of attention on the receptive field positions. On average receptive field centers for the selected pairs were shifted $25.3 \%( \pm 4.6 \%$, $p<0.001$, one sample t-test, $n=27$, dark grey bars in fig. $3 C$ ) toward the stimulus inside the receptive field when it was attended compared to when attention was directed to the stimulus in the opposite hemifield. The average shift value for the whole sample (light and dark grey bars in figure $3 \mathrm{C})$ of $31.4 \%( \pm 2.8 \%, p<0.001$, one sample t-test, $\mathrm{n}=97$; monkey $\mathrm{R}: 38 \% \pm 3.6 \%$, monkey $\mathrm{D}$ : $18 \% \pm 2.8 \%$ ) was non-significantly larger. The $25.3 \%$ and $31.4 \%$ proportional shifts of receptive fields correspond to an average receptive field shift of 1.43 and 2.56 degrees of visual angle respectively. Similar average receptive field shifts were obtained for the interpolated maps, i.e. when the receptive field center was measured as the centroid of the area above the half-maximum response (fig. $3 \mathrm{C}$, inset): On average receptive fields shifted $27.9 \%(31.4 \%)$ for the selected (and the whole) dataset (both: $p<0.001$, one sample t-tests).

Intriguingly, the shift of receptive fields toward the attended focus and the narrowing of the spatial tuning show a highly significant correlation $(r=-0.55, p<$ 0.01 , red data points in fig. $4 \mathrm{C}$ ), i.e. neurons with larger receptive field shifts toward the attended stimulus showed stronger receptive field shrinkage. The correlation of receptive field shift and shrinkage also hold for the whole sample of fitted receptive field pairs $(r=-0.38, p<0.001$, red plus grey data points in fig. $4 \mathrm{C}$ ), as well as for the RF shift and shrinkage correlation calculated on the basis of non-parametric receptive field profiles $(r=-0.56, p<0.01$, red data points in fig. 4D).

\section{Spatially selective gain modulation}

The reported modulation of receptive field position and size by spatial attention reflects a non-multiplicative change of the spatial tuning characteristics of individual MT neurons. To test whether the observed findings nevertheless could be reconciled with a multiplicative attentional modulation we created a general gain model assuming that attention multiplicatively modulates the spatial weighting of inputs to MT from a mosaic of cells with smaller receptive fields. By assuming that spatial attention is distributed with a Gaussian profile centered on the 
A

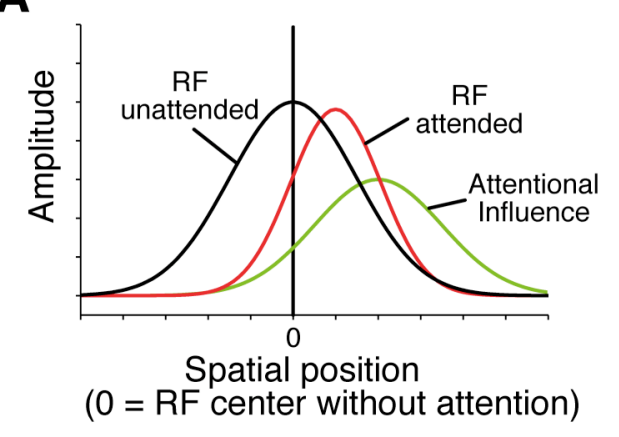

C

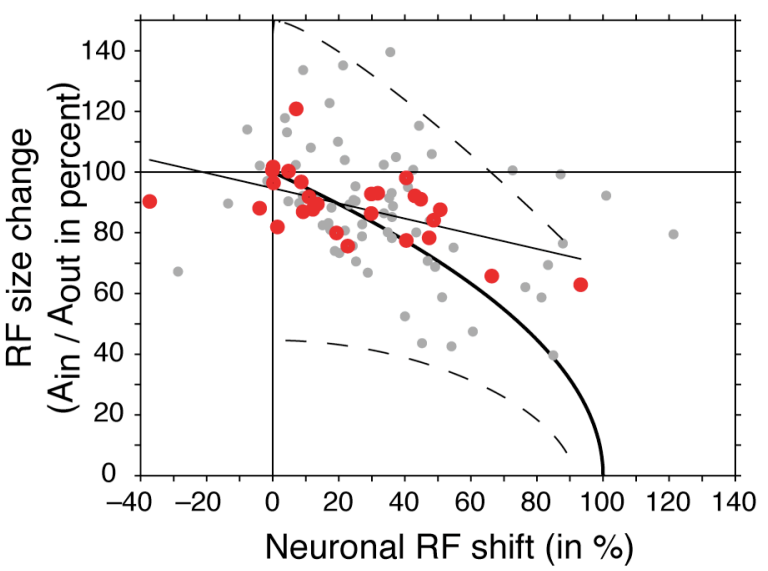

B

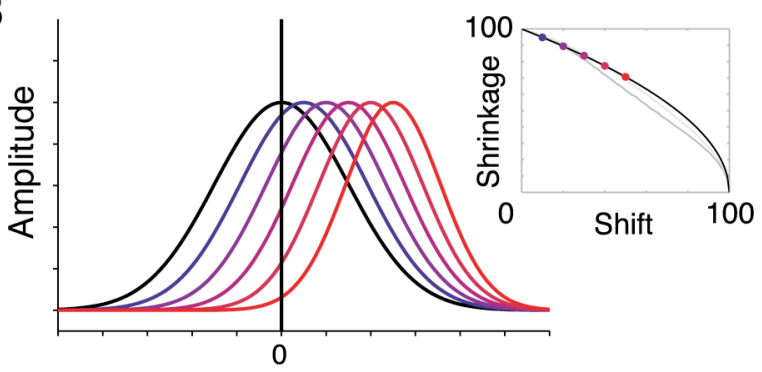

Spatial position

( 0 = RF center without attention)

D

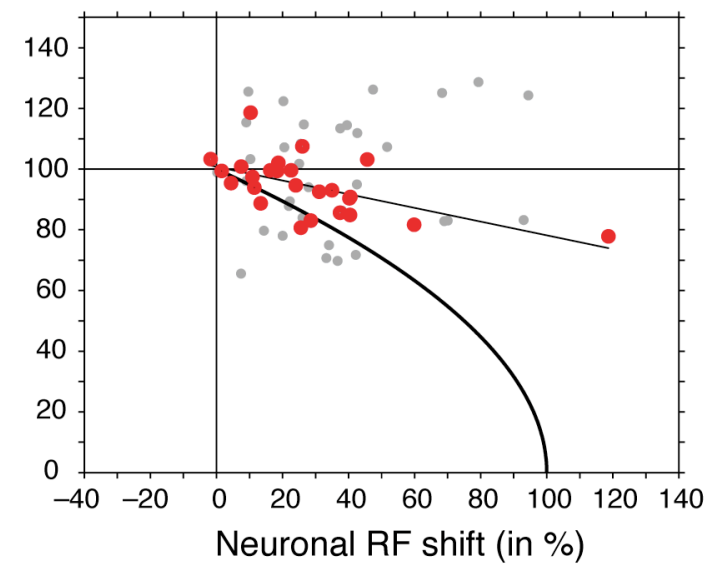

Figure 4: Illustration of model assumptions and the model's fit to the neuronal data. A: The model predicts that the spatial response profile of a neuronal receptive field (here as 1-D Gaussians) with attention outside the receptive field (black line: RF unattended) interacts multiplicatively with a Gaussian shaped attentional influence (green line). Multiplying these two Gaussians result in a narrower receptive field, which is shifted toward the center of attention (red line: RF attended). B: Illustration of the predicted relation of the extent of neuronal receptive field shift and receptive field shrinkage (see eq.7). The stronger the shift of neuronal receptive fields (blue to red (left to right) Gaussian receptive field profiles) the stronger the receptive field shrinkage following eq.7 and shown in the inset (blue to red dots). The black line in the inset illustrates the predicted shift and shrinkage relation according to eq. 7, assuming a Gaussian shaped receptive field and a Gaussian shaped attentional spotlight. Grey lines show the prediction under the assumption of a Cosine shaped attentional spotlight together with receptive fields shaped as Gaussian (dark grey) or Cosine functions (light grey). C: Scatter plot of the observed receptive field shrinkage (y-axis; $100 \%$ represents no size change) and receptive field shift ( $x$-axis) for 27 pairs of receptive fields in the attend-in versus attend-out conditions which could be fit with an R-square value above the median (red colored dots), and for the remaining 70 pairs (grey dots). The black line shows the linear regression, while the solid black curve indicates the relation of receptive field shift and shrinkage predicted by the model. Dashed lines shows the $99 \%$ confidence range expected for data points across all cells (based on the Monte-Carlo simulation, see Methods). D: Same format as in $\mathrm{C}$, but plotting the results based on the comparison of the non-parametric estimates of the receptive field center (center of mass) and size (square root area above half-maximum response).

attended stimulus' location the model creates MT receptive fields by the multiplicative interaction of the Gaussian-shaped input receptive field with the Gaussian-shaped input weights. Because multiplying two Gaussians results in a Gaussian, the MT receptive field profile with attention remains Gaussian shaped, with its width and center position being a function of the distance between the original two Gaussians and their respective widths (for an illustration see fig. 4A). 
The model predicts that receptive field shift and shrinkage are correlated such that receptive fields shrink with attention inside the receptive field proportional to $\sqrt{1-R F s h i f t}$ (fig. 4B, see Materials and Methods), i.e. a strong shift is accompanied by a strong shrinkage of receptive fields. Note that the predicted correlation of receptive field shift and shrinkage does not rely on the assumption of a Gaussian function for receptive fields and the attentional spotlight. Rather, a positive correlation of shift and shrinkage occurs for the interaction of other bell shaped functions (e.g. Cosine shapes) as well. Figure 4B (inset, grey curves) illustrates that this basic relationship is also predicted when assuming either a cosine shaped attentional spotlight, or a cosine shape for receptive fields and attentional spotlight. Different bell shaped functions vary primarily in the predicted slope of the relation of RF shift and shrinkage as one approaches RF shifts larger than about $40 \%$. We therefore restrict our analysis to the assumption of Gaussian receptive field shape and a Gaussian attentional spotlight, which has the advantage to allow for straightforward model equations (see Methods).

Figure 4C shows the observed correlation of receptive field shift and shrinkage along with the predicted correlation (solid black curve). For the selected receptive field pairs $70.3 \%$ (19 of 27 , red dots in fig. 4 C) of the data points had a $95 \%$ confidence range overlapping with the model prediction. Considering the remaining 70 pairs (grey dots in fig. 4C), the bootstrap analysis showed that $75.7 \%$ (53 of 70 ) of them significantly overlapped with the model prediction.

The bootstrap analysis provided a means to test whether individual data points can be accounted for by the model. In a second step we viewed the issue from the perspective of the model and asked how likely it would have been to observe individual data points that correspond to the model prediction if we would have repeated the experiment with more neurons and similar experimental settings by means of a Monte Carlo simulation (see Methods for details). The dashed lines in figure 4C shows the resulting upper and lower statistical bounds (99\% confidence level) of the variability of the shift-shrinkage relation that is caused by the variability in our experimental design applied to a simulated theoretical experiment. These bounds show that 24 out of the 27 selected points $(88.9 \%)$ could be accounted for by the model (red dots in fig. 4C). Considering the whole sample there were still $86.6 \%$ (84/97) of the observed shift-shrink relations that are statistically expected with $99 \%$ confidence. 
A

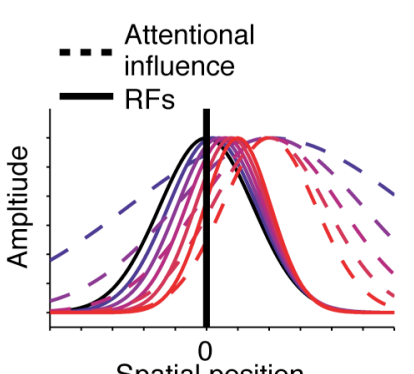

( 0 = RF center without attention)

D

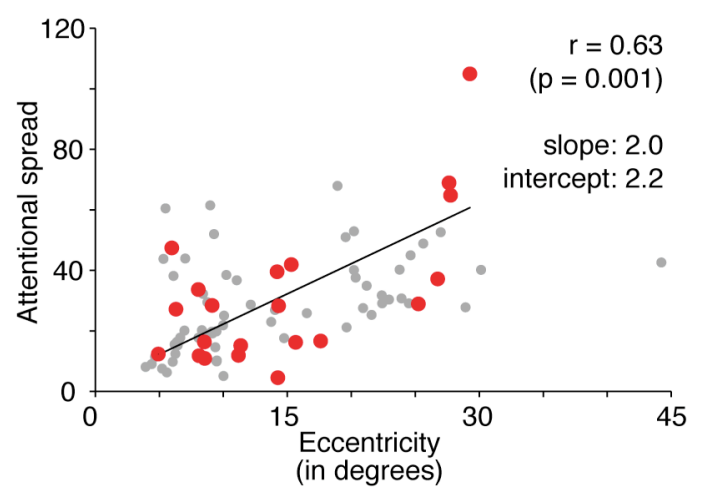

C
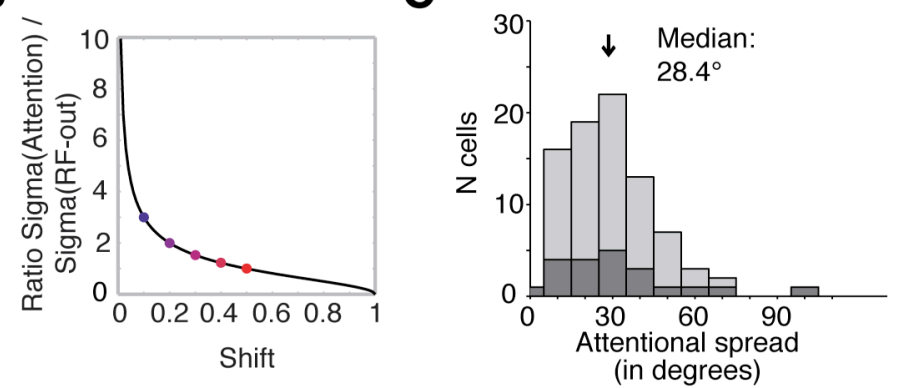

E

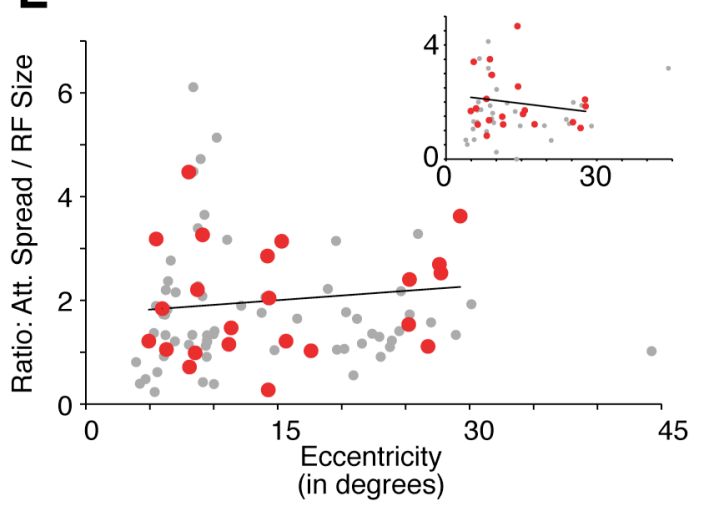

Figure 5: Model prediction and estimation of attentional spread underlying the observed shift and shrinkage of neuronal receptive fields. A: Given a fixed spatial center of attention, the model estimates a narrower attentional spread (blue to red dashed Gaussians) the stronger the receptive field is shifted towards the attentional focus (blue to red solid Gaussians). B: Relationship between the expected RF shrinkage (when attention is shifted into the RF) as a function of the observed RF shift (equation 8, see Methods). C: Distribution of the attentional spread (full width) estimated based on the observed RF shifts. Dark / light grey bars represent the data from the selected / all RF pairs as in fig. 2-4. D: Scatter plot showing that the estimated attentional spread (y-axis) grows with an increasing eccentricity of the attentional focus ( $x$-axis). The best fitting linear regression has the form width of attentional spread $=2.0 *$ eccentricity +2.2 . Red / grey dots represent the data from selected / all RF pairs as in fig. 2-4. E: Same format as in D, but showing the lack of correlation between the ratio of attentional spreads to RF size with eccentricity of the neuron's RF.

\section{Prediction of attentional spread}

In addition to the ability to account for the observed relationship between receptive field shift and shrinkage, the model allows us to estimate the spatial spread of the attentional influence given the observed modulation of neuronal spatial tuning (cf. equation 8 in Methods and Material). Figure 5A illustrates the predicted attentional spread underlying varying degrees of receptive field shifts and shrinkage, showing that narrower attentional width induces stronger modulation of receptive fields, that is, stronger shrinkage and shifts toward the attentional focus. The general relationship is presented in figure $5 \mathrm{~B}$, which plots the ratio of estimated attentional spread and receptive field size as a function of the observed shift. Figure 5B demonstrates that for receptive field shifts smaller than about 0.3 the attentional widths are expected to be far broader than the receptive field size. Note that the described relation is valid only for receptive field shifts toward the attended position and not beyond it, i.e. only for shifts between 0 and $100 \%$. Using this relationship we estimated the attentional spread underlying each of the observed receptive field shifts (fig. 5C, D). We observed a median full width of the attentional 
spread of $27.4^{\circ}$ (mean: $28.4^{\circ} \pm 3.8^{\circ}$ ) for our sample of selected receptive fields (median / mean full-width RF size: $16.4^{\circ} / 18.6^{\circ}$ ) indicating that the attentional spread is on average about $60 \%$ larger than the size of MT receptive fields.

In addition, there was a significant positive correlation of the width of the attentional influence with the eccentricity of the RFs (red data points in fig. 5D, $r=$ $0.64, p<0.001$ ): Attentional spread grows from about $2.2^{\circ}$ near the fovea to about $60^{\circ}$ at an eccentricity of $30^{\circ}$ (regression intercept: 2.2 , slope: $2.0^{\circ}$ ), indicating that the spotlight of attention increases linearly with eccentricity of attention. Note that

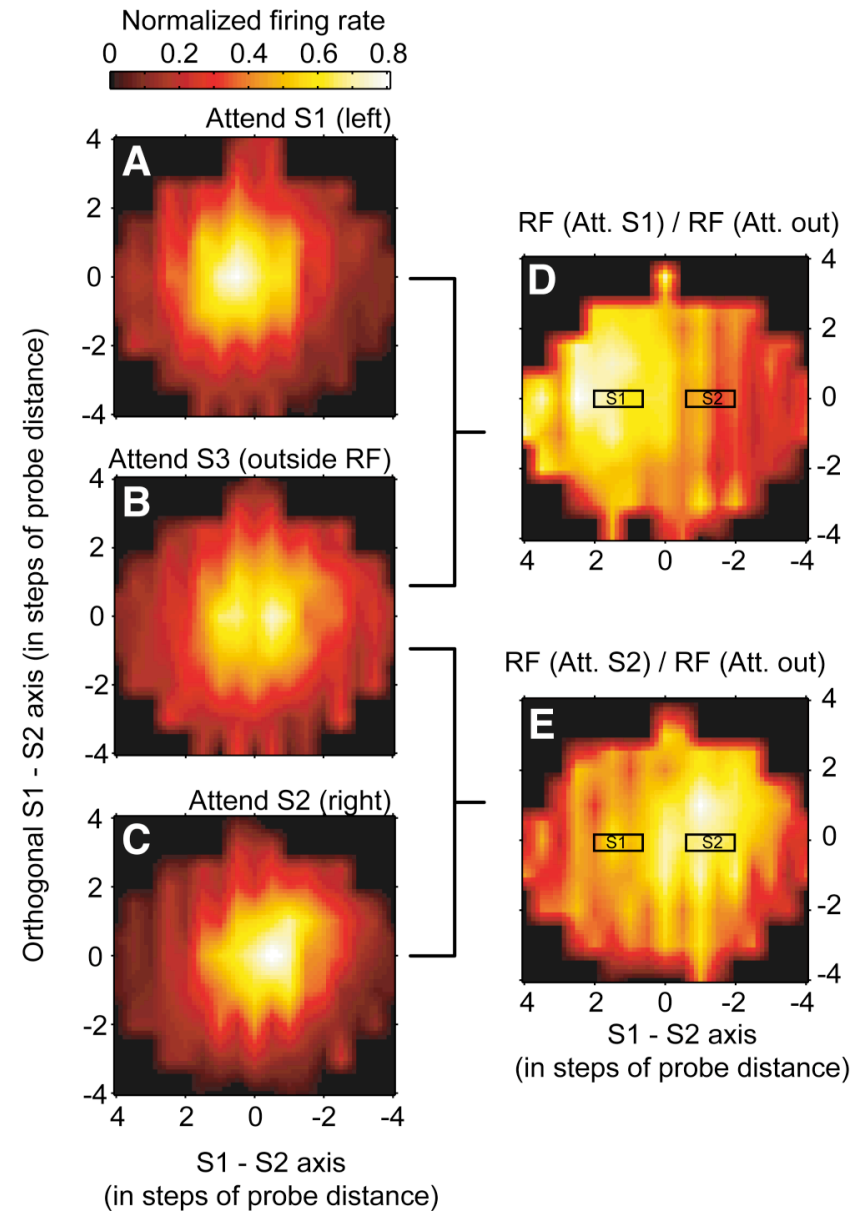

Figure 6: Average profiles of the non-parametric interpolated RF profiles of the three attentional conditions and derivation of an average, normalized attentional profile. A-C: Average RF when attention was directed to stimulus $S 1$ inside the RF ( $A$ : to the left of $0 / 0)$, to stimulus S3 outside the RF (B), and to stimulus $S 2$ inside the RF ( $C$, to the right of $0 / 0)$. D$E$ : Average attentional effect illustrated as the ratio of the RF profiles of attend-S1-to-attend-S3 (D) and attend-S2-to-attend-S3 $(E)$. The relative positions of stimulus $\mathrm{S} 1$ and $\mathrm{S} 2$ in the attentional maps are indicated by black rectangles (at positions of $\pm 1, \pm 1.5$, or 2 probe 'steps' across cells, varying as a function of the chosen mapping grid for individual neuronal RFs). Maps were smoothed by linearly interpolation between adjacent data points in the plot. the significant relation of attentional spread and eccentricity also holds for the whole sample of fitted data points $(r=0.50, p<0.001$, red plus grey data points in fig. 5D).

To investigate a possible influence of receptive field size on the correlation of the estimated attentional spread with eccentricity we analyzed the ratio of attentional spread to receptive field size as a function of eccentricity. We found a constant ratio (fig. $5 \mathrm{E}$, correlation $r=0.13$, $p=0.276$; linear regression: intercept $=1.7$, slope $=0.0$ ). This result indicates that the attentional spotlight grows along with RF size across increasing eccentricity.

Moreover, this finding justifies to normalize RF maps with different RF size and from different eccentricities to derive a nonparametric estimation of the average attentional spotlight. A non-parametric estimation of the attentional profile and its relation to RF size could provide additional support for the described modeling approach. 
Therefore, the individual RF profiles were rotated to have the abscissa along the S1-S2 axis and origin $(0,0)$ at the center between the two stimuli S1 and S2. Then, the distance between adjacent probes along the S1-S2 axis were normalized to a unit grid step size. The resulting average RF maps for each attention condition (fig. $6 \mathrm{~A}-\mathrm{C}$ ) visualize our main finding that attention shifted RFs toward the attended stimulus, with the average RF in the attend-outside condition well centered at the origin of the axes. According to the model attention is acting multiplicatively on this RF profile in the attend outside condition. We can therefore estimate from these non-parametric average RF maps the average, normalized width of the attentional profile by taking the ratio of the RFs in the attend-inside to attend-outside condition. The resulting ratio maps are shown in fig. 6D-E and demonstrate an average attentional profile that is centered ('focused') around the position of the corresponding attended stimuli (S1 / S2). Importantly, the average size of the attentional profile in the ratio maps (square root of the area above half maximum) of 5.2 (attend S1 versus S3: 5.1; attend S2 versus S3: 5.3) was about $60 \%$ bigger then the size of the average RF maps from the individual attention conditions (attend S1, S2 and S3: 3.2, 3.3, and 3.6). Thus, the results from this nonparametric analysis correspond closely to the average prediction of the parametric model, providing a very similar estimation of the attentional spread (relative to the RF size) underlying the RF shift and shrinkage.

\section{Discussion}

Our results document a correlation between the shift of MT receptive field centers and the concurrent shrinkage of their size when attention is relocated from a location far outside to a stimulus inside of the receptive field. This finding was evident in a non-parametric estimation of receptive field parameters as well as for a conservative selection of those receptive fields well fit by a bell-shaped (Gaussian) profile. We demonstrate how the non-multiplicative effect of attention on position and size of MT receptive fields can be quantitatively accounted for by a general gain mechanism modulating the spatial weighting of sensory inputs to area MT neurons and assuming a bell shape spatial profile. The proposed gain model also allowed us to estimate the size of the "attentional spotlight" as to be approximately 1.6 times larger than the receptive fields in MT and independent of eccentricity and this finding helped to visualize the attentional profile acting on the RF inputs. This independency suggests that the minimal size of the attentional spotlight reflects the spatial spread of the feed-forward input to area MT at a given eccentricity rather than being able to be adapted to stimuli smaller than the receptive field.

When attention is directed to a subregion of the receptive field of MT neurons the response to probe stimuli close to the attentional focus is enhanced while the 
response to distant probes is reduced, i.e. the receptive field of these neurons shift towards the attended position. This attentional attraction of spatial sensitivity reflects a non-multiplicative change of a neuron's spatial tuning curve. When attention is shifted between inside and outside the receptive field we observed only a small (5\%) non-significant change in the receptive field. While this modulation is smaller than the approximately $10 \%$ response modulation by spatial attention that was observed in MT in a previous study (Treue and Martinez-Trujillo, 1999), this is most likely due to the use of the late, sustained response to stimuli in the previous study, as attentional effects are known to be weak for the early components of neuronal responses to the appearance of stimuli and grow in the course of a trial when using hazard rates with a positive slope (Motter, 1994; Roelfsema, Lamme and Spekreijse, 1998; Reynolds, Chelazzi and Desimone, 1999; Treue and Maunsell, 1999; McAdams and Maunsell, 1999; Ghose and Maunsell, 2002).

Previous studies have shown response gain modulation when switching spatial attention from outside to inside a receptive field. This multiplicative modulation does not affect tuning preference or selectivity to features like orientation and direction of motion in extrastriate visual areas like MT and V4 (McAdams and Maunsell, 1999; Treue and Martinez-Trujillo, 1999; Martinez-Trujillo and Treue, 2004). These observations stand in marked contrast to the highly nonmultiplicative attentional modulation of spatial tuning that we observed. We propose a gain model that resolves this apparent contradiction by suggesting that the attentional modulation of receptive fields in area MT is the result of a bell shaped pattern of multiplicative modulation imposed onto the input to MT neurons. The model provides a good account of the data and thus shows how multiplicative neuronal interactions can create non-multiplicative effects.

This push-pull effect of attention on spatial tuning resembles the sharpening of direction tuning across the population of MT neurons reported previously (Martinez-Trujillo and Treue, 2004). That study reported a response increase for those neurons preferring directions close to the attended direction of motion and a response suppression for neurons tuned for non-attended directions in line with the feature-similarity gain model of attention (Treue and Martinez-Trujillo, 1999). Despite these similarities it should be pointed out that the two effects differ in critical aspects. For one in the population response to direction of motion MartinezTrujillo and Treue observed a linear decline of the multiplicative gain factor with increasing dissimilarity of the attended to the preferred direction of motion while we report here that attentional modulation of the spatial tuning is brought about by a bell-shaped (e.g. Gaussian) spatial gain pattern. This difference in the modulation of spatial and feature selective responses is not surprising given the different characteristics of spatial and feature tuning: Tuning selectivity for the direction of motion is similar in area MT and its input areas, indicating that MT 
neurons pool across input neurons with the same preferred direction. A featuresimilarity gain modulation (Treue and Martinez-Trujillo, 1999) can therefore be achieved by changing the overall gain of MT neurons directly or using the same factor across all its inputs. Spatial tuning on the other hand is far broader in MT than in its input areas. It is therefore created de novo from inputs to MT that differ in their preferred location and this integration process can be specifically targeted by attentional mechanisms. This could be achieved with at least two approaches: The neurons in an earlier area (e.g. V1) with smaller receptive fields that provide the input to one MT neuron might already be multiplicatively modulated by attention. Alternatively, the receptive field shift and shrinkage could be due a specific pattern of multiplicative attentional modulations imposed onto the synaptic inputs of an MT neuron. In both cases, spatial attention would reshape the spatial sensitivity of MT neurons in a multiplicative way but with a gain that is locally adjusted according to a bell-shaped spatial weighting function (e.g. a Gaussian).

The mechanistic explanation of the proposed multiplicative attentional gain model is closely related to the framework of gain fields that has been applied to neuronal responses throughout sensory cortex (Andersen and Essick, 1985; Salinas and Abbott, 1996, 2000, 2001, 2004). Gain field architectures describe neuronal response characteristics to a sensory stimulus as an interaction with additional parameters such as, for example, eye position in the orbit and gaze direction (Brotchie et al., 1995), or describe a multiplicative interaction of different stimulus attributes (Pena and Konishi, 2001, 2004). Similar to gain fields our proposed multiplicative model provides a general formalization of how the integration of sensory inputs is modulated by feedback connections (Salinas and Abbott, 1997; see also: Olshausen et al., 1993). In the existing approaches the multiplicative mechanisms had to be inferred from neural network simulations. In contrast, we provide a straightforward mathematical formalization of the model that allows investigating the integration of neuronal responses across visual cortex without taking recourse to simulations.

One notable prediction of the model is a broad spatial spread of the attentional influence underlying the observed modulation of spatial sensitivity in area MT. The estimated spread of the attentional influence was about 2.2 degrees at the fovea, increasing systematically by about two degrees every degree of eccentricity. Such a broad influence resonates with psychophysical studies that have proposed a coarse resolution of attention and an increase of the size of the 'spotlight of attention' with eccentricity (Suzuki and Cavanagh, 1997; Intriligator and Cavanagh, 2001; Cutzu and Tsotsos, 2003; Tse et al., 2003). This finding could also explain that attention to regions just outside the receptive field of area V4 neurons in the temporal visual pathway induces changes in the spatial sensitivity similar to the changes with attention inside the receptive field we report (Connor et al., 1997). 
The proposed feed-forward architecture is not the sole candidate to account for the observed neurophysiological findings. Previous models of selective attentional modulation in sensory cortex have emphasized the modulation of recurrent or lateral inhibitory interactions to enhance filtering of distracting visual information from access to higher visual area (e.g. Olshausen etal., 1993; Tsotsos et al., 1995; Reynolds and Desimone, 1999; Deco and Schuermann, 2000; Compte and Wang, 2006; Hamker and Zirnsak, 2006). However, the proposed model of multiplicative interactions of attention and sensory responses reflects a particularly parsimonious and well-formalized account of neuronal changes under different attentional conditions, without the need of assuming an attentional effect directly targeting inhibitory interactions among neurons.

The proposed gain model of attention suggests that multiplicative operations could reflect a general computational mechanism underlying selective modulation of basic sensory responses of neurons. This suggestion is supported by previous studies showing that single neurons are capable to combine excitatory and inhibitory inputs about sensory attributes multiplicatively (Gabbiani et al., 2002), and that multiplicative gain is capable to control the responsiveness of cortical neurons (Chance and Abbott, 2002).

In summary, our observation of neuronal receptive field shifts toward and shrinkage around the focus of attention suggest that spatial attention acts like an attractor of spatial sensitivity. This non-multiplicative modulation of the spatial tuning characteristics of single neurons is consistent with a general multiplicative modulation impinging on the input of these neurons. Such an attentional gain has also been shown during feature-based attention (McAdams and Maunsell, 1999; Martinez-Trujillo and Treue, 1999, 2004) suggesting that both, space-based and feature-based attentional selection of sensory inputs utilizes multiplicative modulations to create complex changes in the responses of neurons in extrastriate cortex. 


\section{References}

Andersen RA, Essick GK, Siegel RM (1985) Encoding of spatial location by posterior parietal neurons. Science 230:456-458.

Bichot NP, Rossi AF, Desimone R (2005) Parallel and serial neural mechanisms for visual search in macaque area V4. Science 308:529-534.

Britten KH, Heuer HW (1999) Spatial summation in the receptive fields of MT neurons. J Neurosci 19:5074-5084.

Brotchie PR, Andersen RA, Snyder LH, Goodman SJ (1995) Head position signals used by parietal neurons to encode locations of visual stimuli. Nature 375:232-235.

Carrasco M, Ling S, Read S (2004) Attention alters appearance. Nat. Neurosci. 7:308-313

Chance FS, Abbott LF, Reyes AD (2002) Gain modulation from background synaptic input. Neuron 35:773-782.

Chelazzi L, Miller EK, Duncan J, Desimone R (1993) A neural basis for visual search in inferior temporal cortex. Nature 363:345-347.

Compte A, Wang XJ (2006) Tuning curve shift by attention modulation in cortical neurons: a computational study of its mechanisms. Cereb Cortex 16:761778.

Connor CE, Preddie DC, Gallant JL, Van Essen DC (1997) Spatial attention effects in macaque area V4. J Neurosci 17:3201-3214.

Cook EP, Maunsell JH (2002) Dynamics of neuronal responses in macaque MT and VIP during motion detection. Nat Neurosci 5:985-994.

Croner LJ, Kaplan E (1995) Receptive fields of P and M ganglion cells across the primate retina. Vision Res 35:7-24.

Cutzu F, Tsotsos JK (2003) The selective tuning model of attention: psychophysical evidence for a suppressive annulus around an attended item. Vision Res 43:205-219.

Deco G, Schuermann B (2000) A hierarchical neural system with attentional topdown enhancement of the spatial resolution for object recognition. Vision Res 40:2845-2859.

Gabbiani F, Krapp HG, Koch C, Laurent G (2002) Multiplicative computation in a visual neuron sensitive to looming. Nature 420:320-324.

Ghose GM, Maunsell, JHR (2002) Attentional modulation in visual cortex depends on task timing. Nature 419:616-620.

Hamker FH, Zirnsak M (2006) V4 receptive field dynamics as predicted by a systems-level model of visual attention using feedback from the frontal eye field. Neural Netw 19:1371-1382.

Itti L, Koch C (2001) Computational modelling of visual attention. Nat Rev Neurosci 2:194-203. 
Luck SJ, Chelazzi L, Hillyard SA, Desimone R (1997) Neural mechanisms of spatial selective attention in areas $\mathrm{V} 1, \mathrm{~V} 2$, and $\mathrm{V} 4$ of macaque visual cortex. J Neurophysiol 77:24-42.

Martinez-Trujillo J, Treue S (2002) Attentional modulation strength in cortical area MT depends on stimulus contrast. Neuron 35:365-370.

Martinez-Trujillo JC, Treue S (2004) Feature-based attention increases the selectivity of population responses in primate visual cortex. Curr Biol 14:744-751.

Maunsell JHR, Treue S (2006) Feature-based attention in visual cortex. Trends Neurosci 29:317-322.

McAdams CJ, Maunsell JHR (1999) Effects of attention on orientation-tuning functions of single neurons in macaque cortical area V4. J Neurosci 19:431441.

McAdams CJ, Maunsell JH (1999) Effects of attention on the reliability of individual neurons in monkey visual cortex. Neuron 23:765-773.

McAdams, CJ, Maunsell, JHR (2000). Attention to both space and feature modulates neuronal responses in macaque area V4. J. Neurophysiol. 83:1751-1755.

Moran J, Desimone R (1985) Selective attention gates visual processing in the extrastriate cortex. Science 229:782-784.

Motter BC (1994) Neural correlates of attentive selection for color or luminance in extrastriate area V4. J Neurosci 14:2178-2189.

Olshausen BA, Anderson CH, Van Essen DC (1993) A neurobiological model of visual attention and invariant pattern recognition based on dynamic routing of information. J Neurosci 13:4700-4719.

Pena JL, Konishi M (2001) Auditory spatial receptive fields created by multiplication. Science 292:249-252.

Pena JL, Konishi M (2004) Robustness of multiplicative processes in auditory spatial tuning. J Neurosci 24:8907-8910.

Raiguel S, Van Hulle MM, Xiao DK, Marcar VL, Orban GA (1995) Shape and spatial distribution of receptive fields and antagonistic motion surrounds in the middle temporal area (V5) of the macaque. Eur J Neurosci 7:20642082.

Recanzone GH, Wurtz RH (2000) Effects of attention on MT and MST neuronal activity during pursuit initiation. J Neurophysiol 83:777-790.

Reynolds JH, Desimone R (1999) The role of neural mechanisms of attention in solving the binding problem. Neuron 24:19-29, 111-125.

Reynolds JH, Chelazzi L (2004) Attentional modulation of visual processing. Annu Rev Neurosci 27:611-647.

Reynolds JH, Chelazzi L, Desimone R (1999) Competitive mechanisms subserve attention in macaque areas V2 and V4. J Neurosci 19:1736-1753.

Reynolds JH, Pasternak T, Desimone R (2000) Attention increases sensitivity of V4 neurons. Neuron 26:703-714. 
Roelfsema PR, Lamme VA, Spekreijse H (1998) Object-based attention in the primary visual cortex of the macaque monkey. Nature 395:376-381.

Salinas E (2004) Fast remapping of sensory stimuli onto motor actions on the basis of contextual modulation. J Neurosci 24:1113-1118.

Salinas E, Abbott LF (1996) A model of multiplicative neural responses in parietal cortex. Proc Natl Acad Sci U S A 93:11956-11961.

Salinas E, Abbott LF (1997) Invariant visual responses from attentional gain fields. J Neurophysiol 77:3267-3272.

Salinas E, Thier P (2000) Gain modulation: a major computational principle of the central nervous system. Neuron 27:15-21.

Salinas E, Abbott LF (2001) Coordinate transformations in the visual system: how to generate gain fields and what to compute with them. Prog Brain Res 130:175-190.

Suzuki S, Cavanagh P (1997) Focused attention distorts visual space: an attentional repulsion effect. J Exp Psychol Hum Percept Perform 23:443463.

Treue S (2001) Neural correlates of attention in primate visual cortex. Trends Neurosci 24:295-300.

Treue S (2003a) Visual attention: the where, what, how and why of saliency. Curr Opin Neurobiol 13:428-432.

Treue S (2003b) Climbing the cortical ladder from sensation to perception. Trends Cogn Sci 7:469-471.

Treue S, Maunsell JH (1996) Attentional modulation of visual motion processing in cortical areas MT and MST. Nature 382:539-541.

Treue S, Maunsell JH (1999) Effects of attention on the processing of motion in macaque middle temporal and medial superior temporal visual cortical areas. J Neurosci 19:7591-7602.

Treue S, Martinez-Trujillo JC (1999) Feature-based attention influences motion processing gain in macaque visual cortex. Nature 399:575-579.

Tse PU, Sheinberg DL, Logothetis NK (2003) Attentional enhancement opposite a peripheral flash revealed using change blindness. Psychol Sci 14:91-99.

Tsotsos JK, Culhane WYK, Wai Y, Lai N, Davis N, Nuflo F (1995) Modeling visual attention via selective tuning. Artificial Intelligence 78:507-545.

Williford T, Maunsell JH (2006) Effects of spatial attention on contrast response functions in macaque area V4. J Neurophysiol 96:40-54.

Womelsdorf T, Anton-Erxleben K, Pieper F, Treue S (2006) Dynamic shifts of visual receptive fields in cortical area MT by spatial attention. Nat Neurosci 9:1156-1160. 
Supplementary Material for

\title{
RECEPTIVE FIELD SHIFT AND SHRINKAGE IN MACAQUE AREA MT THROUGH ATTENTIONAL GAIN MODULATION
}

\author{
Womelsdorf, T., Tzvetanov, T., Anton-Erxleben, K., Treue, S.
}

\section{Model derivation of equations 3-6 (main text)}

Here we describe in more detail the mathematical steps for obtaining equations 36 of the model.

The receptive field of a neuron centred at $x_{R}$, width $\sigma_{R}$, and maximal firing rate of $A_{R}$ is defined as:

$$
R F(x)=A_{R} \exp \left(-\frac{\left(x-x_{R}\right)^{2}}{2\left(\sigma_{R}\right)^{2}}\right)
$$

The attentional spatial factor, centred at $x_{A t t}$, width $\sigma_{A t t}$, and attentional amplitude $A_{A t}$, is defined as:

$$
A(x)=1+A_{A t t} \exp \left(-\frac{\left(x-x_{A t t}\right)^{2}}{2\left(\sigma_{A t t}\right)^{2}}\right)
$$

By multiplying the two terms one obtains:

$$
R F^{\text {attended }}(x)=R F(x)+A_{R} \exp \left(-\frac{\left(x-x_{R}\right)^{2}}{2\left(\sigma_{R}\right)^{2}}\right) \times A_{A t t} \exp \left(-\frac{\left(x-x_{A t t}\right)^{2}}{2\left(\sigma_{A t t}\right)^{2}}\right)
$$

where the second term can be rewritten as:

$$
A_{R} A_{A t t} \exp \left(-\frac{\left(\sigma_{A t t}\right)^{2}\left(x-x_{R}\right)^{2}+\left(\sigma_{R}\right)^{2}\left(x-x_{A t t}\right)^{2}}{2\left(\sigma_{R}\right)^{2}\left(\sigma_{A t t}\right)^{2}}\right)
$$

The sum of the two second-order polynomials in the exponential can be rearranged such that only one second-order polynomial contains the variable spatial position $(x)$. This gives the following expression: 


$$
A_{R} A_{A t t} \exp \left(-\frac{1}{2}\left(\frac{1}{\left(\sigma_{R}\right)^{2}}+\frac{1}{\left(\sigma_{A t t}\right)^{2}}\right)\left[x-\frac{\frac{x_{R}}{\left(\sigma_{R}\right)^{2}}+\frac{x_{A t t}}{\left(\sigma_{A t t}\right)^{2}}}{\frac{1}{\left(\sigma_{R}\right)^{2}}+\frac{1}{\left(\sigma_{A t t}\right)^{2}}}\right]^{2}\right) \times \exp \left(-\frac{1}{2} \frac{1}{\frac{1}{\left(\sigma_{R}\right)^{2}}+\frac{1}{\left(\sigma_{A t t}\right)^{2}}}\left(x_{R}-x_{A t t}\right)^{2}\right)
$$

The second exponential term is clearly independent of the spatial position $x$, and depends only on the relative distance between the initial receptive field center and the attentional center, and also on the initial receptive field and attentional sizes. Therefore, we can label it as one supplementary amplitude, $A^{\text {attended. }}$. In the first Gaussian function, we can define a new width and a new center. Then one obtains equations (3-6).

\section{Normalization of receptive field profile volume: Shrinkage versus amplitude changes}

In the main text we state that the model does not make any prediction on the amplitude of the attention modulated receptive field profile. Here, we explain why the amplitude of the receptive field profile cannot be used as a measure of the influence of spatial attention.

The model prediction assumes that the attentional modulation of the receptive field profile can be only seen in the position and size parameters. The final maximal firing rate of the neuron (the receptive field amplitude) cannot be used as a sensitive measure, because it is influenced by cortical normalization processes (e.g. Croner \& Kaplan, 1995). These normalization processes tend to keep the total volume under the receptive field constant, which increases the amplitude when the receptive field is getting smaller (i.e. it creates a negative correlation between receptive field size and amplitude). This prediction is supported by our data (suppl. fig. 1): With a decrease of receptive field size with attention (the ratio size RFin / size RFout below 100\%; y-axis) the amplitude of the RFin profile tends to be higher than for the RFout profile (ratio amplitude RFin / amplitude RFout above 1.0; $\mathrm{x}$-axis). This effect was statistically confirmed through a significant negative correlation of the two measures: in the analysis based on the receptive field parameters extracted from Gaussian fits (suppl. fig 1A; $r=-0.27, p<0.01, n=$ $97)$, as well as in the non-parametric analysis based on spline-interpolated receptive field profiles (suppl. fig. $1 \mathrm{~B} ; r=-0.43, p<0.01$ ). Moreover, the amount of receptive field shift toward the attended position was not correlated to amplitude changes ( $r=0.03$, n.s., $n=97$ ) lending additional support that amplitude normalization follows and adapts to the RF input size change. 


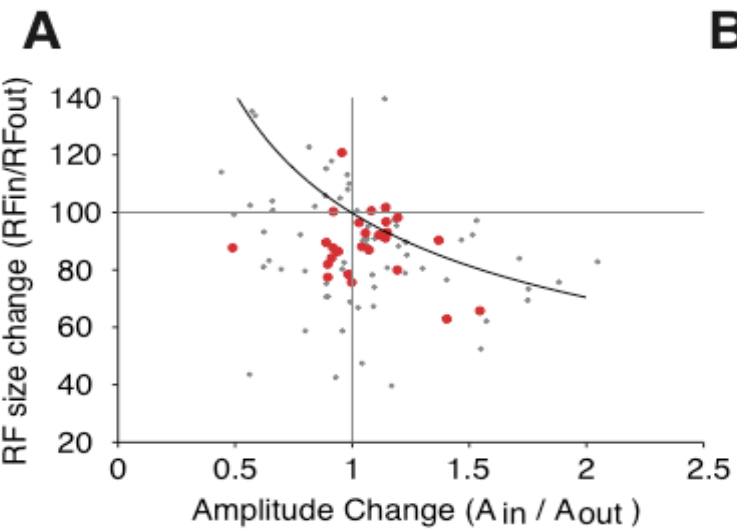

B

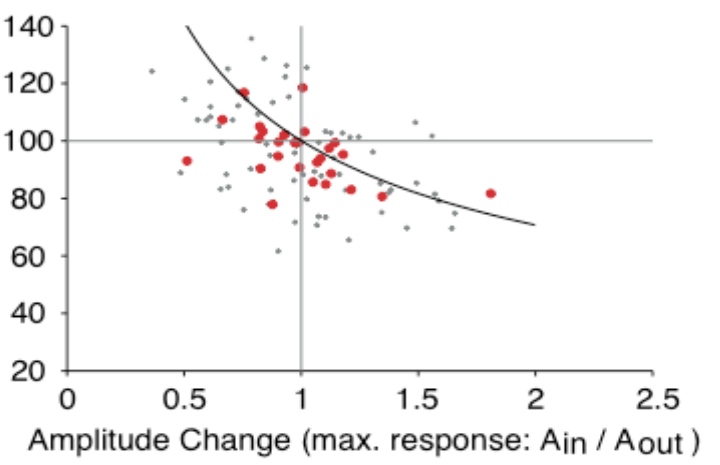

Supplemental figure 1: Relationship between size changes and amplitude changes of the receptive fields caused by the allocation of spatial attention. A: Receptive field size and amplitude changes extracted from the parametric analysis of the receptive fields (based on Gaussian-fitted receptive field profiles). Receptive field shrinkage is negatively correlated to the amplitude changes ( $r=-0.27$, $\mathrm{p}<0.01, \mathrm{n}=97$ ). B: Receptive field size and maximum amplitude changes extracted from the nonparametric analysis (based on spline-interpolated receptive field profiles). Shrinkage and amplitude changes are significantly correlated $(r=-0.43, p<0.01, n=97)$. In both panels, when spatial attention reduces receptive field size ( $y$-axis values below $100 \%$ ) the maximal firing rate of the neuron tends to increase ( $x$-axis values above 1.0 ), which is expected from a normalization process that keeps the volume under the receptive field response surface constant. To illustrate this the black solid curve in each panel is the prediction when assuming a full normalization. 


\subsection{Attention changes perceived size of moving visual patterns}

Assuming that retinotopic maps of spatially restricted receptive fields enable the visual system to construct a representation of visual space, using a labeled-line principle, shifts of receptive field locations without updating their position label should lead to systematic distortions in the perception of spatial relations. Here, we show that attention indeed induces such distortions by demonstrating that attention increases perceived stimulus size. 



\section{Attention changes perceived size of moving visual patterns}

Katharina Anton-Erxleben

\author{
Christian Henrich
}

Cognitive Neuroscience Laboratory,

German Primate Center, Goettingen, Germany

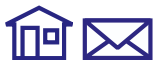

Universität Karlsruhe, Fakultät für Informatik, IAKS, Karlsruhe, Germany

Spatial attention shifts receptive fields in monkey extrastriate visual cortex toward the focus of attention (S. Ben Hamed, J. R. Duhamel, F. Bremmer, \& W. Graf, 2002; C. E. Connor, J. L. Gallant, D. C. Preddie, \& D. C. Van Essen, 1996; C. E. Connor, D. C. Preddie, J. L. Gallant, \& D. C. Van Essen, 1997; T. Womelsdorf, K. Anton-Erxleben, F. Pieper, \& S. Treue, 2006). This distortion in the retinotopic distribution of receptive fields might cause distortions in spatial perception such as an increase of the perceived size of attended stimuli. Here we test for such an effect in human subjects by measuring the point of subjective equality (PSE) for the perceived size of a neutral and an attended stimulus when drawing automatic attention to one of two spatial locations. We found a significant increase in perceived size of attended stimuli. Depending on the absolute stimulus size, this effect ranged from $4 \%$ to $12 \%$ and was more pronounced for smaller than for larger stimuli. In our experimental design, an attentional effect on task difficulty or a cue bias might influence the PSE measure. We performed control experiments and indeed found such effects, but they could only account for part of the observed results. Our findings demonstrate that the allocation of transient spatial attention onto a visual stimulus increases its perceived size and additionally biases subjects to select this stimulus for a perceptual judgment.

Keywords: attention, appearance, illusion, psychophysics, receptive field

Citation: Anton-Erxleben, K., Henrich, C., \& Treue, S. (2007). Attention changes perceived size of moving visual patterns. Journal of Vision, 7(11):5, 1-9, http://journalofvision.org/7/11/5/, doi:10.1167/7.11.5.

\section{Introduction}

\section{Attention alters appearance}

Perception is not an objective representation of the sensory input, but rather results from an interaction of bottom-up sensory information with top-down influences. Attention is the central top-down mechanism for selecting relevant aspects of the visual scene for preferred processing. This deployment of spatial attention not only results in lowered thresholds, faster reaction times, better spatial resolution, and more accurate performance (for example, see Dobkins \& Bosworth, 2001; Posner, 1980; Sperling \& Dosher, 1986; Yeshurun \& Carrasco, 1998), but also in an altered subjective perception of appearance: Attention has been found to increase apparent contrast (Carrasco, Ling, \& Read, 2004), spatial frequency, gap size (Gobell \& Carrasco, 2005), motion coherence (Liu, Fuller, \& Carrasco, 2006), color saturation (Fuller \& Carrasco, 2006), flicker rate (Montagna \& Carrasco, 2006), and perceived speed (Turatto, Vescovi, \& Valsecchi, 2007).
Thus, attention not only enhances perception, it also distorts our representation of the visual scene according to the behavioral relevance of its components.

\section{Perception of space}

Striate cortex and many extrastriate cortical areas represent the spatial layout of our visual environment in a retinotopic map of spatially restricted receptive fields. The relative response strength of different neurons that represent different spatial locations enables the visual system to construct a representation of visual space. The extraction of spatial location from such a population activity requires a labeled-line principle; that is, the location of any neuron's receptive field needs to be known to the decoder.

\section{Dynamic receptive fields and functional consequences}

Receptive fields are not static, however, as has been demonstrated for several areas throughout the visual 
system. In the lateral intraparietal (LIP) area, receptive field size and position change in the context of a saccade (Kusunoki \& Goldberg, 2003) and also as a function of behavioral state: Receptive fields measured during attentive fixation are more foveal, consistent with a shift of receptive fields toward the spatial focus of attention (Ben Hamed, Duhamel, Bremmer, \& Graf, 2002). In extrastriate area V4, receptive field profiles are shifted toward an attended stimulus (Connor, Gallant, Preddie, \& Van Essen, 1996; Connor, Preddie, Gallant, \& Van Essen, 1997). This has also been documented recently for neurons in the medial temporal area (MT), which is part of the dorsal visual pathway and is essential for processing visual motion: Spatial attention attracts $\mathrm{MT}$ receptive fields toward the spatial focus of attention and thereby changes the spatial position most effective in stimulating a given neuron. The shift is most pronounced for receptive fields overlapping the spatial focus of attention and is reduced for receptive fields further away from the attentional focus (Womelsdorf, Anton-Erxleben, Pieper, \& Treue, 2006). This will concentrate neuronal resources in an attended spot for enhanced processing, but in addition receptive field shifts might influence those aspects of spatial perception that depend on an accurate decoding of receptive field locations, such as the sizes of objects or spatial relations between objects.

\section{Hypothesis}

If the position label of a neuron is not updated when the receptive field center shifts, the position of a stimulus might be misperceived. Specifically, receptive fields centered outside the edges of an object would, when attracted toward the object's center, report the edge as lying within the receptive field, perceptually enlarging the object.

Adapting a design introduced by Carrasco et al. (2004) for showing attentional modulation of subjective appearance, we tested if drawing automatic attention to a stimulus increases its apparent size.

\section{Methods}

\section{General design}

The general design was adapted from Carrasco et al. (2004): While subjects had to maintain fixation, a cue was briefly $(71 \mathrm{~ms})$ presented either peripherally or at the fixation point to automatically attract attention to the respective location; then two differently sized moving random dot patterns (RDPs) were presented. They were centered left and right of the fixation point at the same eccentricity as the cue (Figure 1). Note that our hypothesis required this alignment of cue and stimulus, whereas in

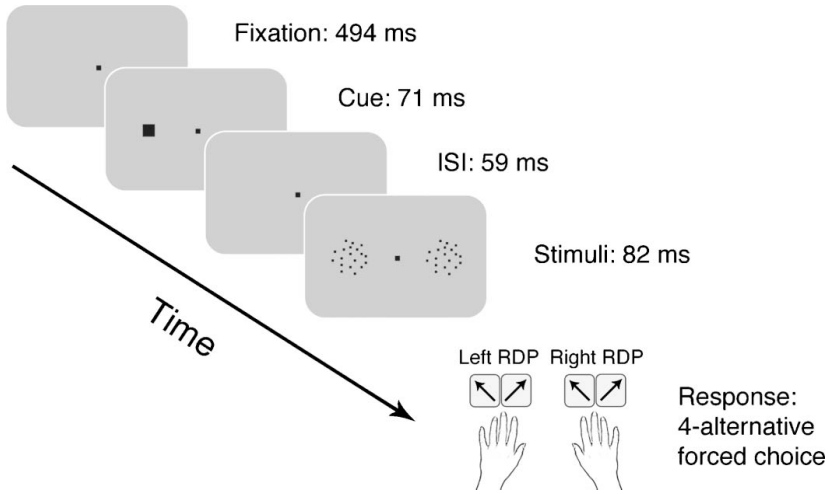

Figure 1. Task design. After a fixation period of $494 \mathrm{~ms}$, a cue was flashed for $71 \mathrm{~ms}$ at $4^{\circ}$ eccentricity left or right of the fixation point (peripheral cue) or exactly at the fixation point (neutral cue). Then followed a 59-ms interstimulus interval (ISI) and then test and standard RDPs were presented also at $4^{\circ}$ eccentricity left or right of the fixation point for $82 \mathrm{~ms}$. In all experiments, test diameter varied from $80 \%$ to $120 \%$ of the standard diameter in nine equidistant steps. In the main experiment, subjects were instructed to report the horizontal motion component (left or right) of the larger RDP by pressing one of four keys (4-alternative forced choice design).

the study of Carrasco et al. the cue was slightly offset from the stimulus location. Subjects were instructed to report if the larger of the two patterns moved with a rightward or leftward tilt from vertical. The timing of cue and target was such that subjects' automatic attention was maximally allocated on the cued RDP at the time they had to perform the size judgment: This design enabled us to measure subjective appearance of the size of the attended and the unattended pattern without equally distributing attention across both of them. The short presentation times also prevent eye movements as the interval between cue onset and stimulus offset is shorter than typical saccadic latency (Bichot, Thompson, Rao, \& Schall, 2001; Mayfrank, Kimmig, \& Fischer, 1987).

We used moving stimuli in our experiments because receptive field changes with attention have been investigated in area MT in the motion processing system (Womelsdorf et al. 2006). Given that receptive field shifts have also been shown in other cortical areas (Ben Hamed et al., 2002; Connor et al., 1996, 1997), our hypothesis of an increase in perceived size can also be applied to stationary stimuli.

\section{Experimental setup and procedure}

Experiments were performed in a dimly lit experimental cabin. Subjects used a chin rest positioned $57 \mathrm{~cm}$ from a CRT monitor on which the stimuli were presented (LaCie electron22blue IV 22-in. CRT) with a viewable area of $40^{\circ} \times 30^{\circ}$, a resolution of 40 pixels $/ \mathrm{deg}$, and a refresh rate 
of $85 \mathrm{~Hz}$. Stimuli were presented on a gray background $\left(34.2 \mathrm{~cd} / \mathrm{m}^{2}\right)$. All stimuli were black $\left(0.1 \mathrm{~cd} / \mathrm{m}^{2}\right)$. Stimulus presentation and recording of the subjects' responses were controlled by a custom software developed in-house, which was run on an Apple Macintosh computer.

Subjects initiated each trial by pressing the space bar on a computer keyboard. Thirteen subjects (six male, seven female) participated in all experiments. All were students between 19 and 26 years, all were naive to the purpose of the experiments and had normal or corrected-to-normal vision. Within one experimental session, subjects first performed 500 trials of a tuning measurement to adjust the difficulty of the task and then one of six different size experiments (1000 trials each) described below. All six experiments were performed by each subject in six sessions in randomized order.

\section{Tuning experiment}

Because the allocation of attention is known to vary with task difficulty (Spitzer \& Richmond, 1991; Urbach \& Spitzer, 1995), we compensated for interindividual performance differences in the direction discrimination task as well as difficulty differences due to the different experimental settings, for example, standard size, or training effects during the course of the whole series of experiments. To ensure that the size perception experiments were performed under comparable conditions, we varied the deviation of motion direction from the vertical. The directions used in each experiment were adjusted for each subject by preceding each experimental run with a "tuning" measurement. Subjects were asked to maintain fixation on the fixation point, a small black square $\left(0.2^{\circ} \times 0.2^{\circ}\right)$. Immediately after the start of the trial, a black arrow $\left(0.4^{\circ}\right.$ long and $0.1^{\circ}$ wide) pointing either to the left or to the right was presented $0.5^{\circ}$ to the left or to the right of the fixation point for $306 \mathrm{~ms}$. Directly after arrow offset, two black RDPs with the same parameters as the standard stimulus of the following experiment were presented left and right of the fixation point. Both were either moving upward or downward with a leftward or rightward deviation ranging from $3^{\circ}$ to $15^{\circ}$ from vertical in five equidistant steps (with an exception: for the experiment with the largest standard size we used steps of $1^{\circ}, 2^{\circ}, 3^{\circ}, 6^{\circ}, 9^{\circ}$, and $12^{\circ}$ in the tuning measurement). Subjects were instructed to report the sidewise direction component (left or right) of the stimulus indicated by the arrow. For the following experiment, we chose the deviation from vertical for which the subject reached a performance of $75 \%$ correct.

\section{Main experiment: task and stimuli}

In the main experiment, each trial began with the presentation of the fixation point for $494 \mathrm{~ms}$. Then the cue, a black square $\left(0.25^{\circ} \times 0.25^{\circ}\right)$, was flashed for $71 \mathrm{~ms}$ either at $4^{\circ}$ to the left or to the right of the fixation point (peripheral cue) or on top of the fixation point (neutral cue). After an interstimulus interval (ISI) of $59 \mathrm{~ms}$, two circular RDPs were shown for $82 \mathrm{~ms}$, centered $4^{\circ}$ left and right of the fixation point. Both RDPs moved upward or downward with a leftward or rightward deviation from vertical. The RDP on one side was always the standard stimulus with a size of $2^{\circ}$ diameter, whereas the size of the other RDP (the test stimulus) varied from $80 \%$ to $120 \%$ of the standard diameter $\left(1.6-2.4^{\circ}\right)$ in nine equidistant steps. The positions of the cue, the test, and the standard stimulus as well as the motion direction of both stimuli (up or down with leftward or rightward tilt) were randomized. To determine the effect of absolute stimulus size, we also ran this experiment with standard stimuli of $1^{\circ}$ and $4^{\circ}$ diameter.

\section{Control experiments}

Several control experiments were conducted to investigate potential cue biases. In the first control experiment, the cue appeared after the stimuli (postcue), with the same cue and stimulus presentation durations and the same ISI, an approach also used by Gobell and Carrasco (2005). In the second control experiment, the same settings as in the main experiment were used but subjects were instructed to report the direction of motion of the smaller of the two RDPs (reversed instructions experiment; also used by Carrasco et al., 2004; Fuller \& Carrasco, 2006; Montagna \& Carrasco, 2006; Turatto et al., 2007). In the third control experiment, subjects were asked to report only which of the stimuli appeared smaller without performing the motion discrimination task (single task experiment, also used by Carrasco et al., 2004).

\section{Data analysis}

For each experiment, we determined the proportion of standard cued, test cued, and neutral cue trials in which subjects chose the test stimulus as the larger stimulus as a function of the test size (normalized to the respective standard size). These values were fitted with a psychometric function by an iterative likelihood maximization procedure, and the point of subjective equality (PSE), where subjects chose test and standard equally often, was determined for each cue condition and each experiment. To estimate the goodness of the fits, a bootstrapping was performed (Wichmann \& Hill, 2001): 10,000 runs with 1,000 trials each were simulated for each experimental condition and subject, using the fitted psychometric functions as the basis for the simulated data. For only 1 of the 234 ( 13 subjects $\times 6$ experiments $\times 3$ conditions $)$ measured psychometric functions, a statistically significant difference between simulated and measured data was 
found (alpha adjusted for multiple comparisons: .00022; overall significance level: .05), and we therefore did not exclude data on this basis. Two subjects were excluded because they did not reach a size discrimination performance of $84 \%$ in the main experiment. The PSE values of the remaining 11 subjects were compared with a repeated measures ANOVA using SPSS software (SPSS Inc., IL): Effects of the different standard sizes were compared with a $3 \times 3$ repeated measures ANOVA with the factors cue location and standard size, effects of the order of cue and stimulus presentation were compared with a $3 \times 2$ repeated measures ANOVA with the factors cue location and cue presentation time, and effects of the instruction (report direction of larger RDP, report direction of smaller RDP, only report smaller RDP) were compared with a $3 \times 3$ repeated measures ANOVA with the factors cue location and instruction. To analyze if the direction task difficulty was affected by the cue or the experimental condition, we compared direction discrimination performance for trials in which the cued stimulus was evaluated with trials in which there was a neutral cue and with trials in which the uncued stimulus was chosen, regardless if the chosen stimulus was test or standard with a $5 \times 3$ repeated measures ANOVA (experiment $\times$ cued/neutral/uncued chosen).

\section{Results}

In each experiment, we determined the PSE, that is, the test stimulus size which appears equal to the standard stimulus size, under different attentional conditions: standard stimulus cued, test stimulus cued, and neutral cue.

\section{Effects of attention on perceived size}

In Figure 2A, for each cue condition (standard stimulus cued, test stimulus cued, and neutral cue), the average proportion of trials in which subjects chose the test stimulus as larger than the standard stimulus is plotted as a function of test size relative to standard size. Data points were fitted with a psychometric function. The PSE corresponds to the $x$ value where the psychometric function crosses $50 \%$. In the neutral cue condition, this is a relative test size of almost exactly 1; that is, as expected in this condition the PSE and the point of physical equality (PPE) are equal. When the test stimulus is cued, the PSE is shifted to the left, indicating that a test stimulus which is $7 \%[ \pm 2 \%$ standard deviation $(S D)]$ smaller than the standard stimulus is perceived to be of equal size as the standard. When the standard stimulus is cued, the PSE is shifted $7 \%( \pm 2 \% S D)$ to the right, indicating that in this condition a test stimulus which is $7 \%( \pm 2 \% \quad S D)$ larger than the standard stimulus is
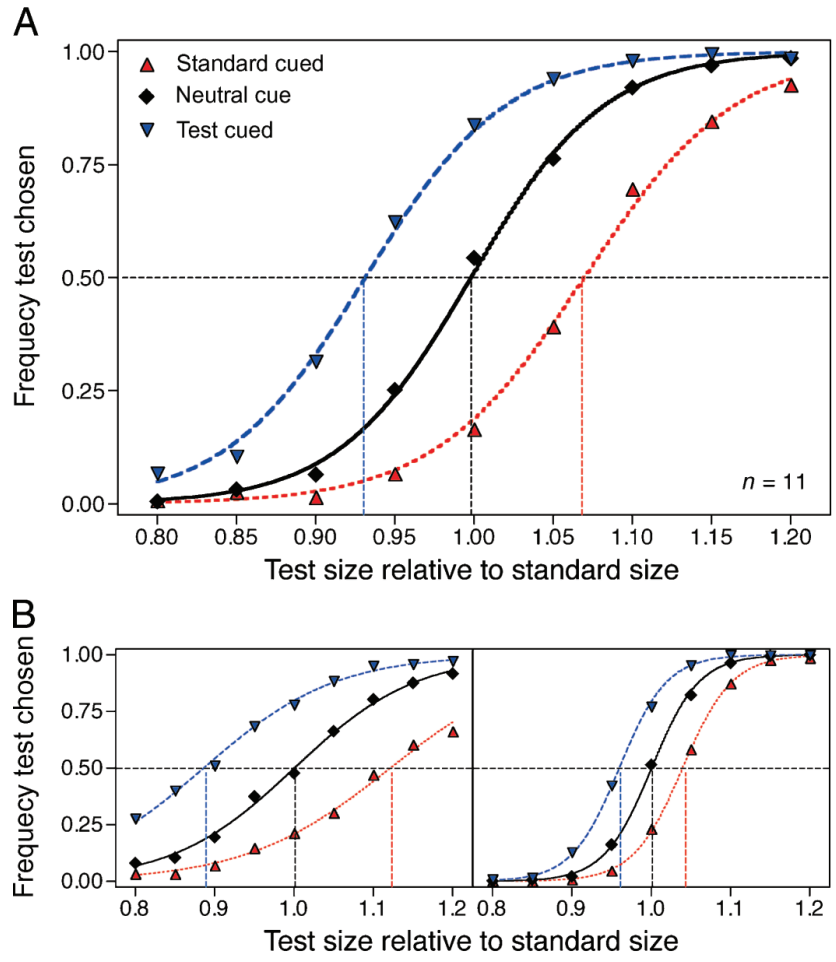

Figure 2. (A) Average results of the main experiment. For test cued, neutral, and standard cued trials, the percentage of trials is plotted in which the test stimulus was chosen as bigger than the standard stimulus of $2^{\circ}$ diameter as a function of test size. Data are averaged across 11 subjects and then fitted with a psychometric function using iterative likelihood maximization. (B) Average results of the same experiment using a standard size of $1^{\circ}$ diameter (left) and $4^{\circ}$ diameter (right).

perceived to be of equal size as the standard. This pattern of results is present in all subjects.

\section{Effects of stimulus size}

The physiological data on receptive field shifts in area MT show an inverse relationship of the shift magnitude and the distance between attentional focus and original receptive field center (Womelsdorf et al., 2006). Assuming that the cue draws attention to the stimulus center, the distance of the attentional focus to the "critical" receptive fields overlapping the border of the stimulus depends on the stimulus size. The larger the stimulus, the less the critical receptive fields should shift and thus the smaller the effect of attention on size perception should be. In line with this argument, we performed the same experiment with two additional standard sizes. Figure $2 \mathrm{~B}$ shows the results from the experiments with $1^{\circ}$ standard diameter and 
$4^{\circ}$ standard diameter. In both experiments, the test cued curve is shifted to the left while the standard cued curve is shifted to the right, in line with the hypothesis that the allocation of attention onto the cued stimulus makes it appear larger. Consistent with the physiological data, this effect is negatively correlated with stimulus size: The average shift is $4 \%$ between neutral and cued trials for the larger standard size of $4^{\circ}(1 \% S D), 7 \%$ for the medium standard size of $2^{\circ}$, and $12 \%$ for the smaller standard size of $1^{\circ}(6 \% S D)$. The shift magnitudes were compared for the three different standard sizes using a $3 \times 3$ repeated measures ANOVA. The main effect of cue location on the PSE but not on the slope of the psychometric function (which represents the size discrimination threshold) is significant. Although there is no main effect of standard size on PSE, there is a significant interaction between standard size and cue location; that is, the difference in the magnitude of the PSE shift between the different standard sizes is significant. There is a main effect of standard size on the slope of the psychometric function, indicating that the size comparison is harder for smaller stimulus sizes. The interaction between cue location and standard size is not significant for the slope.

\section{Postcue control experiment}

The shifts of the PSE found in the main experiment are consistent with an increase of perceived size of the attended stimulus but could also result from a simple bias to select the cued over the uncued stimulus. To test for such a cue bias, we performed the postcue control experiment in which the order of cue and stimulus presentation was reversed: With the cue presented after the stimuli, a cue bias effect should remain while any attentional effect on the stimulus should disappear (see Gobell \& Carrasco, 2005). Figure 3 shows the average results of the postcue control experiment. As in the main experiment, the neutral cue condition yields a psychometric function with a PSE near 1 , but the psychometric curves in the test cued and standard cued conditions are

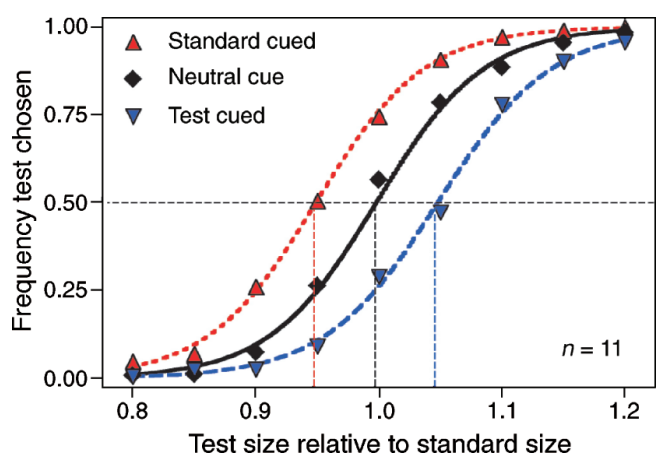

Figure 3. Average results with the cue presented after the RDPs. shifted in the opposite direction. The average shift of the PSE is $5 \%( \pm 2 \% S D)$. This means that while in the main experiment the cued stimulus is preferentially chosen as larger, in the postcue experiment the cued stimulus is chosen less frequently than the uncued stimulus. The $3 \times$ 2 repeated measures ANOVA comparing the main experiment with the postcue control experiment yields a significant main effect of cue location on the PSE but not on the slope and no main effect of cue presentation time on PSE or slope. The interaction between cue location and cue presentation time is significant for the PSE but not for the slope. Altogether, this indicates a shift of comparable magnitude but in opposite directions in the postcue and main experiments.

\section{Effects of instruction}

Although the results in the main experiment are not likely caused by a simple cue bias, we have not yet excluded a more complex kind of cue bias in which the cue has some influence on the stimulus that biases subjects to select it without increasing its apparent size. Therefore, we performed the reversed instructions control experiment, in which subjects had to evaluate the smaller stimulus: Here, an increase in perceived size would be reflected in a tendency to choose the cued stimulus less often than the uncued stimulus (see Carrasco et al., 2004; Fuller \& Carrasco, 2006; Montagna \& Carrasco, 2006; Turatto et al., 2007). Because an increase in perceived size and any cue bias would compensate each other in this design, the effect of attention might be underestimated or even occluded. A difference in the absolute size of the PSE shift between the main experiment and the reversed instructions experiment would indicate the presence of a bias effect.

We aimed at measuring the pure attentional effect in the single task control experiment, in which subjects had to indicate the smaller stimulus without reporting its motion direction. In this control experiment, there is no second task and therefore no cue bias related to it (see Carrasco et al., 2004). Therefore, although the magnitude of attentional modulation might be underestimated if subjects distribute their attention between the two stimuli, any effect that is observed reflects a pure attentional influence, providing a lower bound for the attentional effect magnitude. Figure 4 shows the average results of the reversed instructions control experiment and the single task control experiment. Because the $Y$-axis now plots the proportion of trials in which the test stimulus was chosen as smaller, the psychometric curves have an inverted shape. In both experiments, the PSE of the neutral cue condition matches the PPE and the test cued curve is shifted to the left while the standard cued curve is shifted to the right. This is consistent with the hypothesis that the cued stimulus is perceptually enlarged by attention and not consistent with a bias to select the cued stimulus more 


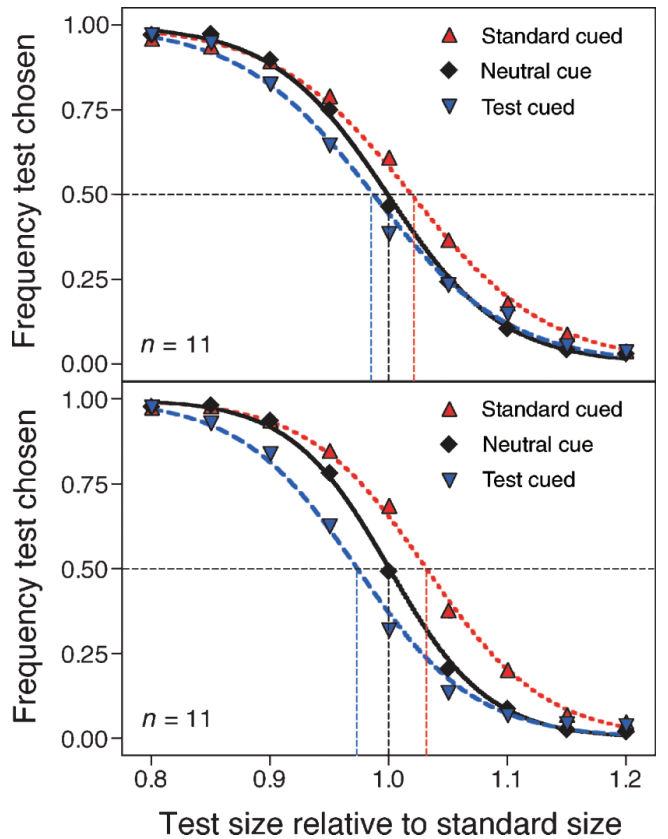

Figure 4. Average results of the reverse instructions and single task control experiments. Subjects were instructed to report the motion direction of the smaller stimulus (top) or to choose the smaller stimulus directly (bottom).

frequently. In comparison to the main experiment, average shift magnitudes are smaller in both control experiments (2\% $\pm 3 \% S D$ when the motion direction of the smaller stimulus had to be reported, $3 \% \pm 2 \% S D$ when the smaller stimulus had to be chosen directly without an evaluation of its motion direction). Two of the 11 subjects show a shift in the opposite direction when they had to report the motion direction of the smaller stimulus and no shift when the motion direction was not reported; the data from all other subjects qualitatively correspond to the average data. The main experiment and the two control experiments in which subjects had to choose the smaller stimulus were compared with a $3 \times 3$ repeated measures ANOVA. Again there is a main effect of cue location on PSE and size discrimination threshold (i.e., the absolute slopes of the psychometric function), and there is no main effect of instruction on PSE but on the slope. The interaction between cue location and instruction is significant for the PSE but not for the slope: The change in size perception is less when subjects choose the smaller instead of the larger stimulus.

\section{Direction discrimination performance}

To test for a facilitatory effect of attention in the second task, we compared the direction discrimination performance when subjects had chosen the cued or the uncued stimulus or when none of the stimuli were cued, disregarding if the choice of the larger or the smaller stimulus was correct or if test or standard stimulus was chosen. The $5 \times$ 3 repeated measures ANOVA (5 experiments $\times$ cued chosen/neutral/uncued chosen) shows a main effect of the chosen stimulus. Pairwise comparisons of the estimated marginal means reveal that subjects were better at evaluating the direction of motion when they evaluated the cued stimulus or when there was a neutral cue than when they evaluated the uncued stimulus (Figure 5, $p=.037$ and $p=.006$, respectively), although there is no performance difference between the "cued chosen" and the neutral trials. The performance in the direction discrimination task averaged over all cue conditions and all experiments is $75 \%( \pm 11 \% S D)$, indicating that subjects followed the instruction and paid attention to the direction task.

\section{Discussion}

In the main experiment, subjects reported the motion direction of the larger of two stimuli (test and standard), using a standard size of $2^{\circ}$ diameter. Attention on the test stimulus makes a smaller test stimulus appear equal to the standard stimulus, whereas attention on the standard stimulus makes a larger test stimulus appear equal to the standard stimulus. We found the same results with a standard size of $1^{\circ}$ and $4^{\circ}$ diameter, although the magnitude of the effect inversely varies with the size of

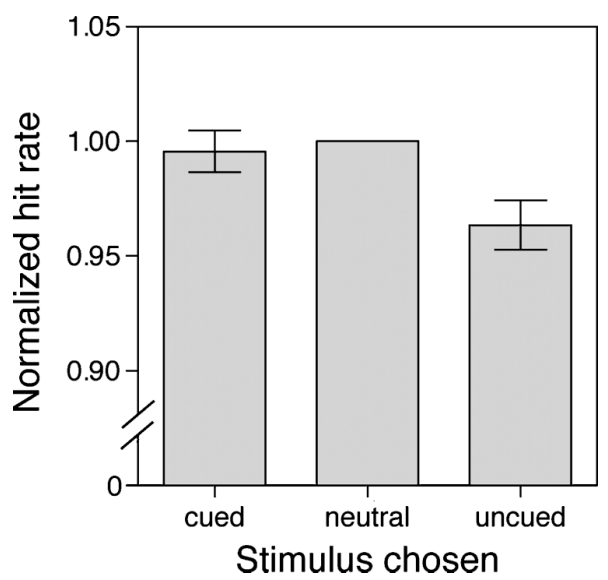

Figure 5. Direction discrimination performance. Average performance in the direction task independent of the size comparison task for trials in which the cued stimulus was evaluated, for neutral cue trials and for trials in which the uncued stimulus was evaluated. Hit rates were normalized to the neutral trial hit rate for each subject and then averaged. Error bars indicate the standard error of the mean. 


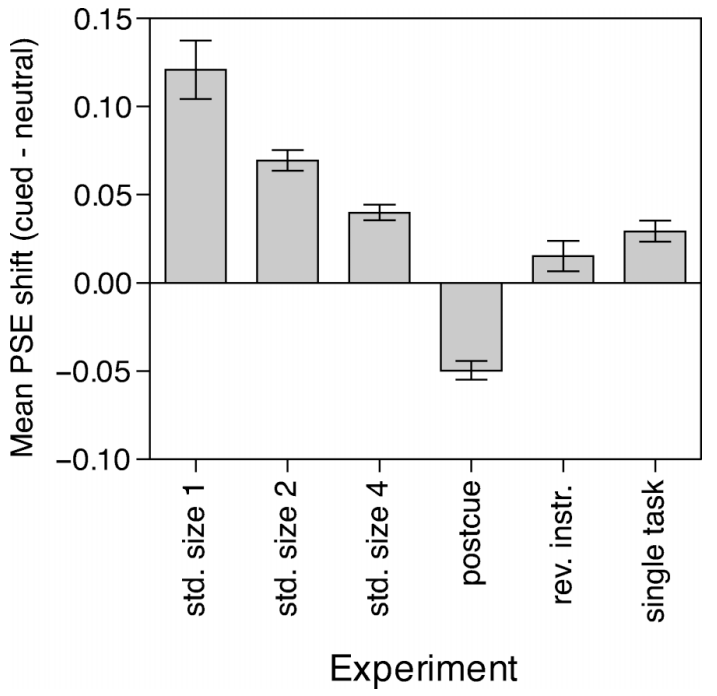

Figure 6. Overview of effect sizes. Shifts of the point of subjective equality (PSE) in cued trials relative to neutral trials were computed for each subject and then averaged across the 11 subjects. Error bars indicate the standard error of the mean.

the standard stimulus. Figure 6 summarizes the effect sizes for all experiments.

These PSE shifts show that subjects selected the cued stimulus more often than the uncued stimulus. This is in agreement with the hypothesis that the allocation of automatic attention to a stimulus increases its apparent size. Alternatively, a simple cue bias might lead subjects to respond to the cued stimulus preferentially. This kind of cue bias should persist when the cue is presented after the stimulus, whereas any attentional effect on stimulus selection should disappear. In the postcue experiment, there was no bias to choose the cued stimulus more often, supporting the interpretation of the PSE shifts as an attentional effect (Figure 6, postcue). Instead, the cued stimulus was chosen less frequently than the uncued stimulus. This might be explained with a masking effect: The postcue might mask the cued stimulus so that subjects tended to select the uncued stimulus simply because they were able to judge its motion direction more easily.

In the reverse instruction experiment, we tested whether attention has some other effect on the cued stimulus which causes a bias toward selecting it. Asking for the smaller instead of the larger stimulus allows to distinguish an effect on apparent size from a cue bias: A cue bias would still make subjects select the cued stimulus more often, whereas an attentional increase in perceived size of the cued stimulus would lead subjects to select it less often than the uncued stimulus. The observed PSE shift is consistent with an increase in perceived size by attention, although the effect is smaller than in the main experiment (Figure 6, reversed instructions). As indicated above, it is possible that two effects, a small cue bias and a large change in apparent size, partially compensate each other when subjects have to report the direction of the smaller RDP. The cue bias might result from a strategy of reporting the motion direction of the pattern which was easier to see: Attention might have a facilitatory effect and make direction discrimination easier for the cued pattern and so bias subjects to select it more often. In the main experiment, this "easiness" effect and an attentional increase in perceived size would add up, whereas in the reversed instructions experiment they act in opposite directions. The direction discrimination performance analysis shows that subjects are indeed better when they evaluate the cued than when they evaluate the uncued stimulus.

An effect of attentional facilitation of the direction discrimination is not expected in the single task experiment when subjects are asked to report the smaller stimulus without evaluating its direction of motion. Therefore, this experiment should measure the pure attentional modulation of perceived size, although the single task design likely underestimates the magnitude of attentional modulation: Because the single task asks for a direct comparison between the two stimuli, attention might be allocated more evenly between them. Consistent with this interpretation, we found a significant increase in perceived size that is intermediate between the effect observed in the main experiment and that in the reversed instruction experiment (Figure 6, single task).

Although we interpret our observations as a change in the perception of the stimulus, it should be noted that two studies have proposed alternative interpretations (Schneider, 2006, but see Ling \& Carrasco, in press; Turatto et al., 2007).

In several previous studies of attentional modulation of appearance, the effect was reduced when instructions were reversed meaning that subjects had to report on the stimulus containing the lesser quantity of a certain feature, for example, the stimulus of lower contrast, spatial frequency, saturation, and flicker (Carrasco et al., 2004; Fuller \& Carrasco, 2006; Gobell \& Carrasco, 2005; Montagna \& Carrasco, 2006; but for an exception, see Turatto et al., 2007). Subjects stated that it feels "more natural" for them to report the more of something than the less of something (Carrasco, personal communication). Thus, an inherent asymmetry favoring the selection of the stimulus containing the higher quantity of a certain feature might explain the smaller effect size with reversed instructions. This asymmetry might be an attentional asymmetry due to an automatic capture of attention by the stimulus of higher saliency.

Our study shows that when attention is drawn transiently to one of two stimuli, attention makes this stimulus appear larger. The magnitude of this effect correlates inversely with stimulus size. This is consistent with physiological data: Receptive field shift magnitudes in area MT vary inversely with the distance of the original receptive field center from the spatial focus of attention (Womelsdorf et al., 2006). If receptive field shifts are the 
physiological basis of attentional modulation of perceived size, the critical receptive fields for this effect would be those that overlap the stimulus borders. So the larger the stimulus, the larger is the distance between stimulus border and attentional focus, the smaller is the receptive field shift, and consequently the perceptual enlargement of the attended stimulus. Assuming that receptive field shifts are also a correlate of attentional facilitation of visual processing, this observation supports models of attention that assume a decay of attentional facilitation around the spatial focus of attention (Eriksen \& St. James, 1986). It is important to note though that this does not necessarily contradict models of attention that postulate a suppressive annulus around the facilitatory center of the attentional focus (Cutzu \& Tsotsos, 2003; Müller, Mollenhauer, Rösler, \& Kleinschmidt, 2005) in which the facilitatory effects of attention are reversed because the spatial extent of the facilitatory attentional focus and such a suppressive surround, given the particular settings employed in this experiment, is not tested. Because receptive field shifts with attention have not only been found in area MT but also in other cortical areas, the effect on perceived size reported here should not be restricted to moving stimuli.

Gobell and Carrasco (2005) have demonstrated that attention also modulates perceived spatial frequency and gap size in a Landolt stimulus. The shrinkage of receptive fields observed physiologically (Womelsdorf et al., 2006) could account for a shift of spatial frequency preferences to higher frequencies.

Gap size estimation depends on the precise localization of the end of the line segments forming the gap. If attention was drawn to the center of the Landolt stimulus, the gap should appear larger just like the overall stimulus. In Gobell and Carrasco's (2005) experiment, however, the cue was always on one side of the Landolt square, whereas the gap was either at the top or at the bottom. Therefore, although both line ends will be perceptually pushed away from the focus of attention, the magnitude could vary because their distance from the focus of attention differs, potentially creating an effect on perceived gap size.

Receptive field shifts might provide the physiological basis of the observed change in perceived stimulus size. By now a number of reports on the attentional modulation of the appearance of various stimulus parameters have been published though, which are not likely linked to spatial distortions of visual processing. Of all stimulus features so far tested, color (hue) seems to be the only one that is not perceived differently when attended. Hue is different from the other tested stimulus features: Although parameters like size, contrast, saturation, or motion coherence are varied quantitatively (prothetic scale), the variation of hue results in qualitatively different perceptual experiences (metathetic scale; see Fuller \& Carrasco, 2006; Stevens \& Galanter, 1957). From a functional perspective, attentional modulation of quantitative features like contrast or saturation could be beneficial because increasing those features could enhance discrimination of secondary features (it is easier to see the orientation of a high contrast pattern than that of a low contrast pattern), but there is no reason to assume that the variation of a qualitative feature would increase signal strength in the same way (judging the orientation of a purple stimulus should not be easier than judging the orientation of a blue stimulus). Following this line of thought, attentional modulation of appearance might not only be a side product of other attentional effects but might itself be functional for enhanced processing. The modulation of the appearance of many different quantitative but not qualitative features suggests that attention acts on a general level: Instead of modulating the appearance of several specific stimulus features via different unrelated physiological mechanisms, attention might generally increase the saliency of an attended stimulus and this enhanced saliency might then manifest itself in several distinct perceptual illusions.

\section{Conclusions}

In summary, we show that transient spatial attention increases the apparent size of moving visual stimuli. The increase in perceived size varies inversely with stimulus size, which is consistent with receptive field shift data (Womelsdorf et al., 2006). In addition, attention biases subjects to select the attended stimulus for perceptual judgment, possibly by decreasing the difficulty of the second task.

\section{Acknowledgments}

We thank Dr. Tzvetomir Tzvetanov for help with data analysis, Ralf Brockhausen for technical assistance, and Sabine Stuber for administrative assistance.

This work was supported by the International Max Planck Research School for Neurosciences, Goettingen, and the German Ministry for Education and Science Grant BMBF 01GQ0433 to the Bernstein Center for Computational Neuroscience, Goettingen.

Commercial relationships: none.

Corresponding author: Katharina Anton-Erxleben.

Email: kantonerxleben@dpz.gwdg.de.

Address: Cognitive Neuroscience Laboratory, German Primate Center, Kellnerweg 4, 37077 Goettingen, Germany.

References

Ben Hamed, S., Duhamel, J. R., Bremmer, F., \& Graf, W. (2002). Visual receptive field modulation in the 
lateral intraparietal area during attentive fixation and free gaze. Cerebral Cortex, 12, 234-245. [PubMed] [Article]

Bichot, N. P., Thompson, K. G., Chenchal Rao, S., \& Schall, J. D. (2001). Reliability of macaque frontal eye field neurons signalling saccade targets during visual search. Journal of Neuroscience, 21, 713-725. [PubMed] [Article]

Carrasco, M., Ling, S., \& Read, S. (2004). Attention alters appearance. Nature Neuroscience, 7, 308-313. [PubMed]

Connor, C. E., Gallant, J. L., Preddie, D. C., \& Van Essen, D. C. (1996). Responses in area V4 depend on the spatial relationship between stimulus and attention. Journal of Neurophysiology, 75, 1306-1308. [PubMed]

Connor, C. E., Preddie, D. C., Gallant, J. L., \& Van Essen, D. C. (1997). Spatial attention effects in macaque area V4. Journal of Neuroscience, 17, 3201-3214. [PubMed] [Article]

Cutzu, F., \& Tsotsos, J. K. (2003). The selective tuning model of attention: Psychophysical evidence for a suppressive annulus around an attended item. Vision Research, 43, 205-219. [PubMed]

Dobkins, K. R., \& Bosworth, R. G. (2001). Effects of setsize and selective spatial attention on motion processing. Vision Research, 41, 1501-1517. [PubMed]

Eriksen, C. W., \& St. James, J. D. (1986). Visual attention within and around the field of focal attention: A zoom lens model. Perception \& Psychophysics, 40, 225-240. [PubMed]

Fuller, S., \& Carrasco, M. (2006). Exogenous attention and color perception: Performance and appearance of saturation and hue. Vision Research, 46, 4032-4047. [PubMed]

Gobell, J., \& Carrasco, M. (2005). Attention alters the appearance of spatial frequency and gap size. Psychological Science, 16, 644-651. [PubMed]

Kusunoki, M., \& Goldberg, M. E. (2003). The time course of perisaccadic receptive field shifts in the lateral intraparietal area of the monkey. Journal of Neurophysiology, 89, 1519-1527. [PubMed] [Article]

Ling, S. \& Carrasco, M. (in press). Transient covert attention does alter appearance: A reply to Schneider (2006). Perception \& Psychophysics.

Liu, T., Fuller, S., \& Carrasco, M. (2006). Attention alters the appearance of motion coherence. Psychonomic Bulletin \& Review, 13, 1091-1096. [PubMed]
Mayfrank, L., Kimmig, H., \& Fischer, B. (1987). The role of attention in the preparation of visually guided saccadic movements in man. In J. K. O'Regan, \& A. LevySchoen (Eds.), Eye movements: From physiology to cognition (pp. 37-45). New York: North-Holland.

Montagna, B., \& Carrasco, M. (2006). Transient covert attention and the perceived rate of flicker. Journal of Vision, 6(9):8, 955-965, http://journalofvision.org/6/ 9/8/, doi:10.1167/6.9.8. [PubMed] [Article]

Müller, N. G., Mollenhauer, M., Rösler, A., \& Kleinschmidt, A. (2005). The attentional field has a Mexican hat distribution. Vision Research, 45, 1129-1137. [PubMed]

Posner, M. I. (1980). Orienting of attention. Quarterly Journal of Experimental Psychology, 32, 3-25. [PubMed]

Schneider, K. A. (2006). Does attention alter appearance? Perception \& Psychophysics, 68, 800-814. [PubMed]

Sperling, G. \& Dosher, B. A. (1986). Strategy and optimization in human information processing. In K. Boff, L. Kaufmann, \& J. P. Thomas (Eds.), Handbook of perception and performance (vol. 1). New York: Wiley.

Spitzer, H. \& Richmond, B. J. (1991). Task difficulty: Ignoring, attending to, and discriminating a visual stimulus yield progressively more activity in inferior temporal neurons. Experimental Brain Research, 83, 340-348. [PubMed]

Stevens, S. S., \& Galanter, E. H. (1957). Ratio scales and category scales for a dozen perceptual continua. Journal of Experimental Psychology, 54, 377-411. [PubMed]

Turatto, M., Vescovi, M., \& Valsecchi, M. (2007). Attention makes moving objects be perceived to move faster. Vision Research, 47, 166-178. [PubMed]

Urbach, D. \& Spitzer, H. (1995). Attentional effort modulated by task difficulty. Vision Research, 35, 2169-2177. [PubMed]

Wichmann, F. A., \& Hill, N. J. (2001). The psychometric function: I. Fitting, sampling, and goodness of fit. Perception \& Psychophysics, 63, 1293-1313. [PubMed] [Article]

Womelsdorf, T., Anton-Erxleben, K., Pieper, F., \& Treue, S. (2006). Dynamic shifts of visual receptive fields in cortical area MT by spatial attention. Nature Neuroscience, 9, 1156-1160. [PubMed]

Yeshurun, Y. \& Carrasco, M. (1998). Attention improves or impairs visual performance by enhancing spatial resolution. Nature, 396, 72-75. [PubMed] 



\section{Summary}

This thesis has investigated if attention modulates receptive field profiles (= spatial tuning curves) of single neurons in area MT, if the modulation of receptive field profiles also affects the inhibitory surround, and if such a change in spatial tuning can be explained by multiplicative modulation of feedforward input. Furthermore, we have hypothesized that shifts of receptive field profiles lead to perceptual distortions of spatial relations, specifically object sizes, and have tested if such distortions occur on the behavioral level.

The physiological part of this thesis shows that switching spatial attention between two stimuli presented within or near the classical receptive field of an MT neuron shifts the excitatory receptive field center as well as the inhibitory surround profile towards the attended stimulus. Shifts of the receptive field center are found even for receptive fields that are very far away from the attentional focus, in the opposite hemifield; for the inhibitory surround, such large distances were not tested. The shift of the inhibitory regions is on average larger in magnitude than the shift of the center, which means that the configuration of the receptive field center-surround structure is changed. Furthermore, attention changes the spatial summation characteristics of MT receptive fields depending on the distance between the attentional focus and the receptive field center by shrinking receptive fields around an attentional target inside the receptive field or expanding receptive fields towards an attentional target outside the receptive field; corresponding changes in baseline firing rate and suppression strength contribute to this effect. This concentrates more neuronal processing resources at the location of the attentional focus by bringing the attended stimulus into the most responsive regions of the receptive field. At the same time, suppression of influences from beyond the attentional focus is improved. The combination of these effects might serve to selectively strengthen the representation of the attended stimulus at the cost of unattended spatial locations and to enhance spatial resolution. In addition, the attentional effects on the inhibitory surround strength add to recent evidence that surround suppression may not be a fixed property of a neuron but is instead very 
flexible (Kapadia et al., 1999; Sceniak et al., 1999; Pack et al., 2005; Huang et al., 2007; Roberts et al., 2007).

Shifts of excitatory receptive field profiles have been observed earlier in the context of attention (Connor et al., 1996; Connor et al., 1997; Ben Hamed et al., 2002), and in the context of eye movements (Duhamel et al., 1991; Tolias et al., 2001), supporting the view that the planning of eye movements and attention share common mechanisms.

We provide evidence that both shrinkage and shift of the excitatory receptive field center can be accounted for by a feedforward model in which the gain of lowerlevel inputs, e.g. from V1, are differentially modulated in a multiplicative fashion. The computational study also enables us to estimate the spatial spread of the attentional focus. We suggest that a similar mechanism might underly a shift of the feedforward component of the receptive fields' inhibitory surround. Nevertheless, attentional modulation of receptive field profiles is effectively non-multiplicative at the level of MT neurons and therefore is an exception to the generally observed multiplication of tuning curves which does not change the selectivity of single neurons. This discrepancy might be related to the fact that spatial tuning is the only tuning that increases in width along the visual processing hierarchy: while direction selective cells, for example, sum inputs from lower level cells with similar preferences, spatially selective receptive fields sum lower level inputs tuned for different locations.

The psychophysical part of this thesis shows that attention increases the perceived size of moving visual stimuli. This study relates to several other recent studies demonstrating that attention can induce a change in the perceptual quality of a stimulus (Carrasco et al., 2004; Gobell and Carrasco, 2005; Liu et al., 2006; Fuller and Carrasco, 2006; Montagna and Carrasco, 2006; Turatto et al., 2007). We interprete the change of apparent size as a consequence of receptive field shifts: if the visual system uses a labeled-line principle to extract spatial position from the population activity of neurons with spatially restricted receptive fields, then shifting these receptive fields towards the center of the attended stimulus without updating their position label perceptually inflates the attended stimulus. 
The increase in perceived size varies inversely with stimulus size. This is consistent with a reduction of receptive field shifts for more distant targets, if one assumes that the critical receptive fields for the size illusion are those that overlap the borders of the stimulus, which are for larger stimuli further away from the attentional focus at the stimulus center. 



\section{Bibliography}

Albright TD, Desimone R, Gross, CG (1984) Columnar organization of directionally selective cells in visual area MT of the macaque. Journal of Neurophysiology 51(1): 16-31.

Allman J, Miezin F, McGuiness E (1985) Direction- and velocity-specific responses from beyond the classical receptive field in the middle temporal visual area (MT). Perception 14: 105-126.

Baizer JS, Ungerleider LG, Desimone R (1991) Organization of visual inputs to the inferior temporal and posterior parietal cortex in macaques. Journal of Neuroscience 11(1): 168-190.

Ben Hamed S, Duhamel JR, Bremmer F, Graf W (2002) Visual receptive field modulation in the lateral intraparietal area during attentive fixation and free gaze. Cerebral Cortex 12: 234-245.

Berezovskii VK, Born RT (2000) Specificity of projections from wide-field and local motion-processing regoins within the middle temporal visual area of the owl monkey. Journal of Neuroscience 20(3): 1157-1169.

Born RT, Bradley DC (2005) Structure and function of visual area MT. Annual Reviews Neuroscience 28: 157-189.

Born RT, Groh JM, Zhao R, Lukasewycz SJ (2000) Segregation of object and background motion in visual area MT: Effects of microstimulation on eye movements. Neuron 26(3): 725-734.

Born RT, Tootell RBH (1992) Segregation of global and local motion processing in primate middle temporal visual area. Nature 357: 497-499.

Bradley DC, Andersen RA (1998). Center-surround antagonism based on disparity in primate area MT. Journal of Neuroscience 18(18): 7552-7565.

Brefczynski JA, De Yoe EA (1999). A physiological correlate of the 'spotlight' of attention. Nature Neuroscience 2(4): 370-374.

Britten KH, Newsome WT, Shadlen MN, Celebrini S, Movshon JA (1996) A relationship between behavioral choice and the visual responses of neurons in macaque MT. Visual Neuroscience 13(1): 87-100.

Buracas GT, Albright TD (1996) Contribution of area MT to perception of threedimensional shape: a computational study. Vision Research 36(6): 869-887. 
Carrasco M, Penpeci-Talgar C, Eckstein M (2000) Spatial covert attention increases contrast sensitivity across the CSF: Support for signal enhancement. Vision Research 40(10-12): 1203-1215.

Carrasco M, Williams PE, Yeshurun Y (2002) Covert attention increases spatial resolution with or without masks: Support for signal enhancement. Journal of Vision 2: 467-479.

Carrasco M, Ling S, Read S (2004) Attention alters appearance. Nature Neuroscience 7(3): 308-313.

Churchland MM, Priebe NJ, Lisberger SG (2005) Comparison of the spatial limits on direction selectivity in visual areas MT and V1. Journal of Neurophysiology 93: 1235-1245.

Colby CL, Duhamel JR, Goldberg ME (1993) Ventral intraparietal area of the macaque: anatomic location and visual response properties. Journal of Neurophysiology 69(3): 902-914.

Connor CE, Gallant JL, Preddie DC, Van Essen DC (1996) Responses in area V4 depend on the spatial relationship between stimulus and attention. Journal of Neurophysiology 75: 1306-1309.

Connor CE, Preddie DC, Gallant JL, Van Essen DC (1997) Spatial attention effects in macaque area V4. Journal of Neuroscience 17(9): 3201-3214.

DeAngelis GC, Newsome WT (1999) Organization of disparity-selective neurons in macaque area MT. Journal of Neuroscience 19(4): 1398-1415.

Desimone R, Ungerleider LG (1986) Multiple visual areas in the caudal superior temporal sulcus of the macaque. Journal of Comparative Neurology 248: 164-189.

Dobkins KR, Bosworth RG (2001) Effects of set-size and selective spatial attention on motion processing. Vision Research 41: 1501-1517.

Dosher BA, Lu Z-L (2000) Mechanisms of perceptual attention in precuing of location. Vision Research 40: 1269-1292.

Dubner R, Zeki SM (1971) Response properties and receptive fields of cells in an anatomically defined region of the superior temporal sulcus in the monkey. Brain Research 35: 528-532.

Duhamel JR, Colby CL, Goldberg ME (1991) The updating of the representation of visual space in parietal cortex by intended eye movements. Science 255 : 90-92. 
Eckstein MP, Shimozaki SS, Abbey CK (2002) The footprints of visual attention in the Posner cueing paradigm revealed by classification images. Journal of Vision 2: 25-45.

Fries P, Reynolds JH, Rorie AE, Desimone R (2001) Modulation of oscillatory neuronal synchronization by selective visual attention. Science 291: 15601563.

Fuller S, Carrasco M (2006) Exogenous attention and color perception: Performance and appearance of saturation and hue. Vision Research 46: 4032-4047.

Gobell J, Carrasco M (2005) Attention alters the appearance of spatial frequency and gap size. Psychological Science16(8): 644-651.

Goodale MA, Milner AD (1992) Separate visual pathways for perception and action. Trends in Neurosciences 15(1): 20-25.

Graziano MSA, Andersen RA, Snowden RJ (1994) Tuning of MST neurons to spiral motions. Journal of Neuroscience 14(1): 54-67.

Haenny PE, Schiller PH (1988) State dependent activity in monkey visual cortex. I. Single cell activity in V1 and V4 on visual tasks. Experimental Brain Research 69(2): 225-244.

Hawkins HL, Hillyard SA, Luck SJ, Mouloua M, Downing C, Woodward DP (1990) Visual attention modulates signal detectability. Journal of Experimental Psychology: Human Perception and Performance 16(4): 802-811.

Huang X, Albright TD, Stoner GR (2007) Adaptive surround modulation in cortical area MT. Neuron 53: 761-770.

Hubel DH, Wiesel TN (1977) Ferrier lecture. Functional architecture of macaque monkey visual cortex. Proceedings of the Royal Society of London 198: 159.

Ito M, Gilbert CD (1999) Attention modulates contextual influences in the primary visual cortex of alert monkeys. Neuron 22: 593-604.

Joseph JS, Chun MM, Nakayama K (1997) Attentional requirements in a 'preattentive' feature search task. Nature 387: 805-807.

Kapadia MK, Westheimer G, \& Gilbert CD (1999) Dynamics of spatial summation in primary visual cortex of alert monkeys. Proceedings of the National Academy of Science USA 96(21): 12073-12078. 
Lennie P (1998) Single units and visual cortical organization. Perception 27(8): 889-935.

Lennie P (2003) Receptive fields. Current Biology 13(6): R216-R219.

Liu J, Newsome WT (2003) Functional organization of speed tuned neurons in visual area MT. Journal of Neurophysiology 89: 246-256.

Liu T, Fuller S, Carrasco M (2006) Attention alters the appearance of motion coherence. Psychonomic Bulletin \& Review 13: 1091-1096.

Liu T, Stevens ST, Carrasco M (2007) Comparing the time course and efficacy of spatial and feature-based attention. Vision Research 47: 108-113.

Liu L, Van Hulle MM (1998) Modeling the surround of MT cells and their selectivity for surface orientation in depth specified by motion. Neural Computation 10: 295-312.

Livingstone MS, Pack CC, Born RT (2001) Two-dimensional substructure of MT receptive fields. Neuron 30: 781-793.

Lui LL, Bourne JA, Rosa MGP (2007) Spatial summation, end inhibition and side inhibition in the middle temporal visual area (MT). Journal of Neurophysiology 97: 1135-1148.

Maunsell JHR, Van Essen DC (1983) The connections of the middle temporal visual area (MT) and their relationship to a cortical hierarchy in the macaque monkey. Journal of Neuroscience 3(12): 2563-2586.

Maunsell JHR, Van Essen DC (1983) Functional properties of neurons in middle temporal visual area of the macaque monkey. I. Selectivity for stimulus direction, speed, and orientation. Journal of Neurophysiology 49(5): 11271147.

Maunsell JHR, Van Essen DC (1987) Topographic organization of the middle temporal visual area in the macaque monkey: representational biases and the relationship to callosal connections and myeloarchitectonic boundaries. Journal of Comparative Neurology 266: 535-555.

Maunsell JHR, Nealey TA, DePriest DD (1990) Magnocellular and parvocellular contributions to reponses in the middle temporal visual area (MT) of the macaque monkey. Journal of Neuroscience 10(10): 3323-3334.

Martinez-Trujillo JC, Treue S (2002) Attentional modulation strength in cortical area MT depends on stimulus contrast. Neuron 35: 365-370. 
Martinez-Trujillo JC, Treue S (2004) Feature-based attention increases the selectivity of population responses in primate visual cortex. Current Biology 14: 744-751.

Martinez-Trujillo JC, Tsotsos JK, Simine E, Ponplun M, Wildes R, Treue S, Heinze HJ, Hopf JM (2005) Selectivity for speed gradients in human area MT / V5. Neuroreport 16(5): 435-438.

McAdams CJ, Maunsell JHR (1999) Effects of attention on orientation-tuning functions of single neurons in macaque cortical area V4. Journal of Neuroscience 19(1): 431-441.

Montagna B, Carrasco M (2006) Transient covert attention and the perceived rate of flicker. Journal of Vision 6(9): 955-965.

Moore T, Armstrong KM (2003) Selective gating of visual signals by microstimulation of frontal cortex. Nature 421: 370-373.

Moran J, Desimone R (1985) Selective attention gates visual processing in the extrastriate cortex. Science 229: 782-784.

Mounts JRW, Melara RD (1999) Attentional selection of objects or features: Evidence from a modified search task. Perception and Psychophysics 61(2): 322-341.

Movshon JA, Adelson EH, Gizzi MS, Newsome WT (1985). The analysis of moving visual patterns. In Chagas C, Gattass R, Gross C (eds.) Pattern recognition mechanisms - Pontificiae Academiae Scientiarum Scripta Varia 54. Heidelberg, Springer-Verlag: 117-151.

Movshon JA, Newsome WT (1996) Visual response properties of striate cortical neurons projecting to area MT in macaque monkeys. Journal of Neuroscience 16(23): 7733-7741.

Müller NG, Bartelt OA, Donner TH, Villringer A, Brandt SA (2003) A physiological correlate of the "zoom lens" of visual attention. Journal of Neuroscience 23(9): 3561-3565.

Müller NG, Kleinschmidt A (2003) Dynamic interaction of object- and space-based attention in retinotopic visual areas. Journal of Neuroscience 23(30): 98129816.

Nakayama K, Mackeben M (1989) Sustained and transient components of focal visual attention. Vision Research 29(11): 1631-1647. 
Nassi JJ, Callaway EM (2006) Multiple circuits relaying primate parallel visual pathways to the middle temporal area. Journal of Neuroscience 26(49): 12789-12798.

Newsome WT, Paré EB (1988) A selective impairment of motion perception following lesions of the middle temporal visual area (MT). Journal of Neuroscience 8(6): 2201-2211.

Niebur E, Hsiao S, Johnson KO (2002) Synchrony: a neuronal mechanism for attentional selection? Current Opinion in Neurobiology 12: 190-194.

O'Connor DH, Fukui MM, Pinsk MA, Kastner S (2002) Attention modulates responses in the human lateral geniculate nucleus. Nature Neuroscience 5(11): 1203-1209.

O'Craven KM, Downing PE, Kanwisher N (1999) FMRI evidence for objects as the units of attentional selection. Nature 401: 584-587.

Olshausen BA, Anderson CH, Van Essen DC (1993) A neurobiological model of visual attention and invariant pattern recognition based on dynamic routing of information. Journal of Neuroscience 13(11): 4700-4719.

Orban GA, Kennedy H, Bullier J (1986) Velocity sensitivity of neurons in areas V1 and V2 of the monkey: influence of eccentricity. Journal of Neurophysiology 56(2): 462-480.

Pack CC, Conway BR, Born RT, Livingstone MS (2006) Spatiotemporal structure of nonlinear subunits in macaque visual cortex. Journal of Neuroscience 26(3): 893-907.

Pack CC, Hunter JN, Born RT (2005) Contrast dependence of suppressive influences in cortical area MT of alert macaque. Journal of Neurophysiology 93: 1809-1815.

Perge JA, Borghuis BG, Bours RJE, Lankheet MJM, van Wezel RJA (2005) Dynamics of directional selectivity in MT receptive field centre and surround. European Journal of Neuroscience 22: 2049-2058.

Posner, MI (1980) Orienting of attention. Quarterly Journal of Experimental Psychology 32: 3-25.

Raiguel S, Van Hulle MM, Xiao DK, Marcar VL, Orban GA (1995) Shape and spatial distribution of receptive fields and antagonistic motion surrounds in the middle temporal area (V5) of the macaque. European Journal of Neuroscience 7(10): 2064-2083. 
Regan D (1986) Visual processing of four kinds of relative motion. Vision Research 26(1): 127-145.

Reynolds JH, Chelazzi L, Desimone R (1999) Competitive mechanisms subserve attention in macaque areas V2 and V4. Journal of Neuroscience 19(5): 1736-1753.

Reynolds JH, Pasternak T, Desimone R (2000) Attention increases sensitivity of V4 neurons. Neuron 26: 703-714.

Rizzolatti G, Riggio L, Dascola I, Umilta C (1987 ) Reorienting attention across the horizontal and vertical meridians: evidence in favor of a premotor theory of attention. Neuropsychologia 25(1A): 31-40.

Roberts M, Delicato LS, Herrero J, Gieselmann MA, Thiele A (2007) Attention alters spatial integration in macaque $\mathrm{V} 1$ in an eccentricity-dependent manner. Nature Neuroscience 10(11): 1483-1491.

Roelfsema PR, Lamme VAF, Spekreijse H (1998) Object-based attention in the primary visual cortex of the macaque monkey. Nature 395: 376-381.

Rust NC, Mante V, Simoncelli EP, Movshon JA (2006) How MT cells analyze the motion of visual patterns. Nature Neuroscience 9(11): 1421-1431.

Sàenz M, Buracas G, Boynton GM (2002) Global effects of feature-based attention in human visual cortex. Nature Neuroscience 5(7): 631-632.

Sàenz M, Buracas G, Boynton GM (2003) Global feature-based attention for motion and color. Vision Research 43: 629-637.

Salinas E, Abbott LF (1997) Invariant visual responses from attentional gain fields. Journal of Neurophysiology 77(6): 3267-3272.

Salinas E, Thier P (2000) Gain modulation: a major computational principle of the central nervous system. Neuron 27: 15-21.

Salzmann CD, Britten KH, Newsome WT (1990) Cortical microstimulation influences perceptual judgements of motion direction. Nature 346: 174-177.

Sceniak MP, Ringach DL, Hawken MJ, Shapley R (1999) Contrast's effect on spatial summation by macaque V1 neurons. Nature Neuroscience 2(8): 733-739.

Serences JT, Schwarzbach J, Courtney SM, Golay X, Yantis S (2004) Control of object-based attention in human cortex. Cerebral Cortex 14: 1346-1357.

Serences JT, Yantis S (2006) Selective visual attention and perceptual coherence. Trends in Cognitive Sciences 10(1): 38-45. 
Sincich LC, Horton JC (2003) Independent projection streams from macaque striate cortex to the second visual area and middle temporal area. Journal of Neuroscience 23(13): 5684-5692.

Sincich LC, Park KF, Wohlgemuth MJ, Horton JC (2004) Bypassing V1: a direct geniculate input to area MT. Nature Neuroscience 7(10): 1123-1128.

Snowden RJ, Treue S, Erickson RG, Andersen RA (1991) The response of area MT and V1 neurons to transparent motion. Journal of Neuroscience 11(9): 2768-2785.

Spitzer H, Desimone R, Moran J (1988) Increased attention enhances both behavioral and neuronal performance. Science 240(4850): 338-340.

Steinmetz PN, Roy A, Fitzgerald PJ, Hsiao SS, Johnson KO, Niebur E (2000) Attention modulates synchronized neuronal firing in primate somatosensory cortex. Nature 404: 187-190.

Tadin D, Lappin JS, Gilroy LA, Blake R (2003) Perceptual consequences of centre-surround antagonism in visual motion processing. Nature 424: 312315.

Tanaka K, Hikosaka K, Saito H, Yukie M, Fukada Y, Iwai E (1986) Analysis of local and wide-field movements in the superior temporal visual areas of the macaque monkey. Journal of Neuroscience 6(1): 134-144.

Tolias AS, Moore T, Smirnakis SM, Tehovik EJ, Siapas AG, Schiller PH (2001) Eye movements modulate visual receptive fields of V4 neurons. Neuron 29: 757-767.

Tootell RBH, Hadjikhani N, Hall EK, Marrett S, Vanduffel W, Vaughan JT, Dale AM (1998) The retinotopy of visual spatial attention. Neuron 21: 1409-1422.

Treisman A, Gelade G (1980) A feature-integration theory of attention. Cognitive Psychology 12: 97-136.

Treue S (2003) Climbing the cortical ladder from sensation to perception. Trends in Cognitive Sciences 7(11): 469-471.

Treue S (2003) Visual attention: the where, what, how and why of saliency. Current Opinion in Neurobiology 13: 428-432.

Treue S, Husain M, Andersen RA (1991) Human perception of structure from motion. Vision Research 31(1): 59-75.

Treue S, Andersen RA (1996) Neural responses to velocity gradients in macaque cortical area MT. Visual Neuroscience 13: 797-804. 
Treue S, Maunsell JHR (1996) Attentional modulation of visual motion processing in cortical areas MT and MST. Nature 382: 539-541.

Treue S, Martinez-Trujillo JC (1999) Feature-based attention influences motion processing gain in macaque visual cortex. Nature 399: 575-579.

Treue S, Maunsell JHR (1999) Effects of attention on the processing of motion in macaque middle temporal and medial superior temporal visual cortical areas. Journal of Neuroscience 19(17): 7591-7602.

Turatto M, Vescovi M, Valsecchi M (2007) Attention makes moving objects be perceived to move faster. Vision Research 47: 166-178.

Ungerleider LG, Desimone R (1986) Cortical connections of visual area MT in the macaque. Journal of Comparative Neurology 248: 190-222.

Ungerleider LG, Mishkin M (1982) Two cortical systems. In Ingle DJ, Goodale MA, Mansfield RJW (eds) Analysis of Visual Behavior. Cambridge, MIT Press: 549-586.

Warren WH, Hannon DJ (1988) Direction of self-motion is perceived from optical flow. Nature 336: 162-163.

Williford T, Maunsell JHR (2004) Effects of spatial attention on contrast response functions in macaque area V4. Journal of Neurophysiology 96: 40-54.

Wojciulik E, Kanwisher N, Driver J (1998) Covert visual attention modulates facespecific activity in the human fusiform gyrus: fMRI study. Journal of Neurophysiology 79(3): 1574-1578.

Xiao DK, Raiguel S, Marcar V, Koenderink J, Orban GA (1995) Spatial heterogeneity of inhibitory surrounds in the middle temporal visual area. Proceedings of the National Academy of Science USA 92: 11303-11306.

Yabuta NH, Sawatari A, Callaway EM (2001) Two functional channels from primary visual cortex to dorsal visual cortical areas. Science 292: 297-300.

Yeshurun Y, Carrasco M (1998) Attention improves or impairs visual performance by enhancing spatial resolution. Nature 396: 72-75.

Yeshurun Y, Carrasco M (1999) Spatial attention improves performance in spatial resolution tasks. Vision Research 39: 293-306.

Zeki, SM (1974) Functional organization of a visual area in the posterior bank of the superior temporal sulcus of the rhesus monkey. Journal of Physiology 236: 549-573. 
Zihl J, von Cramon D, Mai N (1983) Selective disturbance of movement vision after bilateral brain damage. Brain 106: 313-340. 


\title{
Curriculum Vitae
}

\author{
KATHARINA ANTON-ERXLEBEN
}

\section{Personal details}

Date of birth 25th of March, 1980

Place of birth Stanford, USA

\section{Scientific education}

since 2004

$2002-2004$

$2001-2002$

$1999-2001$
Graduate student, German Primate Center, Göttingen, Germany

Advisor: Prof. Dr. Stefan Treue

M. Sc. Neurosciences, Graduate Program of the International Max Planck Research School for Neuroscience, Göttingen, Germany

Thesis: Psychophysics and functional brain imaging of attentional modulation, German Primate Center

Advisors: Prof. Dr. Stefan Treue and Prof. Dr. Jens Frahm

Undergraduate studies in psychology, Freie Universität Berlin, Germany

Undergraduate studies in psychology, Universität Konstanz, Germany

Abitur, Kieler Gelehrtenschule, Kiel, Germany

\section{Laboratory experience}

several lab rotation projects in the Graduate Program Neurosciences:

- Attentional modulation of receptive field structure within motion sensitive area MT - an extracellular recording approach, German Primate Center Advisors: Prof. Dr. Stefan Treue, Thilo Womelsdorf

- Functional neuroimaging of humans: Retinotopic organization of human V1, MPI for biophysical chemistry

Advisor: Prof. Dr. Jens Frahm 
- Experience-dependent synaptic plasticity in the Drosophila neuromuscular junction, European Neuroscience Institute

Advisors: Dr. Stefan Sigrist, Tobias Rasse

- Regulation of TGF- $\beta$ in the developing chick retina, Universität Göttingen

Advisors: Dr. Nicole Dünker, Dr. Astrid Baur

2002

Research assistant, Technische Universität Berlin

Project: Representation of time-related text information

Advisor: Prof. Dr. Stephanie Kelter

\section{Teaching experience}

$2006-2007$

$2004-2007$

$2004-2006$

2001
Supervision of a diploma thesis project on the physiology of attentional modulation, German Primate Center, Göttingen

Several tutorials on visual perception and attention for undergraduate students in the Graduate Program Neurosciences, Göttingen

Supervision of several lab rotation projects in the Graduate Program Neurosciences, German Primate Center, Göttingen

Weekly tutorial for undergraduate students in biological psychology, Universität Konstanz, Germany

\section{Courses \& workshops}

October 2005

September 2005

September 2004
Workshop "Analysis and Models in Neurophysiology" in Freiburg organized by the German Society for Neuroscience (NWG)

Computational Neuroscience Course in Göttingen, organized by the Bernstein Center for Computational Neuroscience (BCCN) and the German Society for Neuroscience (NWG)

Summer School Visual Neuroscience in Rauischholzhausen, organized by the Universities of Giessen und Marburg

Poster: Attentional modulation of neural activity in different stages of the visual hierarchy 


\section{Conference contributions}

November 2007

September 2007

March 2007

October 2006
Society for Neuroscience (SFN) Annual Meeting in San Diego, USA

Poster: Attention modulates receptive field structure in macaque area MT

3rd Bernstein Symposium of the Bernstein Center for Computational Neuroscience in Göttingen, Germany

Presentation: Attention modulates receptive field structure in macaque area MT

Göttinger Neurobiologentagung, Göttingen, Germany

Poster: Attention changes perceived size of moving visual patterns

Society for Neuroscience (SFN) Annual Meeting in Atlanta, USA

Poster: Attention changes perceived size of moving visual patterns

\section{Publications}

Prepared for submission

In revision
Attention modulates center-surround structure in area MT Anton-Erxleben K, Stephan VM, Treue S

Receptive field shift and shrinkage in macaque area MT through attentional gain modulation

Womelsdorf T, Tzvetanov T, Anton-Erxleben K, Treue S, Journal of Neuroscience

Attention changes perceived size of moving visual patterns

Anton-Erxleben K, Henrich C, Treue S, Journal of Vision 7(11): 1-9

2006
Dynamic shifts of visual receptive fields in cortical area MT by spatial attention

Womelsdorf T, Anton-Erxleben K, Pieper F, Treue S, Nature Neuroscience 9(9): 1156-1160 